\title{
Analysis of Multilevel Finite Volume Approximation of 2D Convective Cahn-Hilliard Equation
}

\author{
A. R. Appadu ${ }^{a}$, J. K. Djoko ${ }^{a}$ 1, H. H. Gidey ${ }^{a, b}$ and J. M. S. Lubuma ${ }^{a}$ \\ ${ }^{a}$ Department of Mathematics and Applied Mathematics, University of Pretoria, Pretoria, 0002, South \\ Africa. \\ ${ }^{b}$ Department of Computational Sciences, Aksum University, Aksum, 1010, Ethiopia.
}

\begin{abstract}
In this work, four finite volume methods have been constructed to solve the 2D convective CahnHilliard equation with specified initial condition and periodic boundary conditions. We prove existence and uniqueness of solutions. The stability and convergence analysis of the numerical methods have been discussed thoroughly. The nonlinear terms are approximated by a linear expression based on Mickens' rule [1 of nonlocal approximations of nonlinear terms. Numerical experiments for a test problem have been carried out to test all methods.
\end{abstract}

Keywords: 2D convective Cahn-Hilliard equation, existence of solution, uniqueness, stability, convergence, finite volume, multilevel

\section{Introduction}

The general setting of this work is the $2 \mathrm{D}$ convective Cahn-Hilliard equation:

$$
u_{t}-\gamma u(\beta \cdot \nabla u)+\varepsilon^{2} \Delta^{2} u=\Delta f(u),(x, y) \in \mathcal{M}, t>0,
$$

with initial condition

$$
u(x, y, 0)=u^{0}(x, y), \quad(x, y) \in \overline{\mathcal{M}},
$$

and periodic boundary conditions

$$
\begin{aligned}
& \frac{\partial^{j} u}{\partial x^{j}}\left(-L_{1}, y, t\right)=\frac{\partial^{j} u}{\partial x^{j}}\left(L_{1}, y, t\right), \quad y \in\left(-L_{2}, L_{2}\right) \text { and } 0 \leq t \leq T, \\
& \frac{\partial^{j} u}{\partial y^{j}}\left(x,-L_{2}, t\right)=\frac{\partial^{j} u}{\partial y^{j}}\left(x, L_{2}, t\right), \quad x \in\left(-L_{1}, L_{1}\right) \text { and } 0 \leq t \leq T,
\end{aligned}
$$

where

$$
f(u)=u^{3}-u,
$$

$\gamma$ is the driving force, $j=0,1,2,3, \mathcal{M}=\left(-L_{1}, L_{1}\right) \times\left(-L_{2}, L_{2}\right), \overline{\mathcal{M}}$ is the closure of $\mathcal{M}, L_{1}$ and $L_{2}$ are positive constants, $u^{0} \in L^{2}(\mathcal{M}), \varepsilon$ is a dimensionless interfacial width and $\beta$ is a vector in 2D.

This equation is a successful model for the description of several physical phenomena: spinodal decomposition of phase separating systems in the presence of an external field (e.g. gravitational, magnetic and electronic) [2, 3, 4, formation of facets and corners in crystal growth [5, 6].

In the absence of the driving field, i.e. $\gamma=0$, the system reduced to the well known Cahn-Hilliard equation

$$
u_{t}+\varepsilon^{2} \Delta^{2} u=\Delta f(u)
$$

\footnotetext{
${ }^{1}$ Corresponding author: Jules.Djokokamdem@up.ac.za
} 
which is a model to describe the evolution of a concentration field for a binary mixture [7] and phase separation of binary liquids or binary alloys [8]. This reduced model has been studied by several authors (see 19, 10, 11] and the references therein). In [11, higher order schemes preserving the properties such as energy and large time behavior are constructed. The Cahn-Hilliard equation, (1.5), admits a Lyapunov (free energy) functional which guarantees that generically all solutions converge to an equilibrium.

The one-dimensional case of 1.1 has been studied by several researchers, theoretically and numerically. Analytical solutions have been obtained for a single interface in the presence of the driving force, i.e. $\gamma \neq 0$, in an infinite system [2]. The effect of this driving force on the coarsening dynamics of the one-dimensional Cahn-Hilliard equation at $T=0$ has been studied by Emmott and Bray [3] when $\varepsilon=1$. They observed that the driving force $\gamma$ has an asymmetric effect on the solution of a single stationary domain wall. They also noted that the behavior of the kink-anti kink pair (bubble) depends on $\gamma^{-1}$ and the separation of the interfaces. Later, Golovin et al. [12] demonstrated numerically that the one-dimensional convective CahnHilliard equation exhibits a transition from coarsening to chaotic behaviour as $\gamma$ increases. The presence of the driving force elucidates a fundamental asymmetry between kinks and anti-kinks which is not present in the Cahn-Hilliard theory [13. In Podolny et al. [14, the dynamics of domain walls (kinks) governed by the convective Cahn-Hilliard equation is studied by means of asymptotic and numerical methods. The bifurcations of stationary solutions for different values of $\gamma$ with $\varepsilon=1$ has been studied by Zaks et. al [15. Eden and Kalantarov [16] proved the existence of compact attractor and a finite inertial manifold that contains it and Zhao and Liu [17] proved the existence of optimal solutions for the one dimensional convective Cahn-Hilliard Equation. Aderogba et al. [18] solved the one dimensional convective Cahn-Hilliard equation numerically using fractional step-splitting methods for $\gamma=0.1$ and $\varepsilon=1$. The authors observe that the solution coarsens as $t$ progresses and they tested numerically the transition of convective CahnHilliard equation from coarsening to an order less pattern as $\gamma$ increases, which is the behavior of Kuramoto Sivashinsky equation.

For multi-dimensional convective Cahn-Hilliard equation, the transition to roughening and the structure of the steady states are not well understood [3, 12, 19]. The existence of optimal solutions for the 2D convective Cahn-Hilliard equation has been proved by Zhao and Liu 20. Eden and Kalantarov [19] considered the 3D convective Cahn-Hilliard equation with periodic boundary conditions and proved the existence of absorbing balls.

It is worth noting that (1.1) together with 1.3 and 1.4 , for $j=0$, leads to

$$
\iint_{\mathcal{M}} u(x, y, t) d x d y=\iint_{\mathcal{M}} u^{0}(x, y) d x d y, \quad \forall t
$$

Hence for the analysis of 1.1]-1.4, it is important to assume that 21]

$$
\iint_{\mathcal{M}} u^{0}(x, y) d x d y=0
$$

Our objective is to propose numerical techniques based on the work in [22, 23] to compute the numerical solution of 1.1-1.4.

In this work, we focus on the numerical solution of the 2D convective Cahn-Hilliard equation with $\gamma=1$ and $\beta=\langle 1,1\rangle$, using multilevel finite volume methods. Multilevel methods were introduced to improve calculation speed in the simulation of complex physical phenomena while maintaining an accurate solution 22, 23, 24, 25, 26, 27. We construct two schemes associated with (1.1)-(1.4) based on the work of Bousquet et al. 23]. The schemes we construct are easy to implement and are respectively called:

(a) linear implicit multilevel approximation, and

(b) explicit multilevel approximation. 
Our contribution can be regarded as extension to the works of Bousquet et al. 22, 23. Indeed, in the latter $1 \mathrm{D}$ advection equation is analyzed and 2D shallow water linearized around a constant flow is proposed and implemented. In contrast, in our work we tackle fourth order $2 \mathrm{D}$ nonlinear partial differential equation. One of the challenge as mentioned earlier is to discretize the nonlinear term $u(\beta \cdot \nabla u)$ in a linear way while maintaining basic properties, and as a consequence saving computational time.

For the sake of comparison, we also formulate two one-level methods associated to the multilevel methods. One of the difficulties is to design an appropriate linear expression for the nonlinear term. We achieve that thanks to the nonlocal approximation of nonlinear quantity introduced by Mickens [1] and Anguelov [28]. In particular, following [29, we approximate the nonlinear term $u(\beta \cdot \nabla u)$ in a linear way such that the property

$$
\iint_{\mathcal{M}} u(\boldsymbol{\beta} \cdot \nabla u) u d x d y=0
$$

is constructed at the discrete level.

After the construction of new schemes, we show the existence and uniqueness of the solution. At this step, we should bear in mind that since we are dealing with linear equations in finite dimension, existence of solutions is equivalent to uniqueness, thus, we provide conditions under which there is one solution. Of course, this analysis is only done for the one-level implicit scheme and easily extended to multilevel. The third contribution of this work is the stability of the new schemes. We show that the implicit multilevel method is conditionally stable with a region of stability smaller than one obtained from the one-level implicit method on the fine mesh. The fourth contribution of our study is the convergence analysis of the implicit methods. Indeed, we show that the implicit methods are first order accurate in time and second order accurate in space. Our last contribution is numerical result that supports our theoretical findings. We compute $L_{2}$-error and rate of convergence for the proposed numerical methods. We also demonstrate that in all numerical tests, the multilevel methods are faster than the one-level methods on the fine mesh.

The rest of this work is organized as follows: in the next section, we recall some preliminaries and introduce some standard notations. We also discuss, in Section 2, some properties of difference operators and the discrete analogue of $L_{2}$ space. In Sections 3 and 4, we construct one-level and multilevel finite volume methods and proved those methods are conditionally stable and conditionally convergent. In Section 5 , we present some numerical results comparing computations done by one-level methods and computations done by the multilevel methods. Lastly, conclusions are given in Section 6 .

\section{Some Preliminaries and Space Discretizations}

In this section, we recall some preliminaries which are helpful to our discussion and we present the space discretization in a $2 \mathrm{D}$ rectangular region. To develop finite volume approximations that satisfy the discrete analogue of (1.7), we first introduce some standard notations and results. We partition $\mathcal{M}$ into $N_{1} \times N_{2}$ control volumes $\left(k_{i, j}\right)_{1 \leq i \leq N_{1}, 1 \leq j \leq N_{2}}$ of uniform area $\Delta x \Delta y$, where $\Delta x$ and $\Delta y$ are the spatial step sizes in the $x$ - and $y$ - directions, respectively. It is assumed that the partition of the domain is conform, meaning that for two elements $A$ and $B$ one has, $A \cap B$ is either a face, a vertex or empty set. For $0 \leq i \leq N_{1}$ and $0 \leq j \leq N_{2}$,

$$
x_{i+1 / 2}=i \Delta x-L_{1}, y_{j+1 / 2}=j \Delta y-L_{2},
$$

so that

$$
k_{i, j}=\left(x_{i-1 / 2}, x_{i+1 / 2}\right) \times\left(y_{j-1 / 2}, y_{j+1 / 2}\right) \text { for } 1 \leq i \leq N_{1}, 1 \leq j \leq N_{2} .
$$

$\left(x_{i}, y_{j}\right)$ is the centre of the $(i, j)$ control volume, which is given by the formula

$$
\left(x_{i}, y_{j}\right)=\left((i-1) \Delta x+\frac{\Delta x}{2}-L_{1},(j-1) \Delta y+\frac{\Delta y}{2}-L_{2}\right), \quad 1 \leq i \leq N_{1}, 1 \leq j \leq N_{2} .
$$


In the rest of this work, we take $h=(\Delta x, \Delta y)$. The approximate solution to the control volume average of the true solution at $t_{n}=n \Delta t$ is denoted by $u_{i, j}^{n}$, i.e.

$$
u_{i, j}^{n} \approx \frac{1}{\Delta x \Delta y} \iint_{k_{i, j}} u\left(x, y, t_{n}\right) d x d y, 1 \leq i \leq N_{1}, 1 \leq j \leq N_{2},
$$

where $\Delta t$ is the temporal step size such that $\Delta t M=T$, which is obtained recursively by starting with the initial average value, $u_{i, j}^{0}$, given by

$$
u_{i, j}^{0}=\frac{1}{\Delta x \Delta y} \iint_{k_{i, j}} u^{0}(x, y) d x d y, 1 \leq i \leq N_{1}, 1 \leq j \leq N_{2} .
$$

Define the space $\mathcal{H}_{h}$ as

$$
\mathcal{H}_{h}=\left\{\mathbf{u}=\left(u_{i, j}\right)_{i, j \in \mathbb{Z}}, u_{i, j} \in \mathbb{R} \mid u_{i+N_{1}, j}=u_{i, j}=u_{i, j+N_{2}}, \text { and } \sum_{i=1}^{N_{1}} \sum_{j=1}^{N_{2}} u_{i, j}=0\right\},
$$

equipped with the inner product and discrete $L^{2}$ norm

$$
(\mathbf{u}, \mathbf{v})_{h}=\Delta x \Delta y \sum_{i=1}^{N_{1}} \sum_{j=1}^{N_{2}} u_{i, j} v_{i, j} \text { and }\|\mathbf{u}\|_{h}=\left(\Delta x \Delta y \sum_{i=1}^{N_{1}} \sum_{j=1}^{N_{2}} u_{i, j}^{2}\right)^{1 / 2},
$$

respectively.

For $\mathbf{u} \in \mathcal{H}_{h}$, we introduce the following difference operators:

$$
\begin{aligned}
\nabla_{1, h}^{-} u_{i, j} & =\frac{1}{\Delta x}\left(u_{i, j}-u_{i-1, j}\right), \nabla_{1, h}^{+} u_{i, j}=\frac{1}{\Delta x}\left(u_{i+1, j}-u_{i, j}\right), \\
\nabla_{2, h}^{-} u_{i, j} & =\frac{1}{\Delta y}\left(u_{i, j}-u_{i, j-1}\right), \nabla_{2, h}^{+} u_{i, j}=\frac{1}{\Delta y}\left(u_{i, j+1}-u_{i, j}\right), \\
\Delta_{1, h} u_{i, j} & =\frac{1}{\Delta x^{2}}\left(u_{i+1, j}-2 u_{i, j}+u_{i-1, j}\right) \\
\Delta_{2, h} u_{i, j} & =\frac{1}{\Delta y^{2}}\left(u_{i, j+1}-2 u_{i, j}+u_{i, j-1}\right) \\
\Delta_{1, h}^{2} u_{i, j} & =\frac{1}{\Delta x^{2}}\left(\Delta_{1, h} u_{i+1, j}-2 \Delta_{1, h} u_{i, j}+\Delta_{1, h} u_{i-1, j}\right) \\
\Delta_{2, h}^{2} u_{i, j} & =\frac{1}{\Delta y^{2}}\left(\Delta_{2, h} u_{i, j+1}-2 \Delta_{2, h} u_{i, j}+\Delta_{2, h} u_{i, j-1}\right) .
\end{aligned}
$$

From (2.1)-2.6), we have

$$
\beta \cdot \nabla_{h}^{ \pm}=\nabla_{1, h}^{ \pm}+\nabla_{2, h}^{ \pm}, \Delta_{h}=\Delta_{1, h}+\Delta_{2, h}, \Delta_{h}^{2}=\Delta_{1, h}^{2}+\Delta_{1, h} \Delta_{2, h}+\Delta_{2, h} \Delta_{1, h}+\Delta_{2, h}^{2} .
$$

The discrete analogue of the derivative of product of functions is given as follows: for $\mathbf{u}, \mathbf{v} \in \mathcal{H}_{h}$,

$$
\begin{aligned}
& \left(\boldsymbol{\beta} \cdot \nabla_{h}^{+}\right)\left(u_{i, j} v_{i, j}\right)=\left(\nabla_{1, h}^{+} u_{i, j}\right) v_{i+1, j}+u_{i, j}\left(\nabla_{1, h}^{+} v_{i, j}\right)+\left(\nabla_{2, h}^{+} u_{i, j}\right) v_{i, j+1}+u_{i, j}\left(\nabla_{2, h}^{+} v_{i, j}\right), \\
& \left(\boldsymbol{\beta} \cdot \nabla_{h}^{-}\right)\left(u_{i, j} v_{i, j}\right)=\left(\nabla_{1, h}^{-} u_{i, j}\right) v_{i-1, j}+u_{i, j}\left(\nabla_{1, h}^{-} v_{i, j}\right)+\left(\nabla_{2, h}^{-} u_{i, j}\right) v_{i, j-1}+u_{i, j}\left(\nabla_{2, h}^{-} v_{i, j}\right) .
\end{aligned}
$$

From the definition of $\mathcal{H}_{h}$ and the discrete product rules, (2.8) and (2.9), one obtains:

Lemma 2.1. Let $\boldsymbol{u}, \boldsymbol{w} \in \mathcal{H}_{h}$. Then for any vector $\beta=\left\langle\beta_{1}, \beta_{2}\right\rangle$ with $\beta_{1}, \beta_{2} \in \mathbb{R}$

$$
\sum_{i=1}^{N_{1}} \sum_{j=1}^{N_{2}} w_{i, j}\left(\boldsymbol{\beta} \cdot \nabla_{h}^{+}\right) u_{i, j}=-\sum_{i=1}^{N_{1}} \sum_{j=1}^{N_{2}} u_{i, j}\left(\boldsymbol{\beta} \cdot \nabla_{h}^{-}\right) w_{i, j} .
$$


Proof. To prove this, we use the definition of $\mathcal{H}_{h}$.

$$
\begin{aligned}
\sum_{i=1}^{N_{1}} \sum_{j=1}^{N_{2}} w_{i, j}\left(\boldsymbol{\beta} \cdot \nabla_{h}^{+}\right) u_{i, j}= & \sum_{i=1}^{N_{1}} \sum_{j=1}^{N_{2}} w_{i, j}\left(\beta_{1} \nabla_{1, h}^{+} u_{i, j}+\beta_{2} \nabla_{2, h}^{+} u_{i, j}\right) \\
= & \frac{\beta_{1}}{\Delta x}\left(\sum_{i=1}^{N_{1}} \sum_{j=1}^{N_{2}} w_{i, j} u_{i+1, j}-\sum_{i=1}^{N_{1}} \sum_{j=1}^{N_{2}} w_{i, j} u_{i, j}\right)+\frac{\beta_{2}}{\Delta y}\left(\sum_{i=1}^{N_{1}} \sum_{j=1}^{N_{2}} w_{i, j} u_{i, j+1}-\sum_{i=1}^{N_{1}} \sum_{j=1}^{N_{2}} w_{i, j} u_{i, j}\right) \\
= & \frac{\beta_{1}}{\Delta x}\left(\sum_{i=2}^{N_{1}} \sum_{j=1}^{N_{2}} w_{i-1, j} u_{i, j}-\sum_{i=1}^{N_{1}} \sum_{j=1}^{N_{2}} w_{i, j} u_{i, j}\right)+\frac{\beta_{2}}{\Delta y}\left(\sum_{i=1}^{N_{1}} \sum_{j=2}^{N_{2}} w_{i, j-1} u_{i, j}-\sum_{i=1}^{N_{1}} \sum_{j=1}^{N_{2}} w_{i, j} u_{i, j}\right) \\
& +\frac{\beta_{1}}{\Delta x} \sum_{j=1}^{N_{2}} w_{N_{1}, j} u_{1, j}+\frac{\beta_{2}}{\Delta y} \sum_{i=1}^{N_{1}} w_{i, N_{2}} u_{i, 1} \\
= & -\sum_{i=1}^{N_{1}} \sum_{j=1}^{N_{2}} u_{i, j}\left[\beta_{1}\left(\frac{w_{i, j}-w_{i-1, j}}{\Delta x}\right)+\beta_{2}\left(\frac{w_{i, j}-u_{i, j-1}}{\Delta y}\right)\right] \\
= & -\sum_{i=1}^{N_{1}} \sum_{j=1}^{N_{2}} u_{i, j}\left(\beta \cdot \nabla_{h}^{-}\right) w_{i, j} .
\end{aligned}
$$

We define the following discrete semi-norms and norms for $\mathbf{u}=\left(u_{i, j}\right), 1 \leq i \leq N_{1}, 1 \leq j \leq N_{2}$.

$$
\begin{aligned}
|\mathbf{u}|_{1, h} & =\left(\Delta x \Delta y \sum_{i=1}^{N_{1}} \sum_{j=1}^{N_{2}}\left[\left(\nabla_{1, h}^{-} u_{i, j}\right)^{2}+\left(\nabla_{2, h}^{-} u_{i, j}\right)^{2}\right]\right)^{\frac{1}{2}}, \\
|\mathbf{u}|_{2, h} & =\left(\Delta x \Delta y \sum_{i=1}^{N_{1}} \sum_{j=1}^{N_{2}}\left(\Delta_{h} u_{i, j}\right)^{2}\right)^{\frac{1}{2}}, \\
\|\mathbf{u}\|_{\infty, h} & =\max _{\substack{1 \leq i \leq N_{1} \\
1 \leq j \leq N_{2}}}\left|u_{i, j}\right|, \quad\|\mathbf{u}\|_{1, h}^{2}=|\mathbf{u}|_{1, h}^{2}+\|\mathbf{u}\|_{h}^{2} .
\end{aligned}
$$

In 2.10, $\nabla_{1, h}^{-}$and $\nabla_{2, h}^{-}$can be replaced by $\nabla_{1, h}^{+}$and $\nabla_{2, h}^{+}$, respectively. Using 2.10) and 2.11), we have

$$
|\mathbf{u}|_{1, h}^{2} \leq 4\left(\frac{1}{\Delta x^{2}}+\frac{1}{\Delta y^{2}}\right)\|\mathbf{u}\|_{h}^{2}
$$

and the following are obtained by direct computations

$$
\|\mathbf{u}\|_{h}^{2} \leq 4 L_{1} L_{2}\|\mathbf{u}\|_{\infty, h}^{2}
$$

and

$$
\|\mathbf{u}\|_{\infty, h}^{2} \leq \frac{1}{\Delta x \Delta y}\|\mathbf{u}\|_{h}^{2} .
$$

Moreover, it is important to note that if $\mathbf{u}$ belongs to $\mathcal{H}_{h}$, then the discrete Poincaré's inequality holds; this is to say that there is $\eta>0$, independent of $\Delta x$ and $\Delta y$ such that

$$
\eta\|\mathbf{u}\|_{h} \leq|\mathbf{u}|_{1, h}
$$


Remark 2.1. With 2.14, we conclude that the semi-norm $|\cdot|_{1, h}$ is a norm on $\mathcal{H}_{h}$ equivalent to $\|\cdot\|_{1, h}$. The following identities and inequalities will be helpful.

- For any $\mathbf{u}, \mathbf{v} \in \mathcal{H}_{h}$

$$
\begin{aligned}
& 2(\mathbf{u}-\mathbf{v}, \mathbf{u})_{h}=\|\mathbf{u}\|_{h}^{2}-\|\mathbf{v}\|_{h}^{2}+\|\mathbf{u}-\mathbf{v}\|_{h}^{2} \\
& 2(\mathbf{u}-\mathbf{v}, \mathbf{v})_{h}=\|\mathbf{u}\|_{h}^{2}-\|\mathbf{v}\|_{h}^{2}-\|\mathbf{u}-\mathbf{v}\|_{h}^{2}
\end{aligned}
$$

- For $x \in\left[0, \frac{1}{2}\right]$,

$$
\left(\frac{1}{2}\right)^{2 x} \leq 1-x
$$

- Young's inequality: For any $a, b \in \mathbb{R}$ and $\delta>0$, we have

$$
a b \leq \frac{\delta}{2} a^{2}+\frac{1}{2 \delta} b^{2} .
$$

- Cauchy-Schwarz's inequality: For $N \in \mathbb{N}$

$$
\sum_{i=1}^{N} a_{i} b_{i} \leq\left(\sum_{i=1}^{N} a_{i}^{2}\right)^{1 / 2}\left(\sum_{i=1}^{N} b_{i}^{2}\right)^{1 / 2} .
$$

In order to approximate the nonlinear term, we introduce the bilinear map: $C_{h}: \mathcal{H}_{h} \times \mathcal{H}_{h} \rightarrow \mathbb{R}^{N_{1} \times N_{2}}$ in the form

$$
\begin{aligned}
C_{h}(\mathbf{u}, \mathbf{v})_{i, j}= & \alpha_{1}\left[u_{i, j}\left(\boldsymbol{\beta} \cdot \nabla_{h}^{+}\right) v_{i, j}+v_{i, j}\left(\boldsymbol{\beta} \cdot \nabla_{h}^{-}\right) u_{i, j}+v_{i+1, j} \nabla_{1, h}^{+} u_{i, j}+v_{i, j+1} \nabla_{2, h}^{+} u_{i, j}\right] \\
& +\alpha_{2}\left[u_{i, j}\left(\boldsymbol{\beta} \cdot \nabla_{h}^{-}\right) v_{i, j}+v_{i, j}\left(\boldsymbol{\beta} \cdot \nabla_{h}^{+}\right) u_{i, j}+v_{i-1, j} \nabla_{1, h}^{-} u_{i, j}+v_{i, j-1} \nabla_{2, h}^{-} u_{i, j}\right],
\end{aligned}
$$

where $\alpha_{1}$ and $\alpha_{2}$ are constants. We use this bilinear map to approximate the nonlinear term $u(\beta \cdot \nabla) u$ at $t_{n+1}$ and $t_{n}$ for the implicit and explicit methods, respectively.

Using 2.8, 2.9 and Lemma 2.1, we prove the following.

Lemma 2.2. For $\boldsymbol{u}, \boldsymbol{v} \in \mathcal{H}_{h}$

$$
\sum_{i=1}^{N_{1}} \sum_{j=1}^{N_{2}}\left(C_{h}(\boldsymbol{u}, \boldsymbol{v})\right)_{i, j} u_{i, j}=0
$$

Proof. For all $\mathbf{u}, \mathbf{v} \in \mathcal{H}_{h}$, we have

$$
\begin{aligned}
\sum_{i=1}^{N_{1}} \sum_{j=1}^{N_{2}} u_{i, j}\left(\left(\boldsymbol{\beta} \cdot \nabla_{h}^{+}\right) v_{i, j}\right) u_{i, j}= & \sum_{i=1}^{N_{1}} \sum_{j=1}^{N_{2}} u_{i, j}\left(\boldsymbol{\beta} \cdot \nabla_{h}^{+}\right)\left(v_{i, j} u_{i, j}\right)-\sum_{i=1}^{N_{1}} \sum_{j=1}^{N_{2}} u_{i, j}\left(\nabla_{1, h}^{+} u_{i, j}\right) v_{i+1, j} \\
& -\sum_{i=1}^{N_{1}} \sum_{j=1}^{N_{2}} u_{i, j}\left(\nabla_{2, h}^{+} u_{i, j}\right) v_{i, j+1} \quad \text { using 2.8 } \\
= & -\sum_{i=1}^{N_{1}} \sum_{j=1}^{N_{2}} u_{i, j} v_{i, j}\left(\boldsymbol{\beta} \cdot \nabla_{h}^{-}\right)\left(u_{i, j}\right)-\sum_{i=1}^{N_{1}} \sum_{j=1}^{N_{2}} u_{i, j}\left(\nabla_{1, h}^{+} u_{i, j}\right) v_{i+1, j} \\
& -\sum_{i=1}^{N_{1}} \sum_{j=1}^{N_{2}} u_{i, j}\left(\nabla_{2, h}^{+} u_{i, j}\right) v_{i, j+1} \quad \text { using Lemma 2.1 }
\end{aligned}
$$


Similarly

$$
\begin{aligned}
\sum_{i=1}^{N_{1}} \sum_{j=1}^{N_{2}} u_{i, j}\left(\left(\boldsymbol{\beta} \cdot \nabla_{h}^{-}\right) v_{i, j}\right) u_{i, j}= & -\sum_{i=1}^{N_{1}} \sum_{j=1}^{N_{2}} u_{i, j} v_{i, j}\left(\boldsymbol{\beta} \cdot \nabla_{h}^{+}\right)\left(u_{i, j}\right)-\sum_{i=1}^{N_{1}} \sum_{j=1}^{N_{2}} u_{i, j}\left(\nabla_{1, h}^{-} u_{i, j}\right) v_{i-1, j} \\
& -\sum_{i=1}^{N_{1}} \sum_{j=1}^{N_{2}} u_{i, j}\left(\nabla_{2, h}^{-} u_{i, j}\right) v_{i, j-1} .
\end{aligned}
$$

Thus we have

$$
\sum_{i=1}^{N_{1}} \sum_{j=1}^{N_{2}} u_{i, j}\left[u_{i, j}\left(\boldsymbol{\beta} \cdot \nabla_{h}^{+}\right) v_{i, j}+v_{i, j}\left(\boldsymbol{\beta} \cdot \nabla_{h}^{-}\right) u_{i, j}+v_{i+1, j} \nabla_{1, h}^{+} u_{i, j}+v_{i, j+1} \nabla_{2, h}^{+} u_{i, j}\right]=0
$$

and

$$
\sum_{i=1}^{N_{1}} \sum_{j=1}^{N_{2}} u_{i, j}\left[u_{i, j}\left(\boldsymbol{\beta} \cdot \nabla_{h}^{-}\right) v_{i, j}+v_{i, j}\left(\boldsymbol{\beta} \cdot \nabla_{h}^{+}\right) u_{i, j}+v_{i-1, j} \nabla_{1, h}^{-} u_{i, j}+v_{i, j-1} \nabla_{2, h}^{-} u_{i, j}\right]=0
$$

Therefore, the proof is complete.

Remark 2.2. For any d-dimensional space problem with $d \geq 3$, we can easily extend 2.20 such that an analogous of 2.21) holds. That is

$$
\begin{aligned}
C_{h}(\boldsymbol{u}, \boldsymbol{v})_{i_{1}, i_{2}, \cdots, i_{d}}= & \alpha_{1}\left[u_{i_{1}, i_{2}, \cdots, i_{d}}\left(\boldsymbol{\beta} \cdot \nabla_{h}^{+}\right) v_{i_{1}, i_{2}, \cdots, i_{d}}+v_{i_{1}, i_{2}, \cdots, i_{d}}\left(\boldsymbol{\beta} \cdot \nabla_{h}^{-}\right) u_{i_{1}, i_{2}, \cdots, i_{d}}+\sum_{s=1}^{d} v_{s+} \nabla_{s, h}^{+} u_{i_{1}, i_{2}, \cdots, i_{d}}\right] \\
& +\alpha_{2}\left[u_{i_{1}, i_{2}, \cdots, i_{d}}\left(\boldsymbol{\beta} \cdot \nabla_{h}^{-}\right) v_{i_{1}, i_{2}, \cdots, i_{d}}+v_{i_{1}, i_{2}, \cdots, i_{d}}\left(\boldsymbol{\beta} \cdot \nabla_{h}^{+}\right) u_{i_{1}, i_{2}, \cdots, i_{d}}+\sum_{s=1}^{d} v_{s-} \nabla_{s, h}^{-} u_{i_{1}, i_{2}, \cdots, i_{d}}\right]
\end{aligned}
$$

where $v_{s \pm}=v_{i_{1}, \cdots, i_{s} \pm 1, \cdots, i_{d}}$, for $s=1,2, \cdots, d$ and $i_{s}$ is the position of the vector at the $s^{\text {th }}$ coordinate.

Lemma 2.3. For $\boldsymbol{u}, \boldsymbol{w} \in \mathcal{H}_{h}$

$$
\sum_{i=1}^{N_{1}} \sum_{j=1}^{N_{2}} \Delta_{1, h}\left(\Delta_{1, h} u_{i, j}\right) w_{i, j}=\sum_{i=1}^{N_{1}} \sum_{j=1}^{N_{2}} \Delta_{1, h}\left(u_{i, j}\right) \Delta_{1, h}\left(w_{i, j}\right) .
$$

Proof. For all $\mathbf{u}, \mathbf{w} \in \mathcal{H}_{h}$, we have

$$
\begin{aligned}
\sum_{i=1}^{N_{1}} \sum_{j=1}^{N_{2}} \Delta_{1, h}\left(\Delta_{1, h} u_{i, j}\right) w_{i, j}= & \sum_{j=1}^{N_{2}}\left[\sum_{i=1}^{N_{1}} \frac{1}{\Delta x^{4}}\left(u_{i+2, j}-2 u_{i+1, j}+u_{i, j}\right) w_{i, j}-2 \sum_{i=1}^{N_{1}} \frac{1}{\Delta x^{4}}\left(u_{i+1, j}-2 u_{i, j}+u_{i-1, j}\right) w_{i, j}\right. \\
& \left.+\sum_{i=1}^{N_{1}} \frac{1}{\Delta x^{4}}\left(u_{i, j}-2 u_{i-1, j}+u_{i-2, j}\right) w_{i, j}\right]
\end{aligned}
$$

From the periodic boundary conditions, $\sum_{i=1}^{N_{1}} u_{i, j}=\sum_{i=1}^{N_{1}} u_{i-1, j}=\sum_{i=1}^{N_{1}} u_{i+1, j}$ for each $j=1, \ldots, N_{2}$ and hence 2.22 yields

$$
\begin{aligned}
\sum_{i=1}^{N_{1}} \sum_{j=1}^{N_{2}} \Delta_{1, h}\left(\Delta_{1, h} u_{i, j}\right) w_{i, j} & =\sum_{j=1}^{N_{2}}\left[\sum_{i=1}^{N_{1}} \frac{1}{\Delta x^{2}}\left(\Delta_{1, h} u_{i, j}\right) w_{i-1, j}-2 \sum_{i=1}^{N_{1}} \frac{1}{\Delta x^{2}}\left(\Delta_{1, h} u_{i, j}\right) w_{i, j}+\sum_{i=1}^{N_{1}} \frac{1}{\Delta x^{2}}\left(\Delta_{1, h} u_{i, j}\right) w_{i+1, j}\right] \\
& =\sum_{j=1}^{N_{2}} \sum_{i=1}^{N_{1}}\left(\Delta_{1, h} u_{i, j}\right)\left(\frac{1}{\Delta x^{2}}\left(w_{i-1, j}-2 w_{i, j}+w_{i+1, j}\right)\right) \\
& =\sum_{i=1}^{N_{1}} \sum_{j=1}^{N_{2}}\left(\Delta_{1, h} u_{i, j}\right)\left(\Delta_{1, h} w_{i, j}\right) .
\end{aligned}
$$


Lemma 2.3 also holds when one (or two) of the operator(s) $\Delta_{1, h}$ is (are) replaced by $\Delta_{2, h}$.

Lemma 2.4. For $\boldsymbol{u}, \boldsymbol{w} \in \mathcal{H}_{h}$

$$
\sum_{i=1}^{N_{1}} \sum_{j=1}^{N_{2}} \Delta_{h}^{2}\left(u_{i, j}\right) w_{i, j}=\sum_{i=1}^{N_{1}} \sum_{j=1}^{N_{2}} \Delta_{h}\left(u_{i, j}\right) \Delta_{h}\left(w_{i, j}\right) .
$$

Proof. For any $\mathbf{u}, \mathbf{w} \in \mathcal{H}_{h}$ using (2.7), we have

$$
\begin{aligned}
\sum_{i=1}^{N_{1}} \sum_{j=1}^{N_{2}} \Delta_{h}^{2}\left(u_{i, j}\right) w_{i, j}= & \sum_{i=1}^{N_{1}} \sum_{j=1}^{N_{2}}\left[\left(\Delta_{1, h}^{2}+\Delta_{1, h} \Delta_{2, h}+\Delta_{2, h} \Delta_{1, h}+\Delta_{2, h}^{2}\right)\left(u_{i, j}\right)\right] w_{i, j} \\
= & \sum_{i=1}^{N_{1}} \sum_{j=1}^{N_{2}} \Delta_{1, h} u_{i, j} \Delta_{1, h} w_{i, j}+\sum_{i=1}^{N_{1}} \sum_{j=1}^{N_{2}} \Delta_{2, h} u_{i, j} \Delta_{1, h} w_{i, j}+\sum_{i=1}^{N_{1}} \sum_{j=1}^{N_{2}} \Delta_{1, h} u_{i, j} \Delta_{2, h} w_{i, j} \\
& \quad+\sum_{i=1}^{N_{1}} \sum_{j=1}^{N_{2}} \Delta_{2, h} u_{i, j} \Delta_{2, h} w_{i, j} \quad \text { using Lemma } 2.3 \\
= & \sum_{i=1}^{N_{1}} \sum_{j=1}^{N_{2}}\left(\Delta_{1, h} u_{i, j}+\Delta_{2, h} u_{i, j}\right)\left(\Delta_{1, h} w_{i, j}+\Delta_{2, h} w_{i, j}\right) \\
= & \sum_{i=1}^{N_{1}} \sum_{j=1}^{N_{2}} \Delta_{h} u_{i, j} \Delta_{h} w_{i, j} .
\end{aligned}
$$

The following lemma will be used later.

Lemma 2.5. For $\boldsymbol{u} \in\left\{\boldsymbol{u}=(u)_{i, j}, u_{i, j} \in \mathbb{R} \mid u_{i+N_{1}, j}=u_{i, j}=u_{i, j+N_{2}}, i, j \in \mathbb{Z}\right\}$, the following inequality holds true

$$
|\boldsymbol{u}|_{1, h}^{2} \leq|\boldsymbol{u}|_{2, h}\|\boldsymbol{u}\|_{h}
$$

Proof. Using 2.7], for $\mathbf{u} \in\left\{\mathbf{u}=(u)_{i, j}, u_{i, j} \in \mathbb{R} \mid u_{i+N_{1}, j}=u_{i, j}=u_{i, j+N_{2}}, i, j \in \mathbb{Z}\right\}$, we have

$$
\begin{aligned}
\left(\Delta_{h} \mathbf{u}, \mathbf{u}\right)_{h}= & \left(\Delta_{1, h} \mathbf{u}+\Delta_{2, h} \mathbf{u}, \mathbf{u}\right)_{h} \\
= & \sum_{i=1}^{N_{1}} \sum_{j=1}^{N_{2}}\left[\frac{u_{i+1, j}-2 u_{i, j}+u_{i-1, j}}{\Delta x^{2}}+\frac{u_{i, j+1}-2 u_{i, j}+u_{i, j-1}}{\Delta y^{2}}\right] u_{i, j} \\
= & \sum_{i=1}^{N_{1}} \sum_{j=1}^{N_{2}}\left(\frac{u_{i+1, j}-u_{i, j}}{\Delta x^{2}}\right) u_{i, j}-\sum_{i=1}^{N_{1}} \sum_{j=1}^{N_{2}}\left(\frac{u_{i, j}-u_{i-1, j}}{\Delta x^{2}}\right) u_{i, j} \\
& +\sum_{i=1}^{N_{1}} \sum_{j=1}^{N_{2}}\left(\frac{u_{i, j+1}-u_{i, j}}{\Delta y^{2}}\right) u_{i, j}-\sum_{i=1}^{N_{1}} \sum_{j=1}^{N_{2}}\left(\frac{u_{i, j}-u_{i, j-1}}{\Delta y^{2}}\right) u_{i, j} .
\end{aligned}
$$

Using periodicity, we obtain

$$
\left(\Delta_{h} \mathbf{u}, \mathbf{u}\right)_{h}=-\sum_{i=1}^{N_{1}} \sum_{j=1}^{N_{2}}\left(\frac{u_{i, j}-u_{i-1, j}}{\Delta x}\right)^{2}-\sum_{i=1}^{N_{1}} \sum_{j=1}^{N_{2}}\left(\frac{u_{i, j}-u_{i, j-1}}{\Delta y}\right)^{2}=-|\mathbf{u}|_{1, h}^{2} .
$$


Using Cauchy-Schwarz's inequality, we have

$$
|\mathbf{u}|_{1, h}^{2}=\leq\left\|\Delta_{h} \mathbf{u}\right\|_{h}\|\mathbf{u}\|_{h}=|\mathbf{u}|_{2, h}\|\mathbf{u}\|_{h} .
$$

For $\mathbf{u} \in \mathcal{H}_{h}$, Lemma 2.5 and Young's inequality implies the existence of $\eta$, positive constant independent of both $\Delta y$, and $\Delta x$ such that

$$
\eta|\mathbf{u}|_{1, h} \leq|\mathbf{u}|_{2, h}
$$

\section{One-level Finite Volume Methods}

In this section, we present two traditional one-level finite volume methods: namely implicit finite volume method and explicit finite volume method. The existence, uniqueness and convergence of solution for the implicit method are proved and stability analysis is examined for both schemes. For both methods thirteen point stencils are used to approximate (1.1)-(1.4), as shown in Fig. 1. The introduction of these classical schemes is important at least for three reasons:

(a) comparison with multilevel methods;

(b) these schemes that are categorized as classical present significant challenges for their analysis as we will see;

(c) the analysis of these schemes will shed lights in the analysis of multilevel methods.

\subsection{Implicit one-level finite volume method}

The nonlinear term $u(\boldsymbol{\beta} \cdot \nabla u)$ at $t_{n+1}$ is approximated linearly using the bilinear map defined in section 2 . 2.20 , and is given by

$$
\left.[u(\boldsymbol{\beta} \cdot \nabla) u]\right|_{i, j} ^{n+1} \approx\left(C_{h}\left(\mathbf{u}^{n+1}, \tilde{\mathbf{u}}^{n}\right)\right)_{i, j},
$$

where $\tilde{\mathbf{u}}^{n}$ is the approximation of $\mathbf{u}^{n+1}$, given by

$$
\tilde{\mathbf{u}}^{n}=a_{1} \mathbf{u}^{n}+a_{2} \mathbf{u}^{n-1}+a_{3} \mathbf{u}^{n-2}+\cdots+a_{m_{0}} \mathbf{u}^{n-m_{0}+1},
$$

where $m_{0} \in\{1,2, \ldots, n\}$ and $a_{1}, a_{2}, \ldots$, and $a_{m_{0}}$ are coefficients that determine the approximation with $3\left(\alpha_{1}+\alpha_{2}\right)\left(a_{1}+a_{2}+\cdots+a_{m_{0}}\right)=1$, ensuring consistency of the approximation. For $m<m_{0}-1$, the term $\tilde{\mathbf{u}}^{m}$ is given by the relation

$$
\tilde{\mathbf{u}}^{m}=\mathbf{u}^{m}
$$

We approximate the nonlinear term on the right hand side of 1.1 at $t_{n+1}$ by a linear second order accurate in space as follows:

$$
\left.\Delta f(u)\right|_{i, j} ^{n+1} \approx \nabla_{1, h}^{+}\left(\varphi_{i-1 / 2, j}^{n} \nabla_{1, h}^{-} u_{i, j}^{n+1}\right)+\nabla_{2, h}^{+}\left(\varphi_{i, j-1 / 2}^{n} \nabla_{2, h}^{-} u_{i, j}^{n+1}\right) .
$$

where

$$
\varphi_{i-1 / 2, j}^{n}=\frac{f^{\prime}\left(u_{i, j}^{n}\right)+f^{\prime}\left(u_{i-1, j}^{n}\right)}{2} \text { and } \varphi_{i, j-1 / 2}^{n}=\frac{f^{\prime}\left(u_{i, j}^{n}\right)+f^{\prime}\left(u_{i, j-1}^{n}\right)}{2} .
$$

Lemma 3.1. For $\boldsymbol{u}^{n}, \boldsymbol{u}^{n+1} \in \mathcal{H}_{h}$

$$
\Delta x \Delta y \sum_{i=1}^{N_{1}} \sum_{j=1}^{N_{2}}\left[\nabla_{1, h}^{+}\left(\varphi_{i-1 / 2, j}^{n} \nabla_{1, h}^{-} u_{i, j}^{n+1}\right)+\nabla_{2, h}^{+}\left(\varphi_{i, j-1 / 2}^{n} \nabla_{2, h}^{-} u_{i, j}^{n+1}\right)\right] u_{i, j}^{n+1} \leq\left|\boldsymbol{u}^{n+1}\right|_{1, h}^{2} .
$$




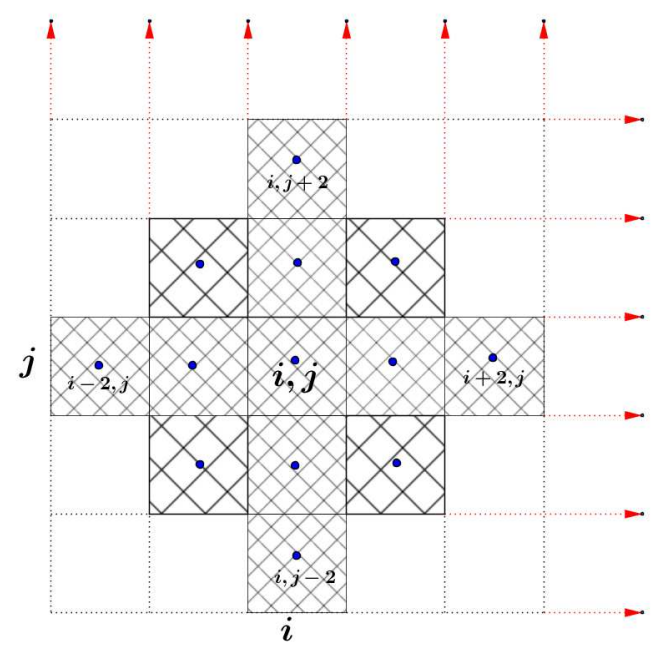

Figure 1: Finite volume discretization in 2D

Proof. For $\mathbf{u}^{n}, \mathbf{u}^{n+1} \in \mathcal{H}_{h}$, applying Lemma 2.1, we have

$$
\begin{gathered}
\Delta x \Delta y \sum_{i=1}^{N_{1}} \sum_{j=1}^{N_{2}} \nabla_{1, h}^{+}\left(\varphi_{i-1 / 2, j}^{n} \nabla_{1, h}^{-} u_{i, j}^{n+1}\right) u_{i, j}^{n+1}+\nabla_{2, h}^{+}\left(\varphi_{i, j-1 / 2}^{n} \nabla_{2, h}^{-} u_{i, j}^{n+1}\right) u_{i, j}^{n+1} \\
=-\Delta x \Delta y \sum_{i=1}^{N_{1}} \sum_{j=1}^{N_{2}}\left[\varphi_{i-1 / 2, j}^{n}\left(\nabla_{1, h}^{-} u_{i, j}^{n+1}\right)^{2}+\varphi_{i, j-1 / 2}^{n}\left(\nabla_{2, h}^{-} u_{i, j}^{n+1}\right)^{2}\right] \\
=-\frac{3}{2} \Delta x \Delta y \sum_{i=1}^{N_{1}} \sum_{j=1}^{N_{2}}\left[\left(\left(u_{i, j}^{n}\right)^{2}+\left(u_{i-1, j}^{n}\right)^{2}\right)\left(\nabla_{1, h}^{-} u_{i, j}^{n+1}\right)^{2}+\left(\left(u_{i, j}^{n}\right)^{2}+\left(u_{i, j-1}^{n}\right)^{2}\right)\left(\nabla_{2, h}^{-} u_{i, j}^{n+1}\right)^{2}\right] \\
\quad+\Delta x \Delta y \sum_{i=1}^{N_{1}} \sum_{j=1}^{N_{2}}\left[\left(\nabla_{1, h}^{-} u_{i, j}^{n+1}\right)^{2}+\left(\nabla_{2, h}^{-} u_{i, j}^{n+1}\right)^{2}\right] \\
\leq \Delta x \Delta y \sum_{i=1}^{N_{1}} \sum_{j=1}^{N_{2}}\left[\left(\nabla_{1, h}^{-} u_{i, j}^{n+1}\right)^{2}+\left(\nabla_{2, h}^{-} u_{i, j}^{n+1}\right)^{2}\right]=\left|\mathbf{u}^{n+1}\right|_{1, h}^{2} .
\end{gathered}
$$

The fourth order derivative is discretized using the central difference method and combining together with (3.1) and (3.4), the implicit one-level finite volume discretization of (1.1)-(1.4) is given as follows:

$$
\begin{aligned}
& \frac{u_{i, j}^{n+1}-u_{i, j}^{n}}{\Delta t}-C_{h}\left(\mathbf{u}^{n+1}, \tilde{\mathbf{u}}^{n}\right)_{i, j}+\varepsilon^{2} \Delta_{h}^{2} u_{i, j}^{n+1}=\nabla_{1, h}^{+}\left(\varphi_{i-1 / 2, j}^{n} \nabla_{1, h}^{-} u_{i, j}^{n+1}\right)+\nabla_{2, h}^{+}\left(\varphi_{i, j-1 / 2}^{n} \nabla_{2, h}^{-} u_{i, j}^{n+1}\right), \\
& u_{i, j}^{n}=u_{i+N_{1}, j}^{n}=u_{i, j+N_{2}}^{n} \\
& u_{i, j}^{0}=\frac{1}{\Delta x \Delta y} \iint_{k_{i, j}} u^{0}(x) d x d y .
\end{aligned}
$$

Remark 3.1. It is worth noting thanks to 2.20 , that $3.5 \mathrm{a})-(3.5 \mathrm{c})$ is a linear system of equations, while (1.1)-(1.4) is nonlinear. 
Before discussing some qualitative properties of the solution of $3.5 \mathrm{a})-3.5 \mathrm{c}$, we first address its feasibility.

Theorem 3.1. If $\Delta t<4 \varepsilon^{2}$, then the approximate solution $\boldsymbol{u}^{n}$ of (3.5a)-(3.5c) is unique.

Noting that Equations (3.5a)-(3.5c) is a linear system in finite dimensional space, its existence is equivalent to uniqueness of solution 30 . Thus, we only show that the approximations $\mathbf{u}^{1}, \mathbf{u}^{2}, \ldots, \mathbf{u}^{M}$ satisfying (3.5a)(3.5c), are unique.

Proof. For $n=0,1,2, \ldots, M$, let $\mathbf{v}^{n}$ and $\mathbf{u}^{n}$ be two sequences of solutions of $(3.5 \mathrm{a})-(3.5 \mathrm{c})$ with $\mathbf{v}_{0}=\mathbf{u}_{0}$. Let $\mathbf{z}^{n}=\mathbf{u}^{n}-\mathbf{v}^{n}$ and clearly $\mathbf{z}^{0}=0$. We shall prove by induction that $\mathbf{z}^{n}=0$ for all $n=1,2, \ldots, M$. We observe that $\mathbf{z}^{n+1}$ is a solution of

$$
\begin{aligned}
\frac{1}{\Delta t}\left(z_{i, j}^{n+1}-z_{i, j}^{n}\right)-\left(C_{h}\left(\mathbf{u}^{n+1}, \tilde{\mathbf{u}}^{n}\right)\right)_{i, j}+ & \left(C_{h}\left(\mathbf{v}^{n+1}, \tilde{\mathbf{v}}^{n}\right)\right)_{i, j}+\varepsilon^{2} \Delta_{h}^{2} z_{i, j}^{n+1} \\
= & \nabla_{1, h}^{+}\left(\varphi_{i-1 / 2, j}^{n} \nabla_{1, h}^{-} u_{i, j}^{n+1}\right)+\nabla_{2, h}^{+}\left(\varphi_{i, j-1 / 2}^{n} \nabla_{2, h}^{-} u_{i, j}^{n+1}\right) \\
& -\nabla_{1, h}^{+}\left(\psi_{i-1 / 2, j}^{n} \nabla_{1, h}^{-} v_{i, j}^{n+1}\right)+\nabla_{2, h}^{+}\left(\psi_{i, j-1 / 2}^{n} \nabla_{2, h}^{-} v_{i, j}^{n+1}\right),
\end{aligned}
$$

for $i=1, \cdots, N_{1}, j=1, \cdots, N_{2}$ and

$$
\psi_{i-1 / 2, j}^{n}=\frac{f^{\prime}\left(v_{i, j}^{n}\right)+f^{\prime}\left(v_{i-1, j}^{n}\right)}{2} \text { and } \psi_{i, j-1 / 2}^{n}=\frac{f^{\prime}\left(v_{i, j}^{n}\right)+f^{\prime}\left(v_{i, j-1}^{n}\right)}{2} .
$$

By induction, we assume that $\mathbf{z}^{n}=0$ and we want to show that $\mathbf{z}^{n+1}=0$. It follows then that

$$
z_{i, j}^{n+1}-\Delta t\left(C_{h}\left(\mathbf{z}^{n+1}, \tilde{\mathbf{u}}^{n}\right)\right)_{i, j}+\Delta t \varepsilon^{2} \Delta_{h}^{2} z_{i, j}^{n+1}=\Delta t\left[\nabla_{1, h}^{+}\left(\varphi_{i-1 / 2, j}^{n} \nabla_{1, h}^{-} z_{i, j}^{n+1}\right)+\nabla_{2, h}^{+}\left(\varphi_{i, j-1 / 2}^{n} \nabla_{2, h}^{-} z_{i, j}^{n+1}\right)\right] .
$$

Multiplying 3.7 by $\Delta t \Delta x \Delta y z_{i . j}^{n+1}$ and summing the resulting equalities for $i=1, \ldots, N_{1}, j=1, \ldots, N_{2}$, with help of 2.21 and Lemma 2.1 we obtain

$$
\left\|\mathbf{z}^{n+1}\right\|_{h}^{2}+\Delta t \varepsilon^{2}\left|\mathbf{z}^{n+1}\right|_{2, h}^{2}=-\Delta t \Delta x \Delta y \sum_{i=1}^{N_{1}} \sum_{j=1}^{N_{2}}\left[\varphi_{i-1 / 2, j}^{n}\left(\nabla_{1, h}^{-} z_{i, j}^{n+1}\right)^{2}+\varphi_{i, j-1 / 2}^{n}\left(\nabla_{2, h}^{-} z_{i, j}^{n+1}\right)^{2}\right],
$$

which after the application of Lemma 3.1 gives

$$
\left\|\mathbf{z}^{n+1}\right\|_{h}^{2}+\Delta t \varepsilon^{2}\left|\mathbf{z}^{n+1}\right|_{2, h}^{2} \leq \Delta t\left|\mathbf{z}^{n+1}\right|_{1, h}^{2} .
$$

Applying Lemma 2.5 and Young's inequality, 3.8 implies that

$$
\left(1-\frac{\Delta t}{4 \varepsilon^{2}}\right)\left\|\mathbf{z}^{n+1}\right\|_{h}^{2} \leq 0 .
$$

For $\frac{\Delta t}{4 \varepsilon^{2}}<1$, we get

$$
\left\|\mathbf{z}^{n+1}\right\|_{h}^{2} \leq 0
$$

Therefore, $\mathbf{z}^{n+1}=0$. This completes the proof of uniqueness, hence the existence of solution.

With the existence of solution being conditioned, it is quite clear that all possible results will be under at least the same condition, that is $\Delta t<4 \varepsilon^{2}$. About the stability of the method (3.5a)- $\left.3.5 \mathrm{c}\right)$, we have the following theorem.

Theorem 3.2. The finite volume method defined by 3.5a)-(3.5c), is conditionally stable in $L^{\infty}\left(0, T ; \mathcal{H}_{h}\right)$, that is, for $\Delta t \leq \varepsilon^{2}$ and $1 \leq n \leq M$,

$$
\left\|\boldsymbol{u}^{n}\right\|_{h}^{2} \leq 2 \frac{2 T}{\varepsilon^{2}}\left\|\boldsymbol{u}^{0}\right\|_{h}^{2}
$$


Proof. By multiplying 3.5a with $2 \Delta x \Delta y \Delta t u_{i, j}^{n+1}$ and summing from $i=1$ to $N_{1}$ and $j=1$ to $N_{2}$, we obtain

$$
\begin{aligned}
\left\|\mathbf{u}^{n+1}\right\|_{h}^{2} & -\left\|\mathbf{u}^{n}\right\|_{h}^{2}+\left\|\mathbf{u}^{n+1}-\mathbf{u}^{n}\right\|_{h}^{2}+2 \Delta t \varepsilon^{2}\left(\Delta_{h}^{2} \mathbf{u}^{n+1}, \mathbf{u}^{n+1}\right)_{h} \\
& =2 \Delta x \Delta y \Delta t \sum_{i=1}^{N_{1}} \sum_{i=1}^{N_{2}}\left[\nabla_{1, h}^{+}\left(\varphi_{i-1 / 2, j}^{n} \nabla_{1, h}^{-} u_{i, j}^{n+1}\right)+\nabla_{2, h}^{+}\left(\varphi_{i, j-1 / 2}^{n} \nabla_{2, h}^{-} u_{i, j}^{n+1}\right)\right] u_{i, j}^{n+1} .
\end{aligned}
$$

Using Lemmas 2.4 and 3.1 together with 3.10 , we have

$$
\left\|\mathbf{u}^{n+1}\right\|_{h}^{2}-\left\|\mathbf{u}^{n}\right\|_{h}^{2}+\left\|\mathbf{u}^{n+1}-\mathbf{u}^{n}\right\|_{h}^{2}+2 \Delta t \varepsilon^{2}\left|\mathbf{u}^{n+1}\right|_{2, h}^{2} \leq 2 \Delta t\left|\mathbf{u}^{n+1}\right|_{1, h}^{2} .
$$

Due to Lemma 2.5. Young's inequality and dropping the term $\left\|\mathbf{u}^{n+1}-\mathbf{u}^{n}\right\|_{h}^{2}$, (3.11) gives

$$
\left\|\mathbf{u}^{n+1}\right\|_{h}^{2}-\left\|\mathbf{u}^{n}\right\|_{h}^{2} \leq \frac{\Delta t}{2 \varepsilon^{2}}\left\|\mathbf{u}^{n+1}\right\|_{h}^{2}
$$

which is re-written as follows

$$
\left[1-\frac{\Delta t}{2 \varepsilon^{2}}\right]\left\|\mathbf{u}^{n+1}\right\|_{h}^{2} \leq\left\|\mathbf{u}^{n}\right\|_{h}^{2}
$$

Based on 2.17, for $\frac{\Delta t}{2 \varepsilon^{2}} \leq \frac{1}{2}, 3.12$ gives

$$
\left\|\mathbf{u}^{n+1}\right\|_{h}^{2} \leq 2 \frac{2 \Delta t}{\varepsilon^{2}}\left\|\mathbf{u}^{n}\right\|_{h}^{2} .
$$

By induction over $n$, we obtain

$$
\left\|\mathbf{u}^{n}\right\|_{h}^{2} \leq 2 \frac{2 n \Delta t}{\varepsilon^{2}}\left\|\mathbf{u}^{0}\right\|_{h}^{2} \leq 2 \frac{2 T}{\varepsilon^{2}}\left\|\mathbf{u}^{0}\right\|_{h}^{2} .
$$

Therefore, the proof is complete.

Remark 3.2. Starting with (3.11) and Lemma 2.5, one has

$$
\left\|\boldsymbol{u}^{n+1}\right\|_{h}^{2}-\left\|\boldsymbol{u}^{n}\right\|_{h}^{2}+\left\|\boldsymbol{u}^{n+1}-\boldsymbol{u}^{n}\right\|_{h}^{2}+2 \Delta t \varepsilon^{2} \eta^{2}\left|\boldsymbol{u}^{n+1}\right|_{1, h}^{2} \leq 2 \Delta t\left|\boldsymbol{u}^{n+1}\right|_{1, h}^{2} .
$$

Hence, we obtain

$$
\left\|\boldsymbol{u}^{n+1}\right\|_{h}^{2}+2 \Delta t\left(\varepsilon^{2} \eta^{2}-1\right)\left|\boldsymbol{u}^{n+1}\right|_{1, h}^{2} \leq\left\|\boldsymbol{u}^{n}\right\|_{h}^{2},
$$

from which one deduces that if $\varepsilon$ is big enough, then

$$
\left\|\boldsymbol{u}^{n+1}\right\|_{h}^{2} \leq\left\|\boldsymbol{u}^{n}\right\|_{h}^{2} \leq\left\|\boldsymbol{u}^{n-1}\right\|_{h}^{2} \leq \cdots \leq\left\|\boldsymbol{u}^{0}\right\|_{h}^{2}
$$

This alternative stability result requires both that $\Delta t<4 \varepsilon^{2}$ and the viscosity constant $\varepsilon$ big enough.

Theorem 3.3. Suppose that the solution $u(x, t)$ of (1.1)-(1.4) is sufficiently smooth. Assume that $\Delta t<\min \left(4 \varepsilon^{2}, c\right)$, with $c$ given by 3.43$)$, independent of $\Delta x$ and $\Delta y$. Assume that $\Delta t, \Delta x$ and $\Delta y$ satisfy the relation (3.45).

Then, the solution of the finite volume discretization (3.5a)-(3.5c) converges to the solution of the problems (1.1) -(1.4) in the discrete $L^{2}$-norm with rate of convergence $\mathcal{O}\left(\Delta t+\Delta x^{2}+\Delta y^{2}\right)$. 
Proof. For $i=1, \ldots, N_{1}$ and $j=1, \ldots, N_{2}$, let

$$
v_{i, j}^{n}=\frac{1}{\Delta x \Delta y} \iint_{k_{i, j}} u\left(x, y, t_{n}\right) d x d y
$$

be the cell average of the exact solution $u$ of (1.1)-(1.4) at time $t_{n}$, for $0 \leq n \leq M$, on the cell $k_{i, j}$. Since $u$ is smooth enough by assumption, (hence at least continuous on $\left[-L_{1}, L_{1}\right] \times\left[-L_{2}, L_{2}\right]$ ) we let

$$
s=\max _{-L_{1} \leq x \leq L_{1},-L_{2} \leq y \leq L_{2}, 0 \leq t \leq T}|u(x, y, t)| .
$$

Also, the smoothness of $u$ gives

$$
v_{i, j}^{n}=u\left(x_{i}, y_{j}, t_{n}\right)+\mathcal{O}\left(\Delta x^{2}+\Delta y^{2}\right), \quad 1 \leq i \leq N_{1}, 1 \leq j \leq N_{2} \text { and } 0 \leq n \leq M .
$$

Making use of Taylor's expansion (see Appendix A, we obtain

$$
\begin{aligned}
& \frac{v_{i, j}^{n+1}-v_{i, j}^{n}}{\Delta t}=\left.u_{t}\right|_{i, j} ^{n}+\mathcal{O}\left(\Delta t+\Delta x^{2}+\Delta y^{2}\right), \\
& \Delta_{h}^{2} v_{i, j}^{n+1}=\left.\Delta^{2} u\right|_{i, j} ^{n}+\mathcal{O}\left(\Delta t+\Delta x^{2}+\Delta y^{2}\right), \\
& \nabla_{1, h}^{+}\left(\psi_{i-\frac{1}{2}, j}^{n} \nabla_{1, h}^{-} v_{i, j}^{n+1}\right)+\nabla_{2, h}^{+}\left(\psi_{i, j-\frac{1}{2}}^{n} \nabla_{2, h}^{-} v_{i, j}^{n+1}\right)=\left.\Delta f(u)\right|_{i, j} ^{n}+\mathcal{O}\left(\Delta t+\Delta x^{2}+\Delta y^{2}\right),
\end{aligned}
$$

and

$$
\begin{aligned}
\left(C_{h}\left(\boldsymbol{v}^{n+1}, \tilde{\boldsymbol{v}}^{n}\right)\right)_{i, j}= & \left(\alpha_{1}-\alpha_{2}\right)\left(a_{1}+a_{2}+\cdots+a_{m_{0}}\right)\left(\left.\Delta x\left(u_{x}^{2}\right)\right|_{i, j} ^{n}+\left.\Delta y\left(u_{y}^{2}\right)\right|_{i, j} ^{n}\right) \\
& +\frac{1}{2}\left(\alpha_{1}-\alpha_{2}\right)\left(a_{1}+a_{2}+\cdots+a_{m_{0}}\right)\left(\left.\Delta x\left(u u_{x x}\right)\right|_{i, j} ^{n}+\left.\Delta y\left(u u_{y y}\right)\right|_{i, j} ^{n}\right) \\
& +\left.3\left(\alpha_{1}+\alpha_{2}\right)\left(a_{1}+a_{2}+\cdots+a_{m_{0}}\right)(u(\boldsymbol{\beta} \cdot \nabla) u)\right|_{i, j} ^{n}+\mathcal{O}\left(\Delta t+\Delta x^{2}+\Delta y^{2}\right) .
\end{aligned}
$$

One observes that the numerical scheme is first order accurate when $\alpha_{1} \neq \alpha_{2}$ and it is second order accurate in space if $\alpha_{1}=\alpha_{2}$, from which 3.19 gives

$$
C_{h}\left(\boldsymbol{v}^{n+1}, \tilde{\boldsymbol{v}}^{n}\right)_{i, j}=\left.(u(\boldsymbol{\beta} \cdot \nabla) u)\right|_{i, j} ^{n}+\mathcal{O}\left(\Delta t+\Delta x^{2}+\Delta y^{2}\right) .
$$

In this study, we consider the case $\alpha_{1}=\alpha_{2}$ to obtain second order accurate method. Combining (3.16)- 3.18 and $(3.20)$, we obtain

$$
\left\{\begin{array}{l}
\frac{v_{i, j}^{n+1}-v_{i, j}^{n}}{\Delta t}-\left(C_{h}\left(\boldsymbol{v}^{n+1}, \tilde{\boldsymbol{v}}^{n}\right)\right)_{i, j}+\varepsilon^{2} \Delta_{h}^{2} v_{i, j}^{n+1}=\nabla_{1, h}^{+}\left(\psi_{i-\frac{1}{2}, j}^{n} \nabla_{1, h}^{-} v_{i, j}^{n+1}\right)+\nabla_{2, h}^{+}\left(\psi_{i, j-\frac{1}{2}}^{n} \nabla_{2, h}^{-} v_{i, j}^{n+1}\right)+r_{i, j}^{n}, \\
v_{i, j}^{0}=\frac{1}{\Delta x \Delta y} \iint_{k_{i, j}} u^{0}(x, y) d x d y
\end{array}\right.
$$

where $r_{i, j}^{n}$ is the truncation error of the finite volume discretization $3.5 \mathrm{a}$ for $0 \leq n \leq M-1,1 \leq i \leq N_{1}$ and $1 \leq j \leq N_{2}$. There exists a positive constant $c_{1}$ such that

$$
\max _{i, j, n}\left|r_{i, j}^{n}\right| \leq c_{1}\left(\Delta t+\Delta x^{2}+\Delta y^{2}\right), 0 \leq n \leq M-1,1 \leq i \leq N_{1}, 1 \leq j \leq N_{2} .
$$

Let $\mathbf{e}^{n}=\boldsymbol{v}^{n}-\mathbf{u}^{n}, 0 \leq n \leq M$, where $u_{i, j}^{n}$ is the solution of $3.5 \mathrm{a}$ - $3.5 \mathrm{c}$. Clearly $\mathbf{e}^{n} \in \mathcal{H}_{h}$ and $\mathbf{e}^{0}=0$.

Substituting $u_{i, j}^{n}=v_{i, j}^{n}-e_{i, j}^{n}$ into (3.5a), and using (3.21), we obtain

$$
\begin{aligned}
\frac{e_{i, j}^{n+1}-e_{i, j}^{n}}{\Delta t} & -\left(C_{h}\left(\mathbf{e}^{n+1}, \tilde{\boldsymbol{v}}^{n}-\tilde{\mathbf{e}}^{n}\right)\right)_{i, j}+\varepsilon^{2} \Delta_{h}^{2} e_{i, j}^{n+1}=\nabla_{1, h}^{+}\left(\varphi_{i-\frac{1}{2}, j}^{n} \nabla_{1, h}^{-} e_{i, j}^{n+1}\right)+\nabla_{2, h}^{+}\left(\varphi_{i, j-\frac{1}{2}}^{n} \nabla_{2, h}^{-} e_{i, j}^{n+1}\right) \\
& +\nabla_{1, h}^{+}\left[\left(3\left(v_{i, j}^{n} e_{i, j}^{n}+v_{i-1, j}^{n} e_{i-1, j}^{n}\right)-\frac{3}{2}\left[\left(e_{i, j}^{n}\right)^{2}+\left(e_{i-1, j}^{n}\right)^{2}\right]\right) \nabla_{1, h}^{-} v_{i, j}^{n+1}\right]+\left(C_{h}\left(\boldsymbol{v}^{n+1}, \tilde{\mathbf{e}}^{n}\right)\right)_{i, j} \\
& +\nabla_{2, h}^{+}\left[\left(3\left(v_{i, j}^{n} e_{i, j}^{n}+v_{i, j-1}^{n} e_{i, j-1}^{n}\right)-\frac{3}{2}\left[\left(e_{i, j}^{n}\right)^{2}+\left(e_{i, j-1}^{n}\right)^{2}\right]\right) \nabla_{2, h}^{-} v_{i, j}^{n+1}\right]+r_{i, j}^{n}
\end{aligned}
$$


where

$$
\psi_{i-1 / 2, j}^{n}=\frac{f^{\prime}\left(v_{i, j}^{n}\right)+f^{\prime}\left(v_{i-1, j}^{n}\right)}{2} \text { and } \psi_{i, j-1 / 2}^{n}=\frac{f^{\prime}\left(v_{i, j}^{n}\right)+f^{\prime}\left(v_{i, j-1}^{n}\right)}{2} .
$$

Multiplying 3.23 by $2 \Delta t \Delta x \Delta y e_{i, j}^{n+1}$ and summing the corresponding equalities for $i=1, \ldots, N_{1}$ and $j=1, \ldots, N_{2}$, together with 2.15 and Lemmas 2.1 and 3.1. we obtain

$$
\begin{aligned}
& \left\|\mathbf{e}^{n+1}\right\|_{h}^{2}-\left\|\mathbf{e}^{n}\right\|_{h}^{2}+\left\|\mathbf{e}^{n+1}-\mathbf{e}^{n}\right\|_{h}^{2}+2 \Delta t \varepsilon^{2}\left|\mathbf{e}^{n+1}\right|_{2, h}^{2} \leq 2 \Delta t\left|\mathbf{e}^{n+1}\right|_{1, h}^{2} \\
& \quad-6 \Delta t \Delta x \Delta \sum_{i=1}^{N_{1}} \sum_{j=1}^{N_{2}}\left[\left(v_{i, j}^{n} e_{i, j}^{n}+v_{i-1, j}^{n} e_{i-1, j}^{n}\right) \nabla_{1, h}^{-} v_{i, j}^{n+1} \nabla_{1, h}^{-} e_{i, j}^{n+1}+\left(v_{i, j}^{n} e_{i, j}^{n}+v_{i, j-1}^{n} e_{i, j-1}^{n}\right) \nabla_{2, h}^{-} v_{i, j}^{n+1} \nabla_{2, h}^{-} e_{i, j}^{n+1}\right] \\
& \quad+3 \Delta t \Delta x \Delta y \sum_{i=1}^{N_{1}} \sum_{j=1}^{N_{2}}\left[\left[\left(e_{i, j}^{n}\right)^{2}+\left(e_{i-1, j}^{n}\right)^{2}\right] \nabla_{1, h}^{-} v_{i, j}^{n+1} \nabla_{1, h}^{-} e_{i, j}^{n+1}+\left[\left(e_{i, j}^{n}\right)^{2}+\left(e_{i, j-1}^{n}\right)^{2}\right] \nabla_{2, h}^{-} v_{i, j}^{n+1} \nabla_{2, h}^{-} e_{i, j}^{n+1}\right] \\
& \quad+2 \Delta t\left(C_{h}\left(\boldsymbol{v}^{n+1}, \tilde{\mathbf{e}}^{n}\right), \mathbf{e}^{n+1}\right)_{h}+2 \Delta t\left(\mathbf{r}^{n}, \mathbf{e}^{n+1}\right)_{h} \\
& \quad=\sum_{m=1}^{7} I_{m} .
\end{aligned}
$$

We estimate each of the terms $I_{2}, \ldots, I_{7}(3.24)$ as follows:

$$
I_{2} \leq 6 \Delta t\left[\left(\max _{\substack{1 \leq i \leq N_{1} \\ 1 \leq j \leq N_{2}}}\left|v_{i, j}^{n}\right|\right)\left(\max _{\substack{1 \leq i \leq N_{1} \\ 1 \leq j \leq N_{2}}}\left|\nabla_{1, h}^{-} v_{i, j}^{n}\right|\right)\right]\left(\Delta x \Delta y \sum_{i=1}^{N_{1}} \sum_{j=1}^{N_{2}}\left|e_{i, j}^{n}\right|\left|\nabla_{1, h}^{-} e_{i, j}^{n+1}\right|+\left|e_{i-1, j}^{n}\right|\left|\nabla_{1, h}^{-} e_{i, j}^{n+1}\right|\right)
$$

Since $u$ is smooth then there exist constants $c_{2}$ and $c_{3}$ such that

$$
\max _{\substack{1 \leq i \leq N_{1} \\ 1 \leq j \leq N_{2}}}\left|\nabla_{1, h}^{-} v_{i, j}^{n+1}\right| \leq c_{2} \text { and } \max _{\substack{1 \leq i \leq N_{1} \\ 1 \leq j \leq N_{2}}}\left|\nabla_{2, h}^{-} v_{i, j}^{n+1}\right| \leq c_{3} \quad \forall n=0,1, \cdots, M-1 .
$$

Thus,

$$
I_{2} \leq 12 s c_{2} \Delta t\left\|\mathbf{e}^{n}\right\|_{h}\left(\Delta x \Delta y \sum_{i=1}^{N_{1}} \sum_{j=1}^{N_{2}}\left(\nabla_{1, h}^{-} e_{i, j}^{n+1}\right)^{2}\right)^{1 / 2}
$$

In a similar way, one obtains

$$
I_{3} \leq 12 s c_{3} \Delta t\left\|\mathbf{e}^{n}\right\|_{h}\left(\Delta x \Delta y \sum_{i=1}^{N_{1}} \sum_{j=1}^{N_{2}}\left(\nabla_{2, h}^{-} e_{i, j}^{n+1}\right)^{2}\right)^{1 / 2} .
$$

Combining (3.26) and (3.27), and applying Young's inequality, we obtain

$$
I_{2}+I_{3} \leq 6 \Delta t\left(\frac{2 s^{2} c_{4}^{2}}{\delta_{1}}\left\|\mathbf{e}^{n}\right\|_{h}^{2}+\delta_{1}\left|\mathbf{e}^{n+1}\right|_{1, h}^{2}\right),
$$

where $c_{4}=\max \left\{c_{2}, c_{3}\right\}$.

$$
\begin{aligned}
I_{4} & \leq 3 c_{2} \Delta t\left(\Delta x \Delta y \sum_{i=1}^{N_{1}} \sum_{j=1}^{N_{2}}\left[\left(e_{i, j}^{n}\right)^{2}+\left(e_{i-1, j}^{n}\right)^{2}\right]\left|\nabla_{1, h}^{-} e_{i, j}^{n+1}\right|\right) \\
& \leq 6 c_{2} \Delta t\left\|\mathbf{e}^{n}\right\|_{\infty, h}\left\|\mathbf{e}^{n}\right\|_{h}\left(\Delta x \Delta y \sum_{i=1}^{N_{1}} \sum_{j=1}^{N_{2}}\left(\nabla_{1, h}^{-} e_{i, j}^{n+1}\right)^{2}\right)^{1 / 2} .
\end{aligned}
$$


Similarly, we get

$$
I_{5} \leq 6 c_{3} \Delta t\left\|\mathbf{e}^{n}\right\|_{\infty, h}\left\|\mathbf{e}^{n}\right\|_{h}\left(\Delta x \Delta y \sum_{i=1}^{N_{1}} \sum_{j=1}^{N_{2}}\left(\nabla_{2, h}^{-} e_{i, j}^{n+1}\right)^{2}\right)^{1 / 2} .
$$

Adding $(3.29)$ and 3.30$)$, one obtains

$$
\begin{aligned}
I_{4}+I_{5} & \leq 6 \sqrt{2} c_{4} \Delta t\left\|\mathbf{e}^{n}\right\|_{\infty, h}\left\|\mathbf{e}^{n}\right\|_{h}\left|\mathbf{e}^{n+1}\right|_{1, h} \\
& \leq 3 \Delta t\left(\frac{2 c_{4}^{2}}{\delta_{2}}\left\|\mathbf{e}^{n}\right\|_{h}^{2}+\delta_{2}\left\|\mathbf{e}^{n}\right\|_{\infty, h}^{2}\left|\mathbf{e}^{n+1}\right|_{1, h}^{2}\right) .
\end{aligned}
$$

From the definition of the bilinear map $C_{h}$, we have

$$
\begin{aligned}
& \left(C_{h}\left(\boldsymbol{v}^{n+1}, \tilde{\mathbf{e}}^{n}\right), \mathbf{e}^{n+1}\right)_{h} \\
& =\Delta x \Delta y \alpha \sum_{i=1}^{N_{1}} \sum_{j=1}^{N_{2}}\left[v_{i, j}^{n+1}\left(\boldsymbol{\beta} \cdot \nabla_{h}^{+}\right) \tilde{e}_{i, j}^{n}+\tilde{e}_{i, j}^{n}\left(\boldsymbol{\beta} \cdot \nabla_{h}^{-}\right) v_{i, j}^{n+1}+\tilde{e}_{i+1, j}^{n} \nabla_{1, h}^{+} v_{i, j}^{n+1}+\tilde{e}_{i, j+1}^{n} \nabla_{2, h}^{+} v_{i, j}^{n+1}\right] e_{i, j}^{n+1} \\
& +\Delta x \Delta y \alpha \sum_{i=1}^{N_{1}} \sum_{j=1}^{N_{2}}\left[v_{i, j}^{n+1}\left(\boldsymbol{\beta} \cdot \nabla_{h}^{-}\right) \tilde{e}_{i, j}^{n}+\tilde{e}_{i, j}^{n}\left(\boldsymbol{\beta} \cdot \nabla_{h}^{+}\right) v_{i, j}^{n+1}+\tilde{e}_{i-1, j}^{n} \nabla_{1, h}^{-} v_{i, j}^{n+1}+\tilde{e}_{i, j-1}^{n} \nabla_{2, h}^{-} v_{i, j}^{n+1}\right] e_{i, j}^{n+1} .
\end{aligned}
$$

We now estimate each of the terms in 3.32 .

$$
\begin{aligned}
\Delta x \Delta y \sum_{i=1}^{N_{1}} \sum_{j=1}^{N_{2}} v_{i, j}^{n+1}\left(\boldsymbol{\beta} \cdot \nabla_{h}^{-} \tilde{e}_{i, j}^{n}\right) e_{i, j}^{n+1} & =-\Delta x \Delta y \sum_{i=1}^{N_{1}} \sum_{j=1}^{N_{2}} \tilde{e}_{i, j}^{n}\left(\beta \cdot \nabla_{h}^{+}\right)\left(v_{i, j}^{n+1} e_{i, j}^{n+1}\right) \\
& \leq\left(c_{2}+c_{3}\right)\left\|\tilde{\mathbf{e}}^{n}\right\|_{h}\left\|\mathbf{e}^{n+1}\right\|_{h}+\sqrt{2} s\left\|\tilde{\mathbf{e}}^{n}\right\|_{h}\left|\mathbf{e}^{n+1}\right|_{1, h} \\
& =c_{5}\left\|\tilde{\mathbf{e}}^{n}\right\|_{h}\left\|\mathbf{e}^{n+1}\right\|_{h}+\sqrt{2} s\left\|\tilde{\mathbf{e}}^{n}\right\|_{h}\left|\mathbf{e}^{n+1}\right|_{1, h},
\end{aligned}
$$

where $c_{5}=c_{2}+c_{3}$.

$$
\begin{aligned}
& \Delta x \Delta y \sum_{i=1}^{N_{1}} \sum_{j=1}^{N_{2}} \tilde{e}_{i, j}^{n}\left(\boldsymbol{\beta} \cdot \nabla_{h}^{-} v_{i, j}^{n+1}\right) e_{i, j}^{n+1} \leq \max _{\substack{1 \leq i \leq N_{1} \\
1 \leq j \leq N_{2}}}\left|\left(\boldsymbol{\beta} \cdot \nabla_{h}^{-}\right) v_{i, j}^{n+1}\right|\left[\Delta x \Delta y \sum_{i=1}^{N_{1}} \sum_{j=1}^{N_{2}}\left|\tilde{e}_{i, j}^{n}\right|\left|e_{i, j}^{n+1}\right|\right] \\
& \leq\left[\max _{\substack{1 \leq i \leq N_{1} \\
1 \leq j \leq N_{2}}}\left|\nabla_{1, h}^{-} v_{i, j}^{n+1}\right|+\max _{\substack{1 \leq i \leq N_{1} \\
1 \leq j \leq N_{2}}}\left|\nabla_{2, h}^{-} v_{i, j}^{n+1}\right|\right]\left[\Delta x \Delta y \sum_{i=1}^{N_{1}} \sum_{j=1}^{N_{2}}\left|\tilde{e}_{i, j}^{n}\right|\left|e_{i, j}^{n+1}\right|\right] \\
& \leq c_{5}\left\|\tilde{\mathbf{e}}^{n}\right\|_{h}\left\|\mathbf{e}^{n+1}\right\|_{h} . \\
& +\max _{\substack{1 \leq i \leq N_{1} \\
1 \leq j \leq N_{2}}}\left|\nabla_{2, h}^{+} v_{i, j}^{n+1}\right|\left[\Delta x \Delta y \sum_{i=1}^{N_{1}} \sum_{j=1}^{N_{2}}\left|\tilde{e}_{i, j+1}^{n}\right|\left|e_{i, j}^{n+1}\right|\right] \\
& \leq c_{2}\left\|\tilde{\mathbf{e}}^{n}\right\|_{h}\left\|\mathbf{e}^{n+1}\right\|_{h}+c_{3}\left\|\tilde{\mathbf{e}}^{n}\right\|_{h}\left\|\mathbf{e}^{n+1}\right\|_{h} \\
& =c_{5}\left\|\tilde{\mathbf{e}}^{n}\right\|_{h}\left\|\mathbf{e}^{n+1}\right\|_{h} \text {. }
\end{aligned}
$$


In a similar fashion, we obtain

$$
\begin{aligned}
& \Delta x \Delta y \sum_{i=1}^{N_{1}} \sum_{j=1}^{N_{2}} v_{i, j}^{n+1}\left(\beta \cdot \nabla_{h}^{-} \tilde{e}_{i, j}^{n}\right) e_{i, j}^{n+1} \leq c_{5}\left\|\tilde{\mathbf{e}}^{n}\right\|_{h}\left\|\mathbf{e}^{n+1}\right\|_{h}+\sqrt{2} s\left\|\tilde{\mathbf{e}}^{n}\right\|_{h}\left|\mathbf{e}^{n+1}\right|_{1, h} . \\
& \Delta x \Delta y \sum_{i=1}^{N_{1}} \sum_{j=1}^{N_{2}} \tilde{e}_{i, j}^{n}\left(\beta \cdot \nabla_{h}^{+} v_{i, j}^{n+1}\right) e_{i, j}^{n+1} \leq c_{5}\left\|\tilde{\mathbf{e}}^{n}\right\|_{h}\left\|\mathbf{e}^{n+1}\right\|_{h} . \\
& \Delta x \Delta y \sum_{i=1}^{N_{1}} \sum_{j=1}^{N_{2}}\left[\tilde{e}_{i-1, j}^{n} \nabla_{1, h}^{-} v_{i, j}^{n+1}+\tilde{e}_{i, j-1}^{n} \nabla_{2, h}^{-} v_{i, j}^{n+1}\right] e_{i, j}^{n+1} \leq c_{5}\left\|\tilde{\mathbf{e}}^{n}\right\|_{h}\left\|\mathbf{e}^{n+1}\right\|_{h} .
\end{aligned}
$$

Combining the inequalities from 3.33-3.38, and using Young's inequality, we obtain

$$
2 \Delta t\left(C_{h}\left(\boldsymbol{v}^{n+1}, \tilde{\mathbf{e}}^{n}\right), \mathbf{e}^{n+1}\right)_{h} \leq \Delta t\left[\frac{8 s^{2} \alpha^{2}}{\delta_{4}}\left\|\tilde{\mathbf{e}}^{n}\right\|_{h}^{2}+\delta_{4}\left|\mathbf{e}^{n+1}\right|_{1, h}^{2}\right]+\Delta t\left[\frac{36 c_{5}^{2} \alpha^{2}}{\delta_{5}}\left\|\tilde{\mathbf{e}}^{n}\right\|_{h}^{2}+\delta_{5}\left\|\mathbf{e}^{n+1}\right\|_{h}^{2}\right]
$$

Finally, using 3.22 and Young's inequality, we obtain

$$
\left(\mathbf{r}^{n}, \mathbf{e}^{n+1}\right)_{h}=\Delta x \Delta y \sum_{i=1}^{N_{1}} \sum_{j=1}^{N_{2}} r_{i, j}^{n} e_{i, j}^{n+1} \leq \frac{16 L_{1}^{2} L_{2}^{2} c_{1}^{2}}{2 \delta_{3}}\left(\Delta t+\Delta x^{2}+\Delta y^{2}\right)^{2}+\frac{\delta_{3}}{2}\left\|\mathbf{e}^{n+1}\right\|_{h}^{2} .
$$

Thus, from 3.28, 3.31, 3.39 and 3.40, we get

$$
\begin{aligned}
\left\|\mathbf{e}^{n+1}\right\|_{h}^{2}-\left\|\mathbf{e}^{n}\right\|_{h}^{2} & +2 \Delta t \varepsilon^{2}\left|\mathbf{e}^{n+1}\right|_{2, h}^{2} \leq 2 \Delta t\left|\mathbf{e}^{n+1}\right|_{1, h}^{2}+6 \Delta t\left(\frac{2 s^{2} c_{4}^{2}}{\delta_{1}}\left\|\mathbf{e}^{n}\right\|_{h}^{2}+\delta_{1}\left|\mathbf{e}^{n+1}\right|_{1, h}^{2}\right) \\
& +3 \Delta t\left(\frac{2 c_{4}^{2}}{\delta_{2}}\left\|\mathbf{e}^{n}\right\|_{h}^{2}+\delta_{2}\left\|\mathbf{e}^{n}\right\|_{\infty, h}^{2}\left|\mathbf{e}^{n+1}\right|_{1, h}^{2}\right)+\Delta t\left[\frac{8 s^{2} \alpha^{2}}{\delta_{4}}\left\|\tilde{\mathbf{e}}^{n}\right\|_{h}^{2}+\delta_{4}\left|\mathbf{e}^{n+1}\right|_{1, h}^{2}\right] \\
& +\Delta t\left[\frac{36 c_{5}^{2} \alpha^{2}}{\delta_{5}}\left\|\tilde{\mathbf{e}}^{n}\right\|_{h}^{2}+\delta_{5}\left\|\mathbf{e}^{n+1}\right\|_{h}^{2}\right]+\frac{16 \Delta t L_{1}^{2} L_{2}^{2} c_{1}^{2}}{\delta_{3}}\left(\Delta t+\Delta x^{2}+\Delta y^{2}\right)^{2}+\Delta t \delta_{3}\left\|\mathbf{e}^{n+1}\right\|_{h}^{2},
\end{aligned}
$$

which after using 2.13 and applying Lemma 2.5 and Young's inequality gives

$$
\begin{aligned}
{\left[1-\Delta t\left(\delta_{3}+\delta_{5}\right)\right]\left\|\mathbf{e}^{n+1}\right\|_{h}^{2}+\Delta t \varepsilon^{2}\left|\mathbf{e}^{n+1}\right|_{2, h}^{2} \leq \frac{\Delta t\left(2+6 \delta_{1}+\delta_{4}\right)^{2}}{4 \varepsilon^{2}}\left\|\mathbf{e}^{n+1}\right\|_{h}^{2}+\left[1+6 \Delta t\left(\frac{2 s^{2} c_{4}^{2}}{\delta_{1}}+\frac{c_{4}^{2}}{\delta_{2}}\right)\right]\left\|\mathbf{e}^{n}\right\|_{h}^{2} } \\
+\frac{3 \Delta t \delta_{2}}{\Delta x \Delta y}\left\|\mathbf{e}^{n}\right\|_{h}^{2}\left|\mathbf{e}^{n+1}\right|_{1, h}^{2}+4 \Delta t \alpha^{2}\left[\frac{2 s^{2}}{\delta_{4}}+\frac{9 c_{5}^{2}}{\delta_{5}}\right]\left\|\tilde{\mathbf{e}}^{n}\right\|_{h}^{2}+\frac{16 \Delta t L_{1}^{2} L_{2}^{2} c_{1}^{2}}{\delta_{3}}\left(\Delta t+\Delta x^{2}+\Delta y^{2}\right)^{2} .
\end{aligned}
$$

Using 2.24, 3.41 gives

$$
\begin{gathered}
{[1-\Delta t c]\left\|\mathbf{e}^{n+1}\right\|_{h}^{2}+\Delta t \varepsilon^{2} \eta^{2}\left|\mathbf{e}^{n+1}\right|_{1, h}^{2} \leq\left(1+\Delta t c_{9}\right)\left\|\mathbf{e}^{n}\right\|_{h}^{2}+\Delta t c_{8}\left\|\tilde{\mathbf{e}}^{n}\right\|_{h}^{2}+\frac{12 \Delta t \delta_{2}}{\Delta x \Delta y}\left\|\mathbf{e}^{n}\right\|_{h}^{2}\left|\mathbf{e}^{n+1}\right|_{1, h}^{2}} \\
+\Delta t c_{7}\left(\Delta t+\Delta x^{2}+\Delta y^{2}\right)^{2}
\end{gathered}
$$

where

$$
c_{7}=\frac{16 L_{1}^{2} L_{2}^{2} c_{1}^{2}}{\delta_{3}}, \quad c=\delta_{3}+\delta_{5}+\frac{\left(2+6 \delta_{1}+\delta_{4}\right)^{2}}{4 \varepsilon^{2}} \quad c_{9}=6\left(\frac{2 s^{2} c_{4}^{2}}{\delta_{1}}+\frac{c_{4}^{2}}{\delta_{2}}\right), \quad c_{8}=4 \alpha^{2}\left[\frac{2 s^{2}}{\delta_{4}}+\frac{9 c_{5}^{2}}{\delta_{5}}\right]
$$

For

$$
\Delta t \leq \frac{1}{2 c} \equiv c
$$


then it follows from 3.42 that

$$
\begin{gathered}
\left\|\mathbf{e}^{n+1}\right\|_{h}^{2}+\Delta t \varepsilon^{2} \eta^{2}\left|\mathbf{e}^{n+1}\right|_{1, h}^{2} \leq 4^{\Delta t c}\left[\left(1+\Delta t c_{9}\right)\left\|\mathbf{e}^{n}\right\|_{h}^{2}+\Delta t c_{8}\left\|\tilde{\mathbf{e}}^{n}\right\|_{h}^{2}+\frac{3 \Delta t \delta_{2}}{\Delta x \Delta y}\left\|\mathbf{e}^{n}\right\|_{h}^{2}\left|\mathbf{e}^{n+1}\right|_{1, h}^{2}\right. \\
\left.+\Delta t c_{7}\left(\Delta t+\Delta x^{2}+\Delta y^{2}\right)^{2}\right] .
\end{gathered}
$$

Now for

$$
\frac{3 \delta_{2} c_{7}}{\Delta x \Delta y}\left[\left(\Delta t+\Delta x^{2}+\Delta y^{2}\right)^{2}\right] \leq \frac{c_{10}}{2} \varepsilon^{2} \eta^{2} 4^{-T c} \exp \left(-T c_{10}\right)
$$

where $c_{10}=c_{9}+m_{0} A c_{8}$ and $A=\sum_{i=1}^{m_{0}}\left|a_{i}\right|^{2}$, we prove by inductive method that

$$
\left\|\mathbf{e}^{n+1}\right\|_{h}^{2}+\frac{1}{2} \Delta t \varepsilon^{2} \eta^{2}\left|\mathbf{e}^{n+1}\right|_{1, h}^{2} \leq 4^{\Delta t c}\left[\left(1+\Delta t c_{9}\right)\left\|\mathbf{e}^{n}\right\|_{h}^{2}+\Delta t c_{8}\left\|\tilde{\mathbf{e}}^{n}\right\|_{h}^{2}+\Delta t c_{7}\left(\Delta t+\Delta x^{2}+\Delta y^{2}\right)^{2}\right] .
$$

For $n=0$ from 3.44, one obtains

$\left\|\mathbf{e}^{1}\right\|_{h}^{2}+\Delta t \varepsilon^{2} \eta^{2}\left|\mathbf{e}^{1}\right|_{1, h}^{2} \leq 4^{\Delta t c}\left[\left(1+\Delta t\left(c_{9}+c_{8}\right)\right)\left\|\mathbf{e}^{0}\right\|_{h}^{2}+\frac{3 \Delta t \delta_{2}}{\Delta x \Delta y}\left\|\mathbf{e}^{0}\right\|_{h}^{2}\left|\mathbf{e}^{1}\right|_{1, h}^{2}\right]+\Delta t c_{7}\left(4^{\Delta t c}\right)\left[\left(\Delta t+\Delta x^{2}+\Delta y^{2}\right)^{2}\right]$,

and hence,

$$
\left\|\mathbf{e}^{1}\right\|_{h}^{2}+\frac{1}{2} \Delta t \varepsilon^{2} \eta^{2}\left|\mathbf{e}^{1}\right|_{1, h}^{2} \leq 4^{\Delta t c}\left[\left(1+\Delta t\left(c_{9}+c_{8}\right)\right)\left\|\mathbf{e}^{0}\right\|_{h}^{2}+\Delta t c_{7}\left(\Delta t+\Delta x^{2}+\Delta y^{2}\right)^{2}\right],
$$

which is 3.46 for $n=0$. Now suppose that 3.46 is true up to the order $n-1$. Thus, for $s=0,1, \ldots, n-1$,

$$
\left\|\mathbf{e}^{s+1}\right\|_{h}^{2}+\frac{1}{2} \Delta t \varepsilon^{2} \eta^{2}\left|\mathbf{e}^{s+1}\right|_{1, h}^{2} \leq 4^{\Delta t c}\left[\left(1+\Delta t c_{9}\right)\left\|\mathbf{e}^{s}\right\|_{h}^{2}+\Delta t c_{8}\left\|\tilde{\mathbf{e}}^{s}\right\|_{h}^{2}+\Delta t c_{7}\left(\Delta t+\Delta x^{2}+\Delta y^{2}\right)^{2}\right] .
$$

It is now remaining to treat the term $\left\|\tilde{\mathbf{e}}^{s}\right\|_{h}^{2}$. For $s<m_{0}-1$, it is clear from (3.3) that $\left\|\tilde{\mathbf{e}}^{s}\right\|_{h}=\left\|\mathbf{e}^{s}\right\|_{h}$. Thus, (3.47) becomes

$$
\left\|\mathbf{e}^{s+1}\right\|_{h}^{2}+\frac{1}{2} \Delta t \varepsilon^{2} \eta^{2}\left|\mathbf{e}^{s+1}\right|_{1, h}^{2} \leq 4^{\Delta t c}\left[\left(1+\Delta t\left(c_{9}+c_{8}\right)\right)\left\|\mathbf{e}^{s}\right\|_{h}^{2}+\Delta t c_{7}\left(\Delta t+\Delta x^{2}+\Delta y^{2}\right)^{2}\right] .
$$

It follows from 3.48 that

$$
\left\|\mathbf{e}^{s+1}\right\|_{h}^{2} \leq 4^{\Delta t c}\left[\left(1+\Delta t\left(c_{9}+c_{8}\right)\right)\left\|\mathbf{e}^{s}\right\|_{h}^{2}+\Delta t c_{7}\left(\Delta t+\Delta x^{2}+\Delta y^{2}\right)^{2}\right] .
$$

After $s+1$ iterations, we get

$$
\left\|\mathbf{e}^{s+1}\right\|_{h}^{2} \leq 4^{\Delta t(s+1) c}\left[\Delta t c_{7}\left(\Delta t+\Delta x^{2}+\Delta y^{2}\right)^{2} \sum_{j=0}^{s}\left(1+\Delta t\left(c_{9}+c_{8}\right)\right)^{j}\right] .
$$

For the case $s \geq m_{0}-1$ and $m_{0}>1$, it follows from 3.2 that

$$
\left\|\tilde{\mathbf{e}}^{s}\right\|_{h} \leq \sum_{i=1}^{m_{0}}\left|a_{i}\right|\left\|\tilde{\mathbf{e}}^{s-i+1}\right\|_{h}
$$

which by Cauchy-Schwartz's inequality gives

$$
\left\|\tilde{\mathbf{e}}^{s}\right\|_{h}^{2} \leq A\left[\left\|\mathbf{e}^{s}\right\|_{h}^{2}+\left\|\mathbf{e}^{s-1}\right\|_{h}^{2}+\cdots+\left\|\mathbf{e}^{s-m_{0}+1}\right\|_{h}^{2}\right] .
$$


Hence, 3.47 gives

$$
\begin{aligned}
\left\|\mathbf{e}^{s+1}\right\|_{h}^{2} & \leq 4^{\Delta t c}\left[\left(1+\Delta t c_{9}\right)\left\|\mathbf{e}^{s}\right\|_{h}^{2}+\Delta t c_{8} A\left(\left\|\mathbf{e}^{s}\right\|_{h}^{2}+\left\|\mathbf{e}^{s-1}\right\|_{h}^{2}+\cdots+\left\|\mathbf{e}^{s-m_{0}+1}\right\|_{h}^{2}\right)\right] \\
& +4^{\Delta t c}\left[\Delta t c_{7}\left(\Delta t+\Delta x^{2}+\Delta y^{2}\right)^{2}\right] .
\end{aligned}
$$

It follows easily that

$$
\begin{gathered}
\max \left\{\left\|\mathbf{e}^{s+1}\right\|_{h}^{2},\left\|\mathbf{e}^{s}\right\|_{h}^{2}, \ldots,\left\|\mathbf{e}^{s-m_{0}+2}\right\|_{h}^{2}\right\} \leq 4^{\Delta t c}\left[\left(1+\Delta t c_{10}\right) \max \left\{\left\|\mathbf{e}^{s}\right\|_{h}^{2},\left\|\mathbf{e}^{s-1}\right\|_{h}^{2}, \ldots,\left\|\mathbf{e}^{s-m_{0}+1}\right\|_{h}^{2}\right\}\right. \\
\left.+\Delta t c_{7}\left(\Delta t+\Delta x^{2}+\Delta y^{2}\right)^{2}\right]
\end{gathered}
$$

which after $s-m_{0}+2$ iterations gives

$$
\begin{aligned}
\max \left\{\left\|\mathbf{e}^{s+1}\right\|_{h}^{2},\left\|\mathbf{e}^{s}\right\|_{h}^{2}, \ldots,\left\|\mathbf{e}^{s-m_{0}+2}\right\|_{h}^{2}\right\} & \\
\leq 4^{\left(s-m_{0}+2\right) \Delta t c}[ & \left(1+\Delta t c_{10}\right)^{s-m_{0}+2} \max \left\{\left\|\mathbf{e}^{m_{0}-1}\right\|_{h}^{2},\left\|\mathbf{e}^{m_{0}-2}\right\|_{h}^{2}, \ldots,\left\|\mathbf{e}^{0}\right\|_{h}^{2}\right\} \\
& \left.\quad+\Delta t c_{7}\left(\Delta t+\Delta x^{2}+\Delta y^{2}\right)^{2} \sum_{j=0}^{s-m_{0}+1}\left(1+\Delta t c_{10}\right)^{j}\right] .
\end{aligned}
$$

Combining (3.49) and 3.53 , we get

$$
\begin{aligned}
\left\|\mathbf{e}^{s+1}\right\|_{h}^{2} & \leq 4^{(s+1) \Delta t c}\left[\Delta t c_{7}\left(\Delta t+\Delta x^{2}+\Delta y^{2}\right)^{2} \sum_{j=0}^{s}\left(1+\Delta t c_{10}\right)^{j}\right] \\
& =4^{(s+1) \Delta t c}\left[\Delta t c_{7}\left(\Delta t+\Delta x^{2}+\Delta y^{2}\right)^{2}\left(\frac{\left(1+\Delta t c_{10}\right)^{s+1}-1}{\Delta t c_{10}}\right)\right] \\
& \leq 4^{(s+1) \Delta t c} \exp \left((s+1) \Delta t c_{10}\right)\left[\frac{c_{7}}{c_{10}}\left(\Delta t+\Delta x^{2}+\Delta y^{2}\right)^{2}\right]
\end{aligned}
$$

and

$$
\left\|\tilde{\mathbf{e}}^{s+1}\right\|_{h}^{2} \leq m_{0} A 4^{(s+1) \Delta t c} \exp \left((s+1) \Delta t c_{10}\right)\left[\frac{c_{7}}{c_{10}}\left(\Delta t+\Delta x^{2}+\Delta y^{2}\right)^{2}\right]
$$

for $s=0,1, \ldots, n-1$.

Going back to (3.44), and using (3.54) we obtain

$$
\begin{aligned}
\left\|\mathbf{e}^{n+1}\right\|_{h}^{2}+\Delta t \varepsilon^{2} \eta^{2}\left|\mathbf{e}^{n+1}\right|_{1, h}^{2} \leq & 4 t c\left[\left(1+\Delta t c_{9}\right)\left\|\mathbf{e}^{n}\right\|_{h}^{2}+\Delta t c_{8}\left|\tilde{\mathbf{e}}^{n}\right|_{h}^{2}+\Delta t c_{7}\left(\Delta t+\Delta x^{2}+\Delta y^{2}\right)^{2}\right] \\
& +\frac{3 \Delta t \delta_{2}}{\Delta x \Delta y} 4^{n \Delta t c} \exp \left(n \Delta t c_{10}\right)\left[\frac{c_{7}}{c_{10}}\left(\Delta t+\Delta x^{2}+\Delta y^{2}\right)^{2}\right]\left|\mathbf{e}^{n+1}\right|_{1, h}^{2},
\end{aligned}
$$

which by 3.45 gives

$\left\|\mathbf{e}^{n+1}\right\|_{h}^{2}+\Delta t \varepsilon^{2} \eta^{2}\left|\mathbf{e}^{n+1}\right|_{1, h}^{2} \leq 4^{\Delta t c}\left[\left(1+\Delta t c_{9}\right)\left\|\mathbf{e}^{n}\right\|_{h}^{2}+\Delta t c_{8}\left\|\tilde{\mathbf{e}}^{n}\right\|_{h}^{2}+\Delta t c_{7}\left(\Delta t+\Delta x^{2}+\Delta y^{2}\right)^{2}\right]+\frac{1}{2} \Delta t \varepsilon^{2} \eta^{2}\left|\mathbf{e}^{n+1}\right|_{1, h}^{2}$.

Thus, we have

$$
\left\|\mathbf{e}^{n+1}\right\|_{h}^{2}+\frac{1}{2} \Delta t \varepsilon^{2} \eta^{2}\left|\mathbf{e}^{n+1}\right|_{1, h}^{2} \leq 4^{\Delta t c}\left[\left(1+\Delta t c_{9}\right)\left\|\mathbf{e}^{n}\right\|_{h}^{2}+\Delta t c_{8}\left\|\tilde{\mathbf{e}}^{n}\right\|_{h}^{2}+\Delta t c_{7}\left(\Delta t+\Delta x^{2}+\Delta y^{2}\right)^{2}\right]
$$


Using (3.54) and 3.55), 3.56) gives

$$
\begin{aligned}
\left\|\mathbf{e}^{n}\right\|_{h}^{2} & \leq 4^{n \Delta t c} \exp \left(n \Delta t c_{10}\right)\left[\frac{c_{7}}{c_{10}}\left(\Delta t+\Delta x^{2}+\Delta y^{2}\right)^{2}\right] \\
& \leq 4^{T c} \exp \left(T c_{10}\right)\left[\frac{c_{7}}{c_{10}}\left(\Delta t+\Delta x^{2}+\Delta y^{2}\right)^{2}\right],
\end{aligned}
$$

for $n=1,2, \ldots, M$. Thus, it follows from (3.57) that

$$
\left\|\mathbf{e}^{n}\right\|_{h} \leq C\left(\Delta t+\Delta x^{2}+\Delta y^{2}\right)
$$

for constant $C$ independent of $\Delta t, \Delta x$ and $\Delta y$. This completes the proof.

Remark 3.3. The assumption $\Delta t<4 \varepsilon^{2}$ in Theorem 3.3 is to ensure the existence of solution for (3.5a)(3.5c). It is worth noting that the convergence result Theorem 3.3 is conducted for $\alpha_{1}=\alpha_{2}$. The case when $\alpha_{1} \neq \alpha_{2}$ is treated in the same way but the rate of convergence will change.

\subsection{Explicit Finite Volume Method}

In this section, we approximate the solution of 1.1) using an explicit finite volume method:

$$
\begin{aligned}
& \frac{u_{i, j}^{n+1}-u_{i, j}^{n}}{\Delta t}-\left(C_{h}\left(\mathbf{u}^{n}, \mathbf{u}^{n}\right)\right)_{i, j}+\varepsilon^{2} \Delta_{h}^{2} u_{i, j}^{n}=\nabla_{1, h}^{+}\left(\varphi_{i-1 / 2, j}^{n} \nabla_{1, h}^{-} u_{i, j}^{n}\right)+\nabla_{2, h}^{+}\left(\varphi_{i, j-1 / 2}^{n} \nabla_{2, h}^{-} u_{i, j}^{n}\right), \\
& u_{i, j}^{n}=u_{i+N_{1}, j}^{n}=u_{i, j+N_{2}}^{n}, \\
& u_{i, j}^{0}=\frac{1}{\Delta x \Delta y} \iint_{k_{i, j}} u^{0}(x, y) d x d y,
\end{aligned}
$$

for $1 \leq n \leq M-1$ and $1 \leq i \leq N_{1}, 1 \leq j \leq N_{2}$. (3.58) is explicit, hence the solution $\mathbf{u}^{n}$ is computed at each time step. One important feature of this scheme is stated in the following.

Theorem 3.4. We assume that the following are satisfied for some $\delta, 0<\delta<1$ :

$$
\begin{aligned}
& \Delta t\left(\frac{1}{\Delta x^{2}}+\frac{1}{\Delta y^{2}}\right)^{2} \leq \frac{1-\delta}{64 \varepsilon^{2}}, \\
& 16 \Delta t\left(\frac{1}{\Delta x^{2}}+\frac{1}{\Delta y^{2}}\right) \leq \varepsilon^{2} \delta \eta^{2}(1-\delta), \\
& \frac{72 \Delta t}{\Delta x \Delta y}\left(\left(\left|\alpha_{1}\right|+\left|\alpha_{2}\right|\right)^{2}+\frac{4}{\Delta x \Delta y}\left(\frac{1}{\Delta x^{2}}+\frac{1}{\Delta y^{2}}\right)\left\|\boldsymbol{u}^{0}\right\|_{h}^{2}\right)\left\|\boldsymbol{u}^{0}\right\|_{h}^{2} \leq \varepsilon^{2} \delta^{2} \eta^{2} \exp \left(\frac{-2 T}{\varepsilon^{2}}\right),
\end{aligned}
$$

where $\alpha_{1}$ and $\alpha_{2}$ are the constants in 2.20). Then the finite volume method defined by 3.58 is $L^{\infty}\left(0, T ; \mathcal{H}_{h}\right)$ stable in the following sense:

$$
\begin{aligned}
& \left\|\boldsymbol{u}^{n}\right\|^{2} \leq \exp \left(\frac{\Delta t}{\varepsilon^{2}}\right)\left\|\boldsymbol{u}^{n-1}\right\|^{2} \leq \cdots \leq \exp \left(\frac{n \Delta t}{\varepsilon^{2}}\right)\left\|\boldsymbol{u}^{0}\right\|^{2} \leq \exp \left(\frac{T}{\varepsilon^{2}}\right)\left\|\boldsymbol{u}^{0}\right\|^{2}, n=1,2 \ldots, M, \\
& \frac{\Delta t}{2} \varepsilon^{2} \delta^{2} \eta^{2} \sum_{n=0}^{M-1} \exp \left(\frac{(M-n) \Delta t}{\varepsilon^{2}}\right)\left|\boldsymbol{u}^{n}\right|_{1, h}^{2} \leq \exp \left(\frac{T}{\varepsilon^{2}}\right)\left\|\boldsymbol{u}^{0}\right\|^{2}
\end{aligned}
$$

Proof. To prove this assertion we use the approach of Temam [31. Multiplying (3.58a) by $2 \Delta t \Delta x \Delta y u_{i, j}^{n}$ and summing the equalities for $i=1, \ldots, N_{1}$ and $j=1, \ldots, N_{2}$, together with 2.16), 2.18) and Lemma 2.5 . we arrive at

$$
\left\|\mathbf{u}^{n+1}\right\|_{h}^{2}-\left\|\mathbf{u}^{n+1}-\mathbf{u}^{n}\right\|_{h}^{2}+\Delta t \varepsilon^{2}\left|\mathbf{u}^{n}\right|_{2, h}^{2} \leq \exp \left(\frac{\Delta t}{\varepsilon^{2}}\right)\left\|\mathbf{u}^{n}\right\|_{h}^{2}
$$


Now we have to estimate the term $\left\|\mathbf{u}^{n+1}-\mathbf{u}^{n}\right\|_{h}^{2}$. By multiplying 3.58a by $2 \Delta t \Delta x \Delta y\left(u_{i, j}^{n+1}-u_{i, j}^{n}\right)$ and adding the corresponding equalities for $i=1, \ldots, N_{1}$ and $j=1, \ldots, N_{2}$, we obtain

$$
\begin{aligned}
2\left\|\mathbf{u}^{n+1}-\mathbf{u}^{n}\right\|_{h}^{2}= & 2 \Delta t\left(C_{h}\left(\mathbf{u}^{n}, \mathbf{u}^{n}\right), \mathbf{u}^{n+1}-\mathbf{u}^{n}\right)_{h}-2 \Delta t \varepsilon^{2}\left(\Delta_{h} \mathbf{u}^{n}, \Delta_{h}\left(\mathbf{u}^{n+1}-\mathbf{u}^{n}\right)\right) \\
& -2 \Delta t \Delta x \Delta y \sum_{i=1}^{N_{1}} \sum_{i=1}^{N_{2}}\left[\varphi_{i-1 / 2, j}^{n} \nabla_{1, h}^{-} u_{i, j}^{n+1}\right] \nabla_{1, h}^{-}\left(u_{i, j}^{n+1}-u_{i, j}^{n}\right) \\
& -2 \Delta t \Delta x \Delta y \sum_{i=1}^{N_{1}} \sum_{i=1}^{N_{2}}\left[\varphi_{i, j-1 / 2}^{n} \nabla_{2, h}^{-} u_{i, j}^{n+1}\right] \nabla_{2, h}^{-}\left(u_{i, j}^{n+1}-u_{i, j}^{n}\right)
\end{aligned}
$$

Using (2.18) and (2.19), we majorize all the terms on the right hand side of $(3.63)$ as follows:

$$
\begin{aligned}
2 \Delta t\left(C_{h}\left(\mathbf{u}^{n}, \mathbf{u}^{n}\right), \mathbf{u}^{n+1}-\mathbf{u}^{n}\right)_{h} & \leq \frac{36 \Delta t^{2}}{\Delta x \Delta y}\left(\left|\alpha_{1}\right|+\left|\alpha_{2}\right|\right)^{2}\left\|\mathbf{u}^{n}\right\|_{h}^{2}\left|\mathbf{u}^{n}\right|_{1, h}^{2}+\frac{1}{4}\left\|\mathbf{u}^{n+1}-\mathbf{u}^{n}\right\|_{h}^{2} ; \\
-2 \Delta t \varepsilon^{2}\left(\Delta_{h} \mathbf{u}^{n}, \Delta_{h}\left(\mathbf{u}^{n+1}-\mathbf{u}^{n}\right)\right)_{h} & \leq 64 \varepsilon^{4} \Delta t^{2}\left(\frac{1}{\Delta x^{2}}+\frac{1}{\Delta y^{2}}\right)^{2}\left|\mathbf{u}^{n}\right|_{2, h}^{2}+\frac{1}{4}\left\|\mathbf{u}^{n+1}-\mathbf{u}^{n}\right\|_{h}^{2} ; \\
-2 \Delta t \Delta x \Delta y \sum_{i=1}^{N_{1}} \sum_{i=1}^{N_{2}}\left[\left(\varphi_{i-1 / 2, j}^{n} \nabla_{1, h}^{-} u_{i, j}^{n+1}\right) \nabla_{1, h}^{-}\left(u_{i, j}^{n+1}-u_{i, j}^{n}\right)+\left(\varphi_{i, j-1 / 2}^{n} \nabla_{2, h}^{-} u_{i, j}^{n+1}\right) \nabla_{2, h}^{-}\left(u_{i, j}^{n+1}-u_{i, j}^{n}\right)\right] & \leq \frac{144 \Delta t^{2}}{\Delta x^{2} \Delta y^{2}}\left(\frac{1}{\Delta x^{2}}+\frac{1}{\Delta y^{2}}\right)\left\|\mathbf{u}^{n}\right\|_{h}^{4}\left|\mathbf{u}^{n}\right|_{1, h}^{2}+\frac{1}{4}\left\|\mathbf{u}^{n+1}-\mathbf{u}^{n}\right\|_{h}^{2} \\
& +16 \Delta t^{2}\left(\frac{1}{\Delta x^{2}}+\frac{1}{\Delta y^{2}}\right)\left|\mathbf{u}^{n}\right|_{1, h}^{2}+\frac{1}{4}\left\|\mathbf{u}^{n+1}-\mathbf{u}^{n}\right\|_{h}^{2} .
\end{aligned}
$$

Thus using (3.64)-(3.66), 3.63) becomes

$$
\begin{aligned}
\left\|\mathbf{u}^{n+1}-\mathbf{u}^{n}\right\|_{h}^{2} \leq & \frac{36 \Delta t^{2}}{\Delta x \Delta y}\left(\left|\alpha_{1}\right|+\left|\alpha_{2}\right|\right)^{2}\left\|\mathbf{u}^{n}\right\|_{h}^{2}\left|\mathbf{u}^{n}\right|_{1, h}^{2}+64 \varepsilon^{4} \Delta t^{2}\left(\frac{1}{\Delta x^{2}}+\frac{1}{\Delta y^{2}}\right)^{2}\left|\mathbf{u}^{n}\right|_{2, h}^{2} \\
& \quad \frac{144 \Delta t^{2}}{\Delta x^{2} \Delta y^{2}}\left(\frac{1}{\Delta x^{2}}+\frac{1}{\Delta y^{2}}\right)\left\|\mathbf{u}^{n}\right\|_{h}^{4}\left|\mathbf{u}^{n}\right|_{1, h}^{2}+16 \Delta t^{2}\left(\frac{1}{\Delta x^{2}}+\frac{1}{\Delta y^{2}}\right)\left|\mathbf{u}^{n}\right|_{1, h}^{2},
\end{aligned}
$$

which by 3.59 gives

$$
\begin{aligned}
\left\|\mathbf{u}^{n+1}-\mathbf{u}^{n}\right\|_{h}^{2} \leq & \frac{36 \Delta t^{2}}{\Delta x \Delta y}\left(\left|\alpha_{1}\right|+\left|\alpha_{2}\right|\right)^{2}\left\|\mathbf{u}^{n}\right\|_{h}^{2}\left|\mathbf{u}^{n}\right|_{1, h}^{2}+\Delta t \varepsilon^{2}(1-\delta)\left|\mathbf{u}^{n}\right|_{2, h}^{2} \\
& \quad+\frac{144 \Delta t^{2}}{\Delta x^{2} \Delta y^{2}}\left(\frac{1}{\Delta x^{2}}+\frac{1}{\Delta y^{2}}\right)\left\|\mathbf{u}^{n}\right\|_{h}^{4}\left|\mathbf{u}^{n}\right|_{1, h}^{2}+16 \Delta t^{2}\left(\frac{1}{\Delta x^{2}}+\frac{1}{\Delta y^{2}}\right)\left|\mathbf{u}^{n}\right|_{1, h}^{2} .
\end{aligned}
$$

On substitution of (3.67) back to 3.62, we arrive at

$$
\begin{aligned}
\left\|\mathbf{u}^{n+1}\right\|_{h}^{2}-\exp \left(\frac{\Delta t}{\varepsilon^{2}}\right)\left\|\mathbf{u}^{n}\right\|_{h}^{2}+ & \Delta t \varepsilon^{2} \delta\left|\mathbf{u}^{n}\right|_{2, h}^{2} \leq 16 \Delta t^{2}\left(\frac{1}{\Delta x^{2}}+\frac{1}{\Delta y^{2}}\right)\left|\mathbf{u}^{n}\right|_{1, h}^{2} \\
& +\frac{36 \Delta t^{2}}{\Delta x \Delta y}\left[\left(\left|\alpha_{1}\right|+\left|\alpha_{2}\right|\right)^{2}+\frac{4}{\Delta x \Delta y}\left(\frac{1}{\Delta x^{2}}+\frac{1}{\Delta y^{2}}\right)\left\|\mathbf{u}^{n}\right\|_{h}^{2}\right]\left\|\mathbf{u}^{n}\right\|_{h}^{2}\left|\mathbf{u}^{n}\right|_{1, h}^{2} .
\end{aligned}
$$

Using (2.24) and (3.60), 3.68) gives

$$
\begin{aligned}
\left\|\mathbf{u}^{n+1}\right\|_{h}^{2}-\exp \left(\frac{\Delta t}{\varepsilon^{2}}\right)\left\|\mathbf{u}^{n}\right\|_{h}^{2} & +\Delta t \varepsilon^{2} \delta^{2} \eta^{2}\left|\mathbf{u}^{n}\right|_{1, h}^{2} \\
& \leq \frac{36 \Delta t^{2}}{\Delta x \Delta y}\left(\left(\left|\alpha_{1}\right|+\left|\alpha_{2}\right|\right)^{2}+\frac{4}{\Delta x \Delta y}\left(\frac{1}{\Delta x^{2}}+\frac{1}{\Delta y^{2}}\right)\left\|\mathbf{u}^{n}\right\|_{h}^{2}\right)\left\|\mathbf{u}^{n}\right\|_{h}^{2}\left|\mathbf{u}^{n}\right|_{1, h}^{2} .
\end{aligned}
$$


We then need to show by induction on $n$, that

$$
\left\|\mathbf{u}^{n+1}\right\|_{h}^{2}+\frac{\Delta t}{2} \varepsilon^{2} \delta^{2} \eta^{2}\left|\mathbf{u}^{n}\right|_{1, h}^{2} \leq \exp \left(\frac{\Delta t}{\varepsilon^{2}}\right)\left\|\mathbf{u}^{n}\right\|_{h}^{2} .
$$

For $n=0$, from 3.69, we obtain

$$
\begin{aligned}
\left\|\mathbf{u}^{1}\right\|_{h}^{2} & +\Delta t \varepsilon^{2} \delta^{2} \eta^{2}\left|\mathbf{u}^{0}\right|_{1, h}^{2} \\
& \leq \exp \left(\frac{\Delta t}{\varepsilon^{2}}\right)\left\|\mathbf{u}^{0}\right\|_{h}^{2}+\frac{36 \Delta t^{2}}{\Delta x \Delta y}\left[\left(\left|\alpha_{1}\right|+\left|\alpha_{2}\right|\right)^{2}+\frac{4}{\Delta x \Delta y}\left(\frac{1}{\Delta x^{2}}+\frac{1}{\Delta y^{2}}\right)\left\|\mathbf{u}^{0}\right\|_{h}^{2}\right]\left\|\mathbf{u}^{0}\right\|_{h}^{2}\left|\mathbf{u}^{0}\right|_{1, h}^{2},
\end{aligned}
$$

which with 3.61 leads to

$$
\left\|\mathbf{u}^{1}\right\|_{h}^{2}+\frac{\Delta t}{2} \varepsilon^{2} \delta^{2} \eta^{2}\left|\mathbf{u}^{0}\right|_{1, h}^{2} \leq \exp \left(\frac{\Delta t}{\varepsilon^{2}}\right)\left\|\mathbf{u}^{0}\right\|_{h}^{2},
$$

which is 3.70 for $n=0$. Assuming now that 3.70 is true up to the order $n-1$, this is to say that for $s=0,2, \ldots, n-1$, we have

$$
\left\|\mathbf{u}^{s}\right\|_{h}^{2} \leq \exp \left(\frac{\Delta t}{\varepsilon^{2}}\right)\left\|\mathbf{u}^{s-1}\right\|_{h}^{2} \text { and }\left\|\mathbf{u}^{s}\right\|_{h}^{2} \leq \exp \left(\frac{s \Delta t}{\varepsilon^{2}}\right)\left\|\mathbf{u}^{0}\right\|_{h}^{2} .
$$

Using (3.71) in 3.69), one obtains

$$
\begin{aligned}
\left\|\mathbf{u}^{n+1}\right\|_{h}^{2}+\Delta t \varepsilon^{2} \delta^{2} \eta^{2}\left|\mathbf{u}^{n}\right|_{1, h}^{2} \leq \exp \left(\frac{\Delta t}{\varepsilon^{2}}\right)\left\|\mathbf{u}^{n}\right\|_{h}^{2} \\
+\frac{36 \Delta t}{\Delta x \Delta y} \exp \left(\frac{2 n \Delta t}{\varepsilon^{2}}\right)\left[\left(\left|\alpha_{1}\right|+\left|\alpha_{2}\right|\right)^{2}+\frac{4}{\Delta x \Delta y}\left(\frac{1}{\Delta x^{2}}+\frac{1}{\Delta y^{2}}\right)\left\|\mathbf{u}^{0}\right\|_{h}^{2}\right]\left\|\mathbf{u}^{0}\right\|_{h}^{2}\left|\mathbf{u}^{n}\right|_{1, h}^{2},
\end{aligned}
$$

which by 3.61 gives

$$
\left\|\mathbf{u}^{n+1}\right\|_{h}^{2}+\Delta t \varepsilon^{2} \delta^{2} \eta^{2}\left|\mathbf{u}^{n}\right|_{1, h}^{2} \leq \exp \left(\frac{\Delta t}{\varepsilon^{2}}\right)\left\|\mathbf{u}^{n}\right\|_{h}^{2}+\frac{\Delta t}{2} \varepsilon^{2} \delta^{2} \eta^{2}\left|\mathbf{u}^{n}\right|_{1, h}^{2},
$$

re-written also as follows

$$
\left\|\mathbf{u}^{n+1}\right\|_{h}^{2}+\frac{\Delta t}{2} \varepsilon^{2} \delta^{2} \eta^{2}\left|\mathbf{u}^{n}\right|_{1, h}^{2} \leq \exp \left(\frac{\Delta t}{\varepsilon^{2}}\right)\left\|\mathbf{u}^{n}\right\|_{h}^{2}
$$

We then have

$$
\begin{aligned}
\left\|\mathbf{u}^{n+1}\right\|_{h}^{2} \leq & \exp \left(\frac{\Delta t}{\varepsilon^{2}}\right)\left\|\mathbf{u}^{n}\right\|_{h}^{2}-\frac{\Delta t}{2} \varepsilon^{2} \delta^{2} \eta^{2}\left|\mathbf{u}^{n}\right|_{1, h}^{2} \\
\leq & \exp \left(\frac{2 \Delta t}{\varepsilon^{2}}\right)\left\|\mathbf{u}^{n-1}\right\|_{h}^{2}-\frac{\Delta t}{2} \varepsilon^{2} \delta^{2} \eta^{2} \exp \left(\frac{\Delta t}{\varepsilon^{2}}\right)\left|\mathbf{u}^{n-1}\right|_{1, h}^{2}-\frac{\Delta t}{2} \varepsilon^{2} \delta^{2} \eta^{2}\left|\mathbf{u}^{n}\right|_{1, h}^{2} \\
\leq & \exp \left(\frac{3 \Delta t}{\varepsilon^{2}}\right)\left\|\mathbf{u}^{n-2}\right\|_{h}^{2}-\frac{\Delta t}{2} \varepsilon^{2} \delta^{2} \eta^{2} \exp \left(\frac{2 \Delta t}{\varepsilon^{2}}\right)\left|\mathbf{u}^{n-2}\right|_{1, h}^{2}-\frac{\Delta t}{2} \varepsilon^{2} \delta^{2} \eta^{2} \exp \left(\frac{\Delta t}{\varepsilon^{2}}\right)\left|\mathbf{u}^{n-1}\right|_{1, h}^{2} \\
& \quad-\frac{\Delta t}{2} \varepsilon^{2} \delta^{2} \eta^{2}\left|\mathbf{u}^{n}\right|_{1, h}^{2} \\
\vdots & \quad \exp \left(\frac{(n+1) \Delta t}{\varepsilon^{2}}\right)\left\|\mathbf{u}^{0}\right\|_{h}^{2}-\frac{\Delta t}{2} \varepsilon^{2} \delta^{2} \eta^{2} \sum_{s=0}^{n} \exp \left(\frac{(n-s) \Delta t}{\varepsilon^{2}}\right)\left|\mathbf{u}^{s}\right|_{1, h}^{2} .
\end{aligned}
$$

Hence we get

$$
\left\|\mathbf{u}^{n+1}\right\|_{h}^{2}+\frac{\Delta t}{2} \varepsilon^{2} \delta^{2} \eta^{2} \sum_{s=0}^{n} \exp \left(\frac{(n-s) \Delta t}{\varepsilon^{2}}\right)\left|\mathbf{u}^{s}\right|_{1, h}^{2} \leq \exp \left(\frac{(n+1) \Delta t}{\varepsilon^{2}}\right)\left\|\mathbf{u}^{0}\right\|_{h}^{2} .
$$

Therefore, the proof is complete. 


\section{Multilevel Finite Volume Approximation}

Multilevel methods were introduced to improve calculation speed in the simulation of complex physical phenomena while maintaining a good level of accuracy, see [22, 23, 24, 25, 26, 27. This section is an application of the work presented in 23, in which the shallow water equations is analyzed. Here, we are concerned with the two dimensional convective Cahn-Hilliard equation (1.1)-(1.4). We formulate in the spirit of [23] two methods approximating (1.1)-(1.4), namely: implicit multilevel finite volume method and explicit multilevel finite volume method. These new methods are next studied thoroughly and comparison by stability and CPU time with the associated one-level methods discussed in section 3.1 are established. To make this text self-contained for the reader, we recall below the multilevel finite volume approximation as described in Bousquet et. al. [23].

Here $N_{1}$ and $N_{2}$ are assumed to be divisible by 3. Let $N_{1}^{0}, N_{2}^{0}$ and $M_{0}$ be integers such that $3 N_{1}^{0}=$ $N_{1}, 3 N_{2}^{0}=N_{2}$ and $\Delta t M_{0}=T$. We discretize $\mathcal{M}$ into fine meshes and coarse meshes. The fine mesh consists of $3 N_{1}^{0} \times 3 N_{2}^{0}$ regular cells $\left(k_{i, j}\right)_{1 \leq i \leq 3 N_{1}^{0}, 1 \leq j \leq 3 N_{2}^{0}}$ of uniform area $\Delta x \Delta y$.

The coarse mesh consists of $N_{1}^{0} N_{2}^{0}$ control volumes $\left(K_{l, m}\right)_{1 \leq l \leq N_{1}^{0}, 1 \leq m \leq N_{2}^{0}}$ of uniform area $9 \Delta x \Delta y$, where

$$
K_{l, m}=\left(x_{3 l-2-1 / 2}, x_{3 m+1 / 2}\right) \times\left(y_{3 m-2-1 / 2}, y_{3 m+1 / 2}\right) .
$$

We denote the approximate solutions on the fine grid by $u_{i, j}, 1 \leq i \leq 3 N_{1}^{0}, 1 \leq j \leq 3 N_{2}^{0}$. The approximation on the coarse mesh is given by

$$
U_{l, m}=\frac{1}{9} \sum_{\alpha, \beta=0}^{2} u_{3 l-\alpha, 3 m-\beta}, 1 \leq l \leq N_{1}^{0}, 1 \leq m \leq N_{2}^{0},
$$

and the incremental unknowns are given by the relation

$$
Z_{3 l-\alpha, 3 m-\beta}=u_{3 l-\alpha, 3 m-\beta}-U_{l, m} .
$$

Let $p>1$ and $q>1$ be two fixed integers. We discretize (1.1) on the fine mesh by using time step $\Delta t / p$ and on the coarse mesh by using time step $\Delta t$. We assume that $n$ is a multiple of $q+1$ and $\left(u_{i, j}^{n}\right)_{1 \leq i \leq 3 N_{1}^{0}, 1 \leq j \leq 3 N_{2}^{0}}$ are known, where $u_{i, j}^{n}$ is an approximation of the average value of $u$ over $k_{i, j}$ at the grid $t=n \Delta t$, for $i=1, \ldots, 3 N_{1}^{0}, j=1, \ldots, 3 N_{2}^{0}$. For $r=0,1, \ldots, p$ and $s=1,2, \ldots, q+1$, we let $u_{i, j}^{n+r / p}$ be the approximate solution of the mean values over $k_{i, j}$ at time $t_{n+t / p}=n \Delta t+r \Delta t / p$ for $i=1, \ldots, 3 N_{1}^{0}, j=1, \ldots, 3 N_{2}^{0}$ and $U_{l, m}^{n+s}$ the approximate solution of the mean value on the coarse mesh $K_{l, m}$ at time $t_{n+s}=(n+s) \Delta t$ for $l=1, \ldots, N_{1}^{0}$ and $m=1, \ldots, N_{2}^{0}$.

\subsection{Implicit multilevel Finite volume Method}

For $0 \leq r \leq p-1$ and $1 \leq s \leq q$, the following multilevel scheme is used to discretize (1.1)-(1.4).

$$
\begin{aligned}
& \frac{p}{\Delta t}\left(u_{i, j}^{n+(r+1) / p}-u_{i, j}^{n+r / p}\right)-\left(C_{h}\left(\mathbf{u}^{n+(r+1) / p}, \tilde{\mathbf{u}}^{n+r / p}\right)\right)_{i, j}+\varepsilon^{2} \Delta_{h}^{2} u_{i, j}^{n+(r+1) / p} \\
& =\nabla_{1, h}^{+}\left(\varphi_{i-1 / 2, j}^{n+r / p} \nabla_{1, h}^{-} u_{i, j}^{n+(r+1) / p}\right)+\nabla_{2, h}^{+}\left(\varphi_{i, j-1 / 2}^{n+r / p} \nabla_{2, h}^{-} u_{i, j}^{n+(r+1) / p}\right), \\
& \frac{U_{l, m}^{n+s+1}-U_{l, m}^{n+s}}{\Delta t}-\left(C_{3 h}\left(\mathbf{U}^{n+s+1}, \tilde{\mathbf{U}}^{n+s}\right)\right)_{l, m}+\varepsilon^{2} \Delta_{3 h}^{2} U_{l, m}^{n+s+1} \\
& =\nabla_{1,3 h}^{+}\left(\Phi_{l-1 / 2, m}^{n+s} \nabla_{1,3 h}^{-} U_{l, m}^{n+s+1}\right)+\nabla_{2,3 h}^{+}\left(\Phi_{l, m-1 / 2}^{n+s} \nabla_{2,3 h}^{-} U_{l, m}^{n+s+1}\right), \\
& u_{i, j}^{n+(r+1) / p}=u_{i+3 N_{1}^{0}, j}^{n+(r+1) / p}=u_{i, j+3 N_{2}^{0}}^{n+(r+1) / p}, \\
& U_{l, m}^{n+s+1}=U_{l+N_{1}^{0}, m}^{n+s+1}=U_{l, m+N_{2}^{0}}^{n+s+1}, \\
& u_{i, j}^{0}=\frac{1}{\Delta x \Delta y}, \iint_{k_{i, j}} u^{0}(x, y) d x d y,
\end{aligned}
$$


where $1 \leq i \leq 3 N_{1}^{0}, 1 \leq j \leq 3 N_{2}^{0}, 1 \leq l \leq N_{1}^{0}, 1 \leq m \leq N_{2}^{0}$ and

$$
\begin{gathered}
\varphi_{i-1 / 2, j}^{n+r / p}=\frac{f^{\prime}\left(u_{i, j}^{n+r / p}\right)+f^{\prime}\left(u_{i-1, j}^{n+r / p}\right)}{2}, \quad \varphi_{i, j-1 / 2}^{n+r / p}=\frac{f^{\prime}\left(u_{i, j}^{n}\right)+f^{\prime}\left(u_{i, j-1}^{n+r / p}\right)}{2}, \\
\Phi_{l-1 / 2, m}^{n+s}=\frac{f^{\prime}\left(U_{l, m}^{n+s}\right)+f^{\prime}\left(U_{l-1, m}^{n+s}\right)}{2}, \quad \Phi_{l, m-1 / 2}^{n+s}=\frac{f^{\prime}\left(U_{l, m}^{n}\right)+f^{\prime}\left(U_{l, m-1}^{n+s}\right)}{2} .
\end{gathered}
$$

The multilevel discretization consists of alternating $p$ steps on $4.2 \mathrm{a}$ with smaller time step $\Delta t / p$, from $t_{n}$ to $t_{n+1}$ followed by $q$ steps on $4.2 \mathrm{~b}$ with time step $\Delta t$, the incrementals being frozen at $t_{n+1}$ from $t_{n+1}$ to $t_{n+q+1}$. Then, using (4.1), we can go back to the fine mesh for $p$ steps from $t_{n+q+1}$ to $t_{n+q+2}$.

Since 4.2 is a succession of linear equation, the existence and uniqueness of solution follows the existence and uniqueness of solution discussed in section 3 Theorem 3.1 .

Theorem 4.1. The multilevel method defined by the equations $4.2 \mathrm{a}-4.2 \mathrm{e}$ is conditionally stable in $L^{\infty}\left(0, T ; \mathcal{H}_{h}\right)$, that is, if $\Delta t \leq \varepsilon^{2}$ and $1 \leq n \leq M$, then

$$
\left\|\boldsymbol{u}^{n}\right\|_{h}^{2} \leq 2 \frac{2 T}{\varepsilon^{2}}\left\|\boldsymbol{u}^{0}\right\|_{h}^{2}
$$

Proof. By multiplying 4.2a by $2 \frac{\Delta t}{p} \Delta x \Delta y u_{i, j}^{n+(r+1) / p}$ and adding the corresponding equalities for $i=$ $1, \ldots, 3 N_{1}^{0}$ and $j=1, \ldots, 3 N_{2}^{0}$, after the application of Lemmas 2.2 and 3.1 , we obtain

$$
\left\|\mathbf{u}^{n+(r+1) / p}\right\|_{h}^{2}-\left\|\mathbf{u}^{n+r / p}\right\|_{h}^{2}+\left\|\mathbf{u}^{n+(r+1) / p}-\mathbf{u}^{n+r / p}\right\|_{h}^{2}+2 \frac{\Delta t}{p} \varepsilon^{2}\left|\mathbf{u}^{n+(r+1) / p}\right|_{2, h}^{2} \leq \frac{2 \Delta t}{p}\left|\mathbf{u}^{n+(r+1) / p}\right|_{1, h}^{2} .
$$

And then using Young's inequality and Lemma 2.5. (4.3) yields

$$
\left\|\mathbf{u}^{n+(r+1) / p}\right\|_{h}^{2}-\left\|\mathbf{u}^{n+r / p}\right\|_{h}^{2}+\left\|\mathbf{u}^{n+(r+1) / p}-\mathbf{u}^{n+r / p}\right\|_{h}^{2} \leq \frac{\Delta t}{2 p \varepsilon^{2}}\left\|\mathbf{u}^{n+(r+1) / p}\right\|_{h}^{2} .
$$

Thus we have

$$
\left[1-\frac{\Delta t}{2 p \varepsilon^{2}}\right]\left\|\mathbf{u}^{n+(r+1) / p}\right\|_{h}^{2} \leq\left\|\mathbf{u}^{n+r / p}\right\|_{h}^{2}
$$

Based on 2.17 , for $\frac{\Delta t}{2 p \varepsilon^{2}} \leq \frac{1}{2}$, we have

$$
\left\|\mathbf{u}^{n+(r+1) / p}\right\|_{h}^{2} \leq 2^{\frac{2 \Delta t}{p \varepsilon^{2}}}\left\|\mathbf{u}^{n+r / p}\right\|_{h}^{2}
$$

After $p$ iterations, we obtain

$$
\left\|\mathbf{u}^{n+1}\right\|_{h}^{2} \leq 2^{\frac{2 \Delta t}{\varepsilon^{2}}}\left\|\mathbf{u}^{n}\right\|_{h}^{2} .
$$

We now perform $q$ iterations on the coarse grid, (4.2b), using time step $\Delta t$ and the relations (4.1). At time $t_{n+s}=(n+s) \Delta t, 2 \leq s \leq q+1$, the incremental unknowns $Z_{i, j}$ are frozen at time $(n+1) \Delta t$. Multiplying $4.2 \mathrm{~b}$ by $18 \Delta t \Delta x \Delta y U_{l, m}^{n+s+1}$ and adding the equalities for $l=1, \ldots, N_{1}^{0}$ and $m=1, \ldots, N_{2}^{0}$, together with Lemmas 2.5 and 3.1 and Young's inequality, we obtain

$$
\left\|\mathbf{U}^{n+s+1}\right\|_{3 h}^{2}-\left\|\mathbf{U}^{n+s}\right\|_{3 h}^{2}+\left\|\mathbf{U}^{n+s+1}-\mathbf{U}^{n+s}\right\|_{3 h}^{2} \leq \frac{\Delta t}{2 \varepsilon^{2}}\left\|\mathbf{U}^{n+s+1}\right\|_{3 h}^{2} .
$$


Thus we have

$$
\left[1-\frac{\Delta t}{2 \varepsilon^{2}}\right]\left\|\mathbf{U}^{n+s+1}\right\|_{3 h}^{2} \leq\left\|\mathbf{U}^{n+s}\right\|_{3 h}^{2}
$$

Using (2.17), for $\frac{\Delta t}{2 \varepsilon^{2}} \leq \frac{1}{2}$, we have

$$
\left\|\mathbf{U}^{n+s+1}\right\|_{3 h}^{2} \leq 2^{\frac{2 \Delta t}{\varepsilon^{2}}}\left\|\mathbf{U}^{n+s}\right\|_{3 h}^{2}
$$

From the definition of the increments $Z_{3 l-\alpha, 3 m-\beta}^{n+1}$, we have

$$
u_{3 l-\alpha, 3 m-\beta}^{n+s}=U_{l, m}^{n+s}+Z_{3 l-\alpha, 3 m-\beta}^{n+1}, \quad 1 \leq l \leq N_{1}^{0}, 1 \leq m \leq N_{2}^{0}, \quad \alpha, \beta=0,1,2 .
$$

Taking the sum over $\alpha$ and $\beta$, we get

$$
\sum_{\alpha, \beta=0}^{2}\left|u_{3 l-\alpha, 3 m-\beta}^{n+s}\right|^{2}=\sum_{\alpha, \beta=0}^{2}\left|U_{l, m}^{n+s}+Z_{3 l-\alpha, 3 m-\beta}^{n+1}\right|^{2}=9\left|U_{l, m}^{n+s}\right|^{2}+\sum_{\alpha, \beta=0}^{2}\left|Z_{3 l-\alpha, 3 m-\beta}^{n+1}\right|^{2} .
$$

For $s=1, \ldots, q+1$, the following relation holds

$$
\left\|\mathbf{u}^{n+s}\right\|_{h}^{2}=\left\|\mathbf{U}^{n+s}\right\|_{3 h}^{2}+\left\|\mathbf{Z}^{n+1}\right\|_{h}^{2}
$$

By adding $\left\|\mathbf{Z}^{n+1}\right\|_{h}^{2}$ to both sides of inequality 4.5) and using (4.6), we get

$$
\left\|\mathbf{u}^{n+s+1}\right\|_{h}^{2} \leq 2 \frac{2 \Delta t}{\varepsilon^{2}}\left\|\mathbf{u}^{n+s}\right\|_{h}^{2} .
$$

After $q$ iterations, and using (4.4), we have

$$
\left\|\mathbf{u}^{n+q+1}\right\|_{h}^{2} \leq 2 \frac{2 \Delta t(q+1)}{\varepsilon^{2}}\left\|\mathbf{u}^{n}\right\|_{h}^{2} .
$$

By induction over $n$, we obtain

$$
\left\|\mathbf{u}^{n}\right\|_{h}^{2} \leq 2^{\frac{2 n \Delta t}{\varepsilon^{2}}}\left\|\mathbf{u}^{0}\right\|_{h}^{2} \leq 2^{\frac{2 T}{\varepsilon^{2}}}\left\|\mathbf{u}^{0}\right\|_{h}^{2} .
$$

This completes the proof.

Theorem 4.2. Suppose that the solution $u(x, t)$ of $1.1-(1.4$ is sufficiently smooth. Assume that $\Delta t<4 \varepsilon^{2}, 4.9$, and 4.14 are satisfied.

Assume that $\Delta t, \Delta x$ and $\Delta y$ satisfy (4.11).

Then, the solution of the finite volume discretization 4.2a-4.2e converges to the solution of (1.1) in the discrete $L^{2}$-norm with rate of convergence $\mathcal{O}\left(\Delta t+(3 \Delta x)^{2}+(3 \Delta y)^{2}\right)$.

Proof. Let $n$ is a multiple of $q+1$. Let

$$
v_{i, j}^{n+r / p}=\iint_{k_{i, j}} u\left(x, y, t_{n+r / p}\right) d x d y
$$

be the cell average of $u$ at time $t_{n+r / p}$ on the cell $k_{i, j}$ for $1 \leq i \leq 3 N_{1}^{0}, 1 \leq j \leq 3 N_{2}^{0}, 0 \leq r \leq p$. Denote

$$
s=\max _{-L_{1} \leq x \leq L_{1},-L_{2} \leq y \leq L_{2}, 0 \leq t \leq T}|u(x, y, t)| .
$$


Making use of Taylor expansion, we obtain

$$
\begin{aligned}
\frac{v_{i, j}^{n+(r+1) / p}-v_{i, j}^{n+r / p}}{\Delta t / p}-\left(C_{h}\left(\boldsymbol{v}^{n+(r+1) / p}, \tilde{\boldsymbol{v}}^{n+r / p}\right)\right)_{i, j} & +\varepsilon^{2} \Delta_{h}^{2} v_{i, j}^{n+(r+1) / p}=\nabla_{1, h}^{+}\left[\psi_{i-\frac{1}{2}, j}^{n+r / p} \nabla_{1, h}^{-} v_{i, j}^{n+(r+1) / p}\right] \\
& +\nabla_{2, h}^{+}\left[\psi_{i, j-\frac{1}{2}}^{n+r / p} \nabla_{2, h}^{-} v_{i, j}^{n+(r+1) / p}\right]+\tau_{i, j}^{n+r / p},
\end{aligned}
$$

where $\tau_{i, j}^{n+r / p} \in \mathcal{H}_{h}$ is the truncation error of the finite volume discretization $4.2 \mathrm{a}$ for $1 \leq i \leq 3 N_{1}^{0}, 1 \leq j \leq$ $3 N_{2}^{0}$. There exists a positive constant $c_{1}$ such that

$$
\max _{i, j, n}\left|\tau_{i, j}^{n+r / p}\right| \leq c_{1}\left(\frac{\Delta t}{p}+\Delta x^{2}+\Delta y^{2}\right), 1 \leq r \leq p \text { and } \leq 1 \leq i \leq 3 N_{0} .
$$

Let $\mathbf{e}^{n+r / p}=\boldsymbol{v}^{n+r / p}-\mathbf{u}^{n+r / p}$, where $u_{i, j}^{n+r / p}$ is the solution of $4.2 \mathrm{a}$. Substituting $u_{i, j}^{n+r / p}=v_{i, j}^{n+r / p}-e_{i, j}^{n+r / p}$ in 4.2a), and using (4.7), we obtain

$$
\begin{aligned}
\frac{e_{i, j}^{n+(r+1) / p}-e_{i, j}^{n+r / p}}{\Delta t / p} & -\left(C_{h}\left(\mathbf{e}^{n+(r+1) / p}, \tilde{\boldsymbol{v}}^{n+r / p}-\tilde{\mathbf{e}}^{n+r / p}\right)\right)_{i, j}+\varepsilon^{2} \Delta_{h}^{2} e_{i, j}^{n+(r+1) / p}=\left(C_{h}\left(\boldsymbol{v}^{n+(r+1) / p}, \tilde{\mathbf{e}}^{n+r / p}\right)\right)_{i, j} \\
& +\nabla_{1, h}^{+}\left[\left(3\left(v_{i, j}^{n+r / p} e_{i, j}^{n+r / p}+v_{i-1, j}^{n+r / p} e_{i-1, j}^{n+r / p}\right)-\frac{3}{2}\left[\left(e_{i, j}^{n+r / p}\right)^{2}+\left(e_{i-1, j}^{n+r / p}\right)^{2}\right]\right) \nabla_{1, h}^{-} v_{i, j}^{n+(r+1) / p}\right] \\
& +\nabla_{2, h}^{+}\left[\left(3\left(v_{i, j}^{n+r / p} e_{i, j}^{n+r / p}+v_{i, j-1}^{n+r / p} e_{i, j-1}^{n+r / p}\right)-\frac{3}{2}\left[\left(e_{i, j}^{n+r / p}\right)^{2}+\left(e_{i, j-1}^{n+r / p}\right)^{2}\right]\right) \nabla_{2, h}^{-} v_{i, j}^{n+(r+1) / p}\right] \\
& +\nabla_{1, h}^{+}\left(\varphi_{i-\frac{1}{2}, j}^{n+r / p} \nabla_{1, h}^{-} e_{i, j}^{n+(r+1) / p}\right)+\nabla_{2, h}^{+}\left(\varphi_{i, j-\frac{1}{2}}^{n+r / p} \nabla_{2, h}^{-} e_{i, j}^{n+(r+1) / p}\right)+\tau_{i, j}^{n+r / p} .
\end{aligned}
$$

Multiplying 4.8 by $\frac{2 \Delta t \Delta x \Delta y}{p} e_{i, j}^{n+(r+1) / p}$ and summing for $i=1, \ldots, 3 N_{1}^{0}$ and $j=1, \ldots, 3 N_{2}^{0}$, together with 2.15 and Lemmas 3.1 and 2.1, we obtain

$$
\begin{aligned}
& \left\|\mathbf{e}^{n+(r+1) / p}\right\|_{h}^{2}-\left\|\mathbf{e}^{n+r / p}\right\|_{h}^{2}+\left\|\mathbf{e}^{n+(r+1) / p}-\mathbf{e}^{n+r / p}\right\|_{h}^{2}+\frac{2 \Delta t}{p} \varepsilon^{2}\left\|\mathbf{e}^{n+(r+1) / p}\right\|_{2, h}^{2} \leq \frac{2 \Delta t}{p}\left\|\mathbf{e}_{i, j}^{n+(r+1) / p}\right\|_{1, h}^{2} \\
& -\frac{2 \Delta t \Delta x \Delta y}{p} \sum_{i=1}^{3 N_{1}^{0}} \sum_{j=1}^{3 N_{2}^{0}}\left[\left(3\left(v_{i, j}^{n+r / p} e_{i, j}^{n+r / p}+v_{i-1, j}^{n+r / p} e_{i-1, j}^{n+r / p}\right)-\frac{3}{2}\left[\left(e_{i, j}^{n+r / p}\right)^{2}+\left(e_{i-1, j}^{n+r / p}\right)^{2}\right]\right) \nabla_{1, h}^{-} v_{i, j}^{n+(r+1) / p}\right] \nabla_{1, h}^{-} e_{i, j}^{n+(r+1) / p} \\
& -\frac{2 \Delta t \Delta x \Delta y}{p} \sum_{i=1}^{3 N_{1}^{0}} \sum_{j=1}^{3 N_{2}^{0}}\left[\left(3\left(v_{i, j}^{n+r / p} e_{i, j}^{n+r / p}+v_{i, j-1}^{n+r / p} e_{i, j-1}^{n+r / p}\right)-\frac{3}{2}\left[\left(e_{i, j}^{n+r / p}\right)^{2}+\left(e_{i, j-1}^{n+r / p}\right)^{2}\right]\right) \nabla_{2, h}^{-} v_{i, j}^{n+(r+1) / p}\right] \nabla_{2, h}^{-} e_{i, j}^{n+(r+1) / p} \\
& +\frac{2 \Delta t}{p}\left(C_{h}\left(\boldsymbol{v}^{n+(r+1) / p}, \tilde{\mathbf{e}}^{n+r / p}\right), \mathbf{e}^{n+(r+1) / p}\right)_{h}+\frac{2 \Delta t}{p}\left(\boldsymbol{\tau}^{n+r / p}, \mathbf{e}^{n+(r+1) / p}\right)_{h} .
\end{aligned}
$$

Using the approach implemented on the proof of Theorem 3.3 we deduce in the fine mesh taking

$$
\Delta t c \leq \frac{p}{2},
$$

then

$$
\begin{aligned}
\left\|\mathbf{e}^{n+(r+1) / p}\right\|_{h}^{2} & +\frac{1}{p} \Delta t \varepsilon^{2} \eta^{2}\left|\mathbf{e}^{n+(r+1) / p}\right|_{1, h}^{2} \leq \frac{3 \Delta t \delta_{2} 4^{\Delta t c}}{p \Delta x \Delta y}\left\|\mathbf{e}^{n+r / p}\right\|_{h}^{2}\left|\mathbf{e}^{n+(r+1) / p}\right|_{1, h}^{2} \\
& +4^{\Delta t c}\left[\left(1+\frac{\Delta t c_{9}}{p}\right)\left\|\mathbf{e}^{n+r / p}\right\|_{h}^{2}+\frac{\Delta t c_{8}}{p}\left\|\tilde{\mathbf{e}}^{n+r / p}\right\|_{h}^{2}+\frac{\Delta t}{p} c_{7}\left(\frac{\Delta t}{p}+\Delta x^{2}+\Delta y^{2}\right)^{2}\right] .
\end{aligned}
$$

For

$$
\frac{3 \delta_{2} c_{7}}{\Delta x \Delta y}\left(\frac{\Delta t}{p}+\Delta x^{2}+\Delta y^{2}\right)^{2} \leq \frac{1}{2 T} \varepsilon^{2} \eta^{2} 4^{-T c} \exp \left(-T c_{10}\right)
$$


as shown in Theorem 3.3 , we obtain

$$
\begin{aligned}
\left\|\mathbf{e}^{n+(r+1) / p}\right\|_{h}^{2} & +\frac{1}{2 p} \Delta t \varepsilon^{2} \eta^{2}\left|\mathbf{e}^{n+(r+1) / p}\right|_{1, h}^{2} \\
& \leq 4 \frac{\Delta t c}{p}\left[\left(1+\frac{\Delta t c_{9}}{p}\right)\left\|\mathbf{e}^{n+r / p}\right\|_{h}^{2}+\frac{\Delta t c_{8}}{p}\left\|\tilde{\mathbf{e}}^{n+r / p}\right\|_{h}^{2}+\frac{\Delta t}{p} c_{7}\left(\frac{\Delta t}{p}+\Delta x^{2}+\Delta y^{2}\right)^{2}\right]
\end{aligned}
$$

which after $p$ iterations gives

$$
\left\|\mathbf{e}^{n+1}\right\|_{h}^{2} \leq 4^{\Delta t c} \exp \left(\Delta t c_{10}\right)\left[\left\|\mathbf{e}^{n}\right\|_{h}^{2}+\Delta t c_{7}\left(\frac{\Delta t}{p}+\Delta x^{2}+\Delta y^{2}\right)^{2}\right] .
$$

In a similar way for $1 \leq s \leq q$, and being on the coarse mesh and for

$$
\Delta t c \leq \frac{1}{2}
$$

we get

$$
\left\|\mathbf{E}^{n+s+1}\right\|_{3 h}^{2}+\frac{1}{2} \Delta t \varepsilon^{2} \eta^{2}\left|\mathbf{E}^{n+s+1}\right|_{1,3 h}^{2} \leq 4^{\Delta t c}\left[\left(1+\Delta t c_{9}\right)\left\|\mathbf{E}^{n+s}\right\|_{3 h}^{2}+\Delta t c_{8}\left\|\tilde{\mathbf{E}}^{n+s}\right\|_{3 h}^{2}+\Delta t c_{7}\left(\Delta t+(3 \Delta x)^{2}+(3 \Delta y)^{2}\right)^{2}\right],
$$

where $\mathbf{E}^{n+s}=\boldsymbol{\Upsilon}^{n+s}-\mathbf{U}^{n+s}$ and $\boldsymbol{\Upsilon}^{n+s}$ and $\mathbf{U}^{n+s}$ are exact cell average and numerical solutions on the coarse mesh, respectively. For $n+s<m_{0}-1$, we have

$$
\left\|\mathbf{E}^{n+s+1}\right\|_{3 h}^{2} \leq 4^{\Delta t c} \exp \left(\Delta t\left(c_{9}+c_{8}\right)\right)\left[\left\|\mathbf{E}^{n+s}\right\|_{3 h}^{2}+\Delta t c_{7}\left(\Delta t+(3 \Delta x)^{2}+(3 \Delta y)^{2}\right)^{2}\right],
$$

As we said at the beginning of this section, the numerical increments $Z_{i, j}$ 's are fixed between steps $n+1$ and $n+q+1$ and therefore for $1 \leq s \leq q, 1 \leq l \leq N_{1}^{0}$ and $1 \leq m \leq N_{2}^{0}$,

$$
Z_{3 l-\alpha, 3 m-\beta}^{n+s+1}=Z_{3 l-\alpha, 3 m-\beta}^{n+1}=u_{3 l-\alpha, 3 m-\beta}^{n+1}-U_{l, m}^{n+1}, \quad \alpha, \beta=0,1,2 .
$$

Using 4.1, we have

$$
e_{3 l-\alpha, 3 m-\beta}^{n+s+1}=E_{l, m}^{n+s+1}+\zeta_{3 l-\alpha, 3 m-\beta}^{n+s+1},
$$

where

$$
\zeta_{3 l-\alpha, 3 m-\beta}^{n+s+1}=\left(v_{3 l-\alpha, 3 m-\beta}^{n+s+1}-\Upsilon_{l, m}^{n+s+1}\right)-Z_{3 l-\alpha, 3 m-\beta}^{n+1},
$$

is the difference of the numerical increment from exact increment. It is clear from the definition of increments that

$$
\sum_{\alpha, \beta=0}^{2}\left(v_{3 l-\alpha, 3 m-\beta}^{n+s+1}-\Upsilon_{l, m}^{n+s+1}\right)=\sum_{\alpha, \beta=0}^{2} Z_{3 l-\alpha, 3 m-\beta}^{n+1}=0
$$

and hence $\sum_{\alpha, \beta=0}^{2} \zeta_{3 l-\alpha, 3 m-\beta}^{n+s+1}=0$. As a result for $s=1, \ldots, q$,

$$
\sum_{\alpha, \beta=0}^{2}\left(e_{3 l-\alpha, 3 m-\beta}^{n+s+1}\right)^{2}=9\left(E_{l, m}^{n+s+1}\right)^{2}+\sum_{\alpha, \beta=0}^{2}\left(\zeta_{3 l-\alpha, 3 m-\beta}^{n+s+1}\right)^{2} .
$$


Multiplying 4.17) by $\Delta x \Delta y$ and taking the sum for $l=1, \ldots, N_{1}^{0}$ and $m=1, \ldots, N_{2}^{0}$, we obtain

$$
\left\|\mathbf{e}^{n+s+1}\right\|_{h}^{2}=\left\|\mathbf{E}^{n+s+1}\right\|_{3 h}^{2}+\left\|\boldsymbol{\zeta}^{n+s+1}\right\|_{h}^{2} .
$$

We now estimate the term $\boldsymbol{\zeta}^{n+s+1}$. From the definition of increments for $\mathbf{u} \in \mathcal{H}_{h}$, we have

$$
U_{l, m}=u_{3 l-\alpha, 3 m-\beta}+\mathcal{O}(\Delta x+\Delta y)
$$

from which

$$
\Upsilon_{l, m}^{n+s+1}-v_{3 l-\alpha, 3 m-\beta}^{n+s+1}=\Upsilon_{l, m}^{n+s}-v_{3 l-\alpha, 3 m-\beta}^{n+s}+\Delta t \mathcal{O}(\Delta x+\Delta y)
$$

Hence

$$
\zeta^{n+s+1}=\zeta^{n+s}+\Delta t \mathcal{O}(\Delta x+\Delta y)
$$

(4.20) gives

$$
\left\|\boldsymbol{\zeta}^{n+s+1}\right\|_{h}^{2}=\left\|\boldsymbol{\zeta}^{n+s}\right\|_{h}^{2}+\Delta t \mathcal{O}\left(\Delta t(\Delta x+\Delta y)^{2}\right)
$$

which with the application of Young's inequality, 2.18 gives

$$
\left\|\boldsymbol{\zeta}^{n+s+1}\right\|_{h}^{2} \leq\left\|\boldsymbol{\zeta}^{n+s}\right\|_{h}^{2}+\Delta t c_{11}\left(\Delta t+\Delta x^{2}+\Delta y^{2}\right)^{2},
$$

where $c_{11}$ is a constant independent of $\Delta t, \Delta x$ and $\Delta y$.

Combining 4.16), 4.18 and 4.21, we obtain

$$
\left\|\mathbf{e}^{n+s+1}\right\|_{h}^{2} \leq 4^{\Delta t c} \exp \left(\Delta t\left(c_{9}+c_{8}\right)\right)\left[\left\|\mathbf{e}^{n+s}\right\|_{h}^{2}+\Delta t c_{12}\left(\Delta t+(3 \Delta x)^{2}+(3 \Delta y)^{2}\right)^{2}\right],
$$

which after $s$ iterations gives

$$
\left\|\mathbf{e}^{n+s+1}\right\|_{h}^{2} \leq 4^{\Delta t s c} \exp \left(\Delta t s\left(c_{9}+c_{8}\right)\right)\left[\left\|\mathbf{e}^{n+1}\right\|_{h}^{2}+\Delta t s c_{12}\left(\Delta t+(3 \Delta x)^{2}+(3 \Delta y)^{2}\right)^{2}\right],
$$

where $c_{12}=\max \left\{c_{7}, 4^{-\Delta t c} \exp \left(-\Delta t\left(c_{9}+c_{8}\right)\right) c_{11}\right\}$. Together with 4.13, 4.22 becomes

$$
\begin{gathered}
\left\|\mathbf{e}^{n+s+1}\right\|_{h}^{2} \leq 4^{\Delta t(s+1) c} \exp \left[\Delta t s\left(c_{9}+c_{8}\right)\right]\left[\exp \left(\Delta t c_{10}\right)\left[\left\|\mathbf{e}^{n}\right\|_{h}^{2}+\Delta t c_{12}\left(\Delta t+\Delta x^{2}+\Delta y^{2}\right)^{2}\right]\right. \\
\left.+\Delta t s c_{12}\left(\Delta t+(3 \Delta x)^{2}+(3 \Delta y)^{2}\right)^{2}\right] .
\end{gathered}
$$

Since $m_{0} A \geq 1$, it follows from this inequality that

$$
\left\|\mathbf{e}^{n+s+1}\right\|_{h}^{2} \leq 4^{\Delta t(s+1) c} \exp \left(\Delta t(s+1) c_{10}\right)\left[\left\|\mathbf{e}^{n}\right\|_{h}^{2}+\Delta t(s+1) c_{12}\left(\Delta t+(3 \Delta x)^{2}+(3 \Delta y)^{2}\right)^{2}\right] .
$$

Thus, after $n$ iterations, we get

$$
\left\|\mathbf{e}^{n+s+1}\right\|_{h}^{2} \leq 4^{\Delta t(n+s+1) c} \exp \left(\Delta t(n+s+1)\left(c_{10}\right)\right)\left[\Delta t(n+s+1) c_{12}\left(\Delta t+(3 \Delta x)^{2}+(3 \Delta y)^{2}\right)^{2}\right] .
$$

For the case $n+s \geq m_{0}-1$, we have

$$
\begin{gathered}
\left\|\mathbf{E}^{n+s+1}\right\|_{3 h}^{2} \leq 4^{\Delta t c}\left(1+\Delta t c_{9}\right)\left\|\mathbf{E}^{n+s}\right\|_{3 h}^{2}+\Delta t A c_{8} 4^{\Delta t c}\left[\left\|\mathbf{E}^{n+s}\right\|_{3 h}^{2}+\left\|\mathbf{E}^{n+s-1}\right\|_{3 h}^{2}+\cdots+\left\|\mathbf{E}^{n+s-m_{0}+1}\right\|_{3 h}^{2}\right] \\
+\Delta t c_{12} 4^{\Delta t c}\left(\Delta t+(3 \Delta x)^{2}+(3 \Delta y)^{2}\right)^{2}
\end{gathered}
$$


which implies

$$
\begin{gathered}
\max \left\{\left\|\mathbf{E}^{n+s+1}\right\|_{3 h}^{2}, \ldots,\left\|\mathbf{E}^{n+s-m_{0}+2}\right\|_{3 h}^{2},\right\} \leq 4^{\Delta t c} \exp \left(\Delta t c_{10}\right) \max \left\{\left\|\mathbf{E}^{n+s}\right\|_{3 h}^{2},\left\|\mathbf{E}^{n+s-1}\right\|_{3 h}^{2}, \ldots,\left\|\mathbf{E}^{n+s-m_{0}+1}\right\|_{3 h}^{2}\right\} \\
+\Delta t c_{12} 4^{\Delta t c}\left(\Delta t+(3 \Delta x)^{2}+(3 \Delta y)^{2}\right)^{2}
\end{gathered}
$$

Using (4.18, 4.21) and 4.25), we obtain

$$
\begin{aligned}
\max \{ & \left.\left\|\mathbf{e}^{n+s+1}\right\|_{h}^{2}, \ldots,\left\|\mathbf{e}^{n+s-m_{0}+2}\right\|_{h}^{2}\right\} \\
\leq & 4^{\Delta t c} \exp \left(c_{10}\right) \max \left\{\left\|\mathbf{e}^{n+s}\right\|_{h}^{2},\left\|\mathbf{e}^{n+s-1}\right\|_{h}^{2}, \ldots,\left\|\mathbf{e}^{n+s-m_{0}+1}\right\|_{h}^{2}\right\}+\Delta t c_{12} 4^{\Delta t c}\left(\Delta t+(3 \Delta x)^{2}+(3 \Delta y)^{2}\right)^{2} \\
\leq & 4^{\Delta t\left(n+s-m_{0}+1\right) c} \exp \left[\Delta t c_{10}\left(n+s-m_{0}+1\right)\right]\left[\max \left\{\left\|\mathbf{e}^{m_{0}-1}\right\|_{h}^{2},\left\|\mathbf{e}^{m_{0}-2}\right\|_{h}^{2}, \ldots,\left\|\mathbf{e}^{0}\right\|_{h}^{2}\right\}\right. \\
& \left.\quad+\Delta t\left(n+s-m_{0}+1\right) c_{12}\left(\Delta t+(3 \Delta x)^{2}+(3 \Delta y)^{2}\right)^{2}\right]
\end{aligned}
$$

Using (4.23), 4.26) and induction on $n$, one obtains

$$
\begin{aligned}
\left\|\mathbf{e}^{n}\right\|_{h}^{2} & \leq 4^{\Delta t(n) c} \exp \left[n \Delta t\left(c_{10}\right)\right]\left[\left\|\mathbf{e}^{0}\right\|_{h}^{2}+n \Delta t c_{12}\left(\Delta t+(3 \Delta x)^{2}+(3 \Delta y)^{2}\right)^{2}\right] \\
& \leq 4^{T c} \exp \left[T\left(c_{10}\right)\right]\left[T c_{12}\left(\Delta t+(3 \Delta x)^{2}+(3 \Delta y)^{2}\right)^{2}\right],
\end{aligned}
$$

for $n=1, \ldots, M$. Therefore, we have

$$
\left\|\mathbf{e}^{n}\right\|_{h} \leq C\left(\Delta t+(3 \Delta x)^{2}+(3 \Delta y)^{2}\right)
$$

where $C$ is a constant independent of $\Delta t, \Delta x$ and $\Delta y$. This completes the proof.

The rate of convergence depends on the mesh size of the coarse mesh.

\subsection{Explicit Multilevel Finite Volume Method}

For $0 \leq r \leq p-1$ and $1 \leq s \leq q$, we discretize (1.1) using explicit multilevel finite volume method.

$$
\begin{aligned}
& \frac{p}{\Delta t}\left(u_{i, j}^{n+(r+1) / p}-u_{i, j}^{n+r / p}\right)-\left(C_{h}\left(\mathbf{u}^{n+r / p}, \mathbf{u}^{n+r / p}\right)\right)_{i, j}+\varepsilon^{2} \Delta_{h}^{2} u_{i, j}^{n+r / p} \\
& =\nabla_{1, h}^{+}\left(\varphi_{i-1 / 2, j}^{n+r / p} \nabla_{1, h}^{-} u_{i, j}^{n+r+/ p}\right)+\nabla_{2, h}^{+}\left(\varphi_{i, j-1 / 2}^{n+r / p} \nabla_{2, h}^{-} u_{i, j}^{n+r / p}\right) \text {, } \\
& \frac{U_{l, m}^{n+s+1}-U_{l, m}^{n+s}}{\Delta t}-\left(C_{3 h}\left(\mathbf{U}^{n+s}, \mathbf{U}^{n+s}\right)\right)_{l, m}+\varepsilon^{2} \Delta_{3 h}^{2} U_{l, m}^{n+s} \\
& =\nabla_{1,3 h}^{+}\left(\Phi_{l-1 / 2, m}^{n+s} \nabla_{1,3 h}^{-} U_{l, m}^{n+s}\right)+\nabla_{2,3 h}^{+}\left(\Phi_{l, m-1 / 2}^{n+s} \nabla_{2,3 h}^{-} U_{l, m}^{n+s}\right) . \\
& u_{i, j}^{n+r / p}=u_{i+3 N_{1}^{0}, j}^{n+r / p}=u_{i, j+3 N_{2}^{0}}^{n+r / p} \text {, } \\
& U_{l, m}^{n+s}=U_{l+N_{1}^{0}, m}^{n+s}=U_{l, m+N_{2}^{0}}^{n+s}, \\
& u_{i, j}^{0}=\frac{1}{\Delta x \Delta y} \iint_{k_{i, j}} u^{0}(x, y) d x d y \text {, }
\end{aligned}
$$

where $1 \leq i \leq 3 N_{1}^{0}, 1 \leq l \leq N_{1}^{0}, 1 \leq j \leq 3 N_{2}^{0}$ and $1 \leq m \leq N_{2}^{0}$. 
Theorem 4.3. We assume that the following satisfied for some $\delta, 0<\delta<1$ :

$$
\begin{aligned}
& 32 \Delta t\left(\frac{1}{\Delta x^{2}}+\frac{1}{\Delta y^{2}}\right)^{2} \leq \frac{1-\delta}{2 \varepsilon^{2}} \min \{p, 81\}, \\
& 16 \Delta t\left(\frac{1}{\Delta x^{2}}+\frac{1}{\Delta y^{2}}\right) \leq \varepsilon^{2} \delta \eta^{2}(1-\delta) \min \{p, 9\}, \\
& \frac{72 \Delta t}{p \Delta x \Delta y}\left(\left(\left|\alpha_{1}\right|+\left|\alpha_{2}\right|\right)^{2}+\frac{4}{\Delta x \Delta y}\left(\frac{1}{\Delta x^{2}}+\frac{1}{\Delta y^{2}}\right)\left\|\boldsymbol{u}^{0}\right\|_{h}^{2}\right)\left\|\boldsymbol{u}^{0}\right\|_{h}^{2} \leq \varepsilon^{2} \delta^{2} \eta^{2} \exp \left(\frac{-2 T}{\varepsilon^{2}}\right), \\
& \frac{8 \Delta t}{\Delta x \Delta y}\left(\left(\left|\alpha_{1}\right|+\left|\alpha_{2}\right|\right)^{2}+\frac{4}{81 \Delta x \Delta y}\left(\frac{1}{\Delta x^{2}}+\frac{1}{\Delta y^{2}}\right)\left\|\boldsymbol{u}^{0}\right\|_{h}^{2}\right)\left\|\boldsymbol{u}^{0}\right\|_{h}^{2} \leq \varepsilon^{2} \delta^{2} \eta^{2} \exp \left(\frac{-2 T}{\varepsilon^{2}}\right) .
\end{aligned}
$$

Then the multilevel method defined by the equations 4.28a - 4.28e is $L^{\infty}\left(0, T ; \mathcal{H}_{h}\right)$ stale in the following sense:

$$
\begin{aligned}
& \left\|\boldsymbol{u}^{n}\right\|^{2} \leq \exp \left(\frac{\Delta t}{\varepsilon^{2}}\right)\left\|\boldsymbol{u}^{n-1}\right\|^{2} \leq \cdots \leq \exp \left(\frac{n \Delta t}{\varepsilon^{2}}\right)\left\|\boldsymbol{u}^{0}\right\|^{2} \leq \exp \left(\frac{T}{\varepsilon^{2}}\right)\left\|\boldsymbol{u}^{0}\right\|^{2}, \quad n=1,2 \ldots, M_{0}, \\
& \left\|\boldsymbol{u}^{s(q+1)+r / p}\right\|^{2} \leq \exp \left(\frac{r \Delta t}{p \varepsilon^{2}}\right)\left\|\boldsymbol{u}^{s(q+1)}\right\|^{2}, \quad r=1,2, \ldots, p
\end{aligned}
$$

Proof. To prove this theorem we use the approach discussed in Theorem 3.4. We assume $n$ is a multiple of $q+1$. Multiplying 4.28a by $2 \frac{\Delta t}{p} \Delta x \Delta y u_{i, j}^{n+r / p}$ and taking the sum for $i=1, \ldots, 3 N_{1}^{0}$ and $j=1, \ldots, 3 N_{2}^{0}$ together with 2.16) and Lemma 2.5, we obtain

$$
\left\|\mathbf{u}^{n+(r+1) / p}\right\|_{h}^{2}-\left\|\mathbf{u}^{n+(r+1) / p}-\mathbf{u}^{n+r / p}\right\|_{h}^{2}+\frac{\Delta t}{p} \varepsilon^{2}\left|\mathbf{u}^{n+r / p}\right|_{2, h}^{2} \leq \exp \left(\frac{\Delta t}{p \varepsilon^{2}}\right)\left\|\mathbf{u}^{n+r / p}\right\|_{h}^{2} .
$$

To estimate the term $\left\|\mathbf{u}^{n+(r+1) / p}-\mathbf{u}^{n+r / p}\right\|_{h}^{2}$, we multiply 4.28a by $2 \frac{\Delta t}{p} \Delta x \Delta y\left(u_{i, j}^{n+(r+1) / p}-u_{i, j}^{n+r / p}\right)$ and summing from $i=1$ to $i=3 N_{1}^{0}$ and from $j=1$ to $j=3 N_{2}^{0}$, we find

$$
\begin{aligned}
\left\|\mathbf{u}^{n+(r+1) / p}-\mathbf{u}^{n+r / p}\right\|_{h}^{2} \leq & \frac{36 \Delta t^{2}}{p^{2} \Delta x \Delta y}\left(\left|\alpha_{1}\right|+\left|\alpha_{2}\right|\right)^{2}\left\|\mathbf{u}^{n+r / p}\right\|_{h}^{2}\left|\mathbf{u}^{n+r / p}\right|_{1, h}^{2}+\frac{64 \Delta t^{2}}{p^{2}}\left(\frac{1}{\Delta x^{2}}+\frac{1}{\Delta y^{2}}\right)^{2} \varepsilon^{4}\left|\mathbf{u}^{n+r / p}\right|_{2, h}^{2} \\
& \quad \frac{144 \Delta t^{2}}{p^{2} \Delta x^{2} \Delta y^{2}}\left(\frac{1}{\Delta x^{2}}+\frac{1}{\Delta y^{2}}\right)\left\|\mathbf{u}^{n+r / p}\right\|_{h}^{4}\left|\mathbf{u}^{n}\right|_{1, h}^{2}+\frac{16 \Delta t^{2}}{p^{2}}\left(\frac{1}{\Delta x^{2}}+\frac{1}{\Delta y^{2}}\right)\left|\mathbf{u}^{n+r / p}\right|_{1, h}^{2}
\end{aligned}
$$

Using 4.29, we obtain

$$
\begin{aligned}
\left\|\mathbf{u}^{n+(r+1) / p}-\mathbf{u}^{n+r / p}\right\|_{h}^{2} & \leq \frac{36 \Delta t^{2}}{p^{2} \Delta x \Delta y}\left(\left|\alpha_{1}\right|+\left|\alpha_{2}\right|\right)^{2}\left\|\mathbf{u}^{n}\right\|_{h}^{2}\left|\mathbf{u}^{n+r / p}\right|_{1, h}^{2}+\frac{\Delta t}{p} \varepsilon^{2}(1-\delta)\left|\mathbf{u}^{n+r / p}\right|_{2, h}^{2} \\
& +144 \frac{\Delta t^{2}}{p^{2} \Delta x^{2} \Delta y^{2}}\left(\frac{1}{\Delta x^{2}}+\frac{1}{\Delta y^{2}}\right)\left\|\mathbf{u}^{n+r / p}\right\|_{h}^{4}\left|\mathbf{u}^{n+r / p}\right|_{1, h}^{2}+\frac{16 \Delta t^{2}}{p^{2} h^{2}}\left|\mathbf{u}^{n+r / p}\right|_{1, h}^{2} .
\end{aligned}
$$

On substitution of 4.36 into 4.35, we get

$$
\begin{aligned}
\left\|\mathbf{u}^{n+(r+1) / p}\right\|_{h}^{2}+ & \frac{\Delta t}{p} \varepsilon^{2} \delta\left|\mathbf{u}^{n+r / p}\right|_{2, h}^{2} \leq \exp \left(\frac{\Delta t}{p \varepsilon^{2}}\right)\left\|\mathbf{u}^{n+r / p}\right\|_{h}^{2} \\
& +\frac{36 \Delta t^{2}}{p^{2} \Delta x \Delta y}\left[\left(\left|\alpha_{1}\right|+\left|\alpha_{2}\right|\right)^{2}+\frac{4}{\Delta x \Delta y}\left(\frac{1}{\Delta x^{2}}+\frac{1}{\Delta y^{2}}\right)\left\|\mathbf{u}^{n+r / p}\right\|_{h}^{2}\right]\left\|\mathbf{u}^{n+r / p}\right\|_{h}^{2}\left|\mathbf{u}^{n+r / p}\right|_{1, h}^{2}
\end{aligned}
$$


Using 2.24 and 4.30 , we obtain

$$
\begin{aligned}
\left\|\mathbf{u}^{n+(r+1) / p}\right\|_{h}^{2} & -\exp \left(\frac{\Delta t}{p \varepsilon^{2}}\right)\left\|\mathbf{u}^{n+r / p}\right\|_{h}^{2}+\frac{\Delta t}{p} \varepsilon^{2} \delta^{2} \eta^{2}\left|\mathbf{u}^{n+r / p}\right|_{1, h}^{2} \\
& -\frac{36 \Delta t^{2}}{p^{2} \Delta x \Delta y}\left[\left(\left|\alpha_{1}\right|+\left|\alpha_{2}\right|\right)^{2}+\frac{4}{\Delta x \Delta y}\left(\frac{1}{\Delta x^{2}}+\frac{1}{\Delta y^{2}}\right)\left\|\mathbf{u}^{n+r / p}\right\|_{h}^{2}\right]\left\|\mathbf{u}^{n+r / p}\right\|_{h}^{2}\left|\mathbf{u}^{n+r / p}\right|_{1, h}^{2} \leq 0 .
\end{aligned}
$$

In a similar fashion, from 4.28b together with the assumptions 4.29, 4.30 and 4.32, we obtain

$$
\begin{aligned}
\left\|\mathbf{U}^{n+m+1}\right\|_{3 h}^{2} & -\exp \left(\frac{\Delta t}{\varepsilon^{2}}\right)\left\|\mathbf{U}^{n+m}\right\|_{3 h}^{2}+\Delta t \varepsilon^{2} \delta^{2} \eta^{2}\left|\mathbf{U}^{n+m}\right|_{1,3 h}^{2} \\
& \leq \frac{4 \Delta t^{2}}{\Delta x \Delta y}\left[\left(\left|\alpha_{1}\right|+\left|\alpha_{2}\right|\right)^{2}+\frac{4}{81 \Delta x \Delta y}\left(\frac{1}{\Delta x^{2}}+\frac{1}{\Delta y^{2}}\right)\left\|\mathbf{U}^{n+m}\right\|_{3 h}^{2}\right]\left\|\mathbf{U}^{n+m}\right\|_{3 h}^{2}\left|\mathbf{U}^{n+m}\right|_{1,3 h}^{2} .
\end{aligned}
$$

Now we need to prove the following by induction on $n$

$$
\begin{array}{r}
\left\|\mathbf{u}^{n+(r+1) / p}\right\|_{h}^{2}+\frac{\Delta t}{2 p} \varepsilon^{2} \delta^{2} \eta^{2}\left|\mathbf{u}^{n+r / p}\right|_{1, h}^{2} \leq \exp \left(\frac{\Delta t}{p \varepsilon^{2}}\right)\left\|\mathbf{u}^{n+r / p}\right\|_{h}^{2}, \quad \text { for } r=0,1, \ldots, p-1, \\
\left\|\mathbf{U}^{n+s+1}\right\|_{3 h}^{2}+\frac{\Delta t}{2} \varepsilon^{2} \delta^{2} \eta^{2}\left|\mathbf{U}^{n+s}\right|_{1,3 h}^{2} \leq \exp \left(\frac{\Delta t}{\varepsilon^{2}}\right)\left\|\mathbf{U}^{n+s}\right\|_{3 h}^{2}, \quad \text { for } s=1,2, \ldots, q .
\end{array}
$$

We first show 4.39 and 4.40 hold by induction on $r$ and $s$ when $n=0$. We first show

$$
\left\|\mathbf{u}^{1}\right\|_{h}^{2}+\frac{\Delta t}{2 p} \varepsilon^{2} \delta^{2} \eta^{2} \sum_{r=0}^{p-1} \exp \left(\frac{(p-1-r) \Delta t}{p \varepsilon^{2}}\right)\left|\mathbf{u}^{r / p}\right|_{1, h}^{2} \leq \exp \left(\frac{\Delta t}{p \varepsilon^{2}}\right)\left\|\mathbf{u}^{r / p}\right\|_{h}^{2} .
$$

For $n=0$, the relation 4.37 becomes

$$
\begin{aligned}
\left\|\mathbf{u}^{(r+1) / p}\right\|_{h}^{2}+\frac{\Delta t}{p} \varepsilon^{2} \delta^{2} \eta^{2}\left|\mathbf{u}^{r / p}\right|_{1, h}^{2} \leq \exp \left(\frac{\Delta t}{p \varepsilon^{2}}\right)\left\|\mathbf{u}^{r / p}\right\|_{h}^{2} & \\
& +36 \frac{\Delta t^{2}}{p^{2} \Delta x \Delta y}\left[\left(\left|\alpha_{1}\right|+\left|\alpha_{2}\right|\right)^{2}+\frac{4}{\Delta x \Delta y}\left(\frac{1}{\Delta x^{2}}+\frac{1}{\Delta y^{2}}\right)\left\|\mathbf{u}^{r / p}\right\|_{h}^{2}\right]\left\|\mathbf{u}^{r / p}\right\|_{h}^{2}\left|\mathbf{u}^{r / p}\right|_{1, h}^{2} .
\end{aligned}
$$

For $r=0$ using 4.31, we get

$$
\left\|\mathbf{u}^{1 / p}\right\|_{h}^{2}+\frac{\Delta t}{2} \varepsilon^{2} \delta^{2} \eta^{2}\left|\mathbf{u}^{0}\right|_{1, h}^{2} \leq \exp \left(\frac{\Delta t}{p \varepsilon^{2}}\right)\left\|\mathbf{u}^{0}\right\|_{h}^{2} .
$$

Let us assume that (4.41) holds up to $r-1$. From the assumption for $s=1,2, \ldots, r-1$, we have

$$
\left\|\mathbf{u}^{s / p}\right\|_{h}^{2} \leq\left\|\mathbf{u}^{(s-1) / p}\right\|_{h}^{2}
$$

and

$$
\left\|\mathbf{u}^{s / p}\right\|_{h}^{2} \leq \exp \left(\frac{s \Delta t}{p \varepsilon^{2}}\right)\left\|\mathbf{u}^{0}\right\|_{h}^{2}
$$

The relation 4.42 becomes

$$
\begin{aligned}
\left\|\mathbf{u}^{(r+1) / p}\right\|_{h}^{2} & +\frac{\Delta t}{p} \varepsilon^{2} \delta^{2} \eta^{2}\left|\mathbf{u}^{r / p}\right|_{1, h}^{2} \leq \exp \left(\frac{\Delta t}{p \varepsilon^{2}}\right)\left\|\mathbf{u}^{r / p}\right\|_{h}^{2} \\
& +36 \frac{\Delta t^{2}}{p^{2} \Delta x \Delta y} \exp \left(\frac{2 r \Delta t}{p \varepsilon^{2}}\right)\left[\left(\left|\alpha_{1}\right|+\left|\alpha_{2}\right|\right)^{2}+\frac{4}{\Delta x \Delta y}\left(\frac{1}{\Delta x^{2}}+\frac{1}{\Delta y^{2}}\right)\left\|\mathbf{u}^{0}\right\|_{h}^{2}\right]\left\|\mathbf{u}^{0}\right\|_{h}^{2}\left|\mathbf{u}^{r / p}\right|_{1, h}^{2} \\
& \leq \exp \left(\frac{\Delta t}{p \varepsilon^{2}}\right)\left\|\mathbf{u}^{r / p}\right\|_{h}^{2}+\frac{\Delta t}{2 p} \varepsilon^{2} \delta^{2} \eta^{2}\left|\mathbf{u}^{r / p}\right|_{1, h}^{2}
\end{aligned}
$$


which shows us that 4.39 is true for $n=0$. From 4.44, we have

$$
\left\|\mathbf{u}^{1}\right\|_{h}^{2}+\frac{\Delta t}{p} \varepsilon^{2} \delta^{2} \eta^{2} \sum_{r=0}^{p-1} \exp \left(\frac{(p-1-r) \Delta t}{p \varepsilon^{2}}\right)\left|\mathbf{u}^{r / p}\right|_{1, h}^{2} \leq \exp \left(\frac{\Delta t}{\varepsilon^{2}}\right)\left\|\mathbf{u}^{0}\right\|_{h}^{2},
$$

which implies

$$
\left\|\mathbf{u}^{1}\right\|_{h}^{2} \leq \exp \left(\frac{\Delta t}{\varepsilon^{2}}\right)\left\|\mathbf{u}^{0}\right\|_{h}^{2}
$$

We then show 4.40 by using induction on $s$ for $n=0$. From the definition of $\mathbf{U}$, we have

$$
\left\|\mathbf{U}^{n}\right\|_{3 h}^{2} \leq\left\|\mathbf{u}^{n}\right\|_{h}^{2} .
$$

For $s=1$, from 4.29, we have

$$
\begin{aligned}
\left\|\mathbf{U}^{2}\right\|_{3 h}^{2} & -\exp \left(\frac{\Delta t}{\varepsilon^{2}}\right)\left\|\mathbf{U}^{1}\right\|_{3 h}^{2}+\Delta t \varepsilon^{2} \delta^{2} \eta^{2}\left|\mathbf{U}^{1}\right|_{1,3 h}^{2} \\
& -\frac{4 \Delta t^{2}}{\Delta x \Delta y}\left[\left(\left|\alpha_{1}\right|+\left|\alpha_{2}\right|\right)^{2}+\frac{4}{81 \Delta x \Delta y}\left(\frac{1}{\Delta x^{2}}+\frac{1}{\Delta y^{2}}\right)\left\|\mathbf{U}^{1}\right\|_{3 h}^{2}\right]\left\|\mathbf{U}^{1}\right\|_{3 h}^{2}\left|\mathbf{U}^{1}\right|_{1,3 h}^{2} \leq 0 .
\end{aligned}
$$

Then using 4.45 and 4.46, we have

$$
\begin{aligned}
\left\|\mathbf{U}^{2}\right\|_{3 h}^{2} & -\exp \left(\frac{\Delta t}{\varepsilon^{2}}\right)\left\|\mathbf{U}^{1}\right\|_{3 h}^{2}+\Delta t \varepsilon^{2} \delta^{2} \eta^{2}\left|\mathbf{U}^{1}\right|_{1,3 h}^{2} \\
& -\frac{4 \Delta t^{2}}{\Delta x \Delta y} \exp \left(\frac{2 \Delta t}{\varepsilon^{2}}\right)\left[\left(\left|\alpha_{1}\right|+\left|\alpha_{2}\right|\right)^{2}+\frac{4}{81 \Delta x \Delta y}\left(\frac{1}{\Delta x^{2}}+\frac{1}{\Delta y^{2}}\right)\left\|\mathbf{u}^{0}\right\|_{h}^{2}\right]\left\|\mathbf{u}^{0}\right\|_{h}^{2}\left|\mathbf{U}^{1}\right|_{1,3 h}^{2} \leq 0,
\end{aligned}
$$

and using (4.32), we arrive at

$$
\left\|\mathbf{U}^{2}\right\|_{3 h}^{2}+\frac{\Delta t}{2} \varepsilon^{2} \delta^{2} \eta^{2}\left|\mathbf{U}^{1}\right|_{1,3 h}^{2} \leq \exp \left(\frac{\Delta t}{\varepsilon^{2}}\right)\left\|\mathbf{U}^{1}\right\|_{3 h}^{2} .
$$

We now assume that 4.40 holds true up to the order $q-1$, that is

$$
\left\|\mathbf{U}^{q}\right\|_{3 h}^{2}+\frac{\Delta t}{2} \varepsilon^{2} \delta^{2} \eta^{2}\left|\mathbf{U}^{q-1}\right|_{1,3 h}^{2} \leq \exp \left(\frac{\Delta t}{\varepsilon^{2}}\right)\left\|\mathbf{U}^{q-1}\right\|_{3 h}^{2} .
$$

and we observe that

$$
\left\|\mathbf{U}^{s+1}\right\|_{3 h}^{2} \leq \exp \left(\frac{\Delta t}{\varepsilon^{2}}\right)\left\|\mathbf{U}^{s}\right\|_{3 h}^{2}, \text { for } s=1, \ldots, q-1 .
$$

From 4.38 and 4.49) together with 4.32 we obtain the result. Thus using 4.1 and 4.6), we find

$$
\left\|\mathbf{u}^{s+1}\right\|_{h}^{2} \leq \exp \left(\frac{\Delta t}{\varepsilon^{2}}\right)\left\|\mathbf{u}^{s}\right\|_{h}^{2}, \text { for } s=0, \ldots, q .
$$

Now suppose that 4.39 and 4.40 holds up to the order $n$. Using the same approach as in the case $n=0$, it can be easily proved by induction on $r$ and $s$. Hence, 4.39) and 4.40 hold for any $n=z(q+1)$, where $z \in \mathbb{N}_{+}$.

Therefore, the proof is complete. 
Remark 4.1. By the subscript 3h, we mean the discrete operators, discrete norms and semi-norms are applied on the coarser discretization.

Remark 4.2. To compare the stability regions of the explicit finite volume methods, we use $\frac{\Delta t}{p}$ on the fine mesh and $\Delta t$ on the coarser mesh as discussed in this section.

- When $p \leq 9$, the multilevel method has the same region of stability as the one-level method on the fine mesh but smaller region of stability than the one-level method on the coarse mesh.

- When $p \geq 81$, the multilevel method has the same region of stability as the one-level method on the coarse mesh but smaller region of stability than the one-level method on the fine mesh.

- When $9<p<81$, the multilevel method is less restrictive than the one-level method on the fine mesh and more restrictive than the one-level method on the coarse mesh.

\section{$5 \quad$ Numerical Simulations}

In this section, some numerical simulations of the 2D convective Cahn-Hilliard equation, 1.1), with specified initial condition and periodic boundary conditions at some values of $T$ are presented. All the results are computed in a matlab platform using Windows 10 Intel CORE i3, 6G RAM PC and the parameters are chosen as: $\alpha_{1}=\alpha_{2}=\frac{1}{6}, p=5$ and $q=8$.

For the one-level finite volume methods, we use the following temporal and spatial step sizes

- One-level method on the fine mesh (Fine): time step $\Delta t / p$ and spatial step sizes $\Delta x=\Delta y$.

- One-level method on the coarse mesh (Coarse): time step size $\Delta t$ and spatial step sizes $3 \Delta x=3 \Delta y$.

For the implicit one-level method, $\tilde{\mathbf{u}}^{n}$ is given by the relation:

$$
\tilde{\mathbf{u}}^{n}=\frac{1}{2}\left(\mathbf{u}^{n}+\mathbf{u}^{n-1}\right), \text { for } n=1,2, \ldots, M-1 .
$$

Similarly for the implicit multilevel method, for a non-negative integer $m$ and $n=m(q+1)$, we use the following approximations:

$$
\begin{aligned}
\tilde{\mathbf{u}}^{m(q+1)+r / p} & =\frac{1}{2}\left(\mathbf{u}^{m(q+1)+r / p}+\mathbf{u}^{m(q+1)+(r-1) / p}\right), \text { for } r=1, \ldots, p-1, \\
\tilde{\mathbf{u}}^{m(q+1)} & =\mathbf{u}^{m(q+1)}, \\
\tilde{\mathbf{U}}^{m(q+1)+s} & =\frac{1}{2}\left(\mathbf{U}^{m(q+1)+s}+\mathbf{U}^{m(q+1)+s-1}\right), \text { for } s=1, \ldots, q,
\end{aligned}
$$

and for both implicit methods $\tilde{\mathbf{u}}^{0}=\mathbf{u}^{0}$.

To test the numerical methods, we consider the exact solution

$$
u(x, y, t)=\sin \left(\frac{2 \pi x}{L}\right) \sin \left(\frac{2 \pi y}{L}\right) \cos (2 \pi t),
$$

where $L_{1}=L_{2}=L=3$, from which the source term is obtained on substitution of (1.1). As shown by Fig. 2, it is observed that the numerical results obtained using the multilevel finite volume methods are close to the results obtained from one-level methods on the fine mesh as compared to the one-level on the coarse mesh. There is no need to plot $u$ versus $y$ because of the similarities with $u$ versus $x$.

Tables 1. 2 show that we can save more time using the multilevel method as compared to the one-level methods on the fine mesh. From the numerical simulations, it is observed that all methods are second order accurate in space and the solutions obtained from the multilevel methods are intermediate between the ones obtained from one-level methods on the fine mesh and on the coarse mesh. 


\begin{tabular}{|c|c|c|c|c|c|}
\hline Method & $\Delta x(=\Delta y)$ & $\Delta t$ & $L_{2}$-error & CPU time & $L_{2}$ Rate \\
\hline \multirow{3}{*}{ Fine } & 0.2 & 0.01 & 0.0518 & 1.032 & \\
\cline { 2 - 6 } & 0.1 & 0.0025 & 0.0131 & 15.134 & 1.9594 \\
\cline { 2 - 6 } & 0.05 & 0.000625 & 0.0033 & 1232.888 & 2 \\
\hline \multirow{4}{*}{ Coarse } & 0.2 & 0.01 & 0.3947 & 0.150 & \\
\cline { 2 - 6 } & 0.1 & 0.0025 & 0.1128 & 0.272 & 1.5661 \\
\cline { 2 - 6 } & 0.05 & 0.000625 & 0.0291 & 3.822 & 1.8806 \\
\cline { 2 - 6 } & 0.025 & 0.00015625 & 0.0073 & 159.934 & 1.9607 \\
\hline \multirow{4}{*}{ Multilevel } & 0.2 & 0.01 & 0.0518 & 1.341 & \\
\cline { 2 - 6 } & 0.1 & 0.0025 & 0.0279 & 4.643 & 0.8927 \\
\cline { 2 - 6 } & 0.05 & 0.000625 & 0.0098 & 271.597 & 1.5094 \\
\cline { 2 - 6 } & 0.025 & 0.00015625 & 0.0027 & 15701.626 & 1.8598 \\
\hline
\end{tabular}

Table 1: Convergence rate, CPU time and $L_{2}$-error for some values of spatial step sizes and $\Delta t$ for the implicit methods at $T=0.01$.

\begin{tabular}{|c|c|c|c|c|c|}
\hline Method & $\Delta x(=\Delta y)$ & $\Delta t$ & $L_{2}$-error & CPU time & $L_{2}$ Rate \\
\hline \multirow{3}{*}{ Fine } & 0.2 & 0.0002 & 0.0441 & 1.765 & \\
\cline { 2 - 6 } & 0.1 & 0.0000125 & 0.0112 & 73.639 & 1.9773 \\
\cline { 2 - 6 } & 0.05 & 0.00000078125 & 0.0031 & 10267.945 & 1.8532 \\
\hline \multirow{3}{*}{ Coarse } & 0.2 & 0.0002 & 0.3749 & 0.571 & \\
\cline { 2 - 6 } & 0.1 & 0.0000125 & 0.0983 & 2.079 & 1.9312 \\
\cline { 2 - 6 } & 0.05 & 0.00000078125 & 0.0249 & 117.542 & 1.9810 \\
\hline \multirow{4}{*}{ Multilevel } & 0.2 & 0.0002 & 0.0449 & 0.469 & \\
\cline { 2 - 6 } & 0.1 & 0.0000125 & 0.0143 & 10.725 & 1.6507 \\
\cline { 2 - 6 } & 0.05 & 0.00000078125 & 0.0043 & 723.252 & 1.7336 \\
\hline
\end{tabular}

Table 2: Convergence rate, CPU time and $L_{2}$-error for some values of spatial step sizes and $\Delta t$ for the explicit methods at $T=0.001$. 


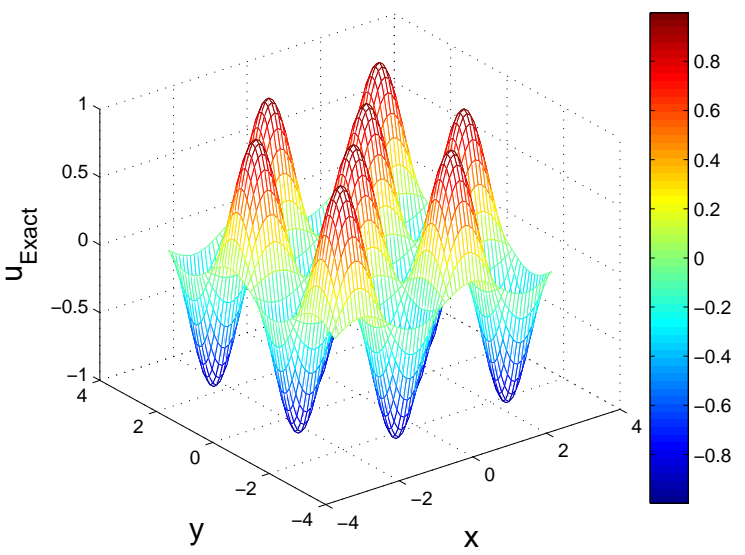

(a) Exact when $\Delta x=\Delta y=0.1$ and $\Delta t=0.0025$ at $T=$ 0.01 .

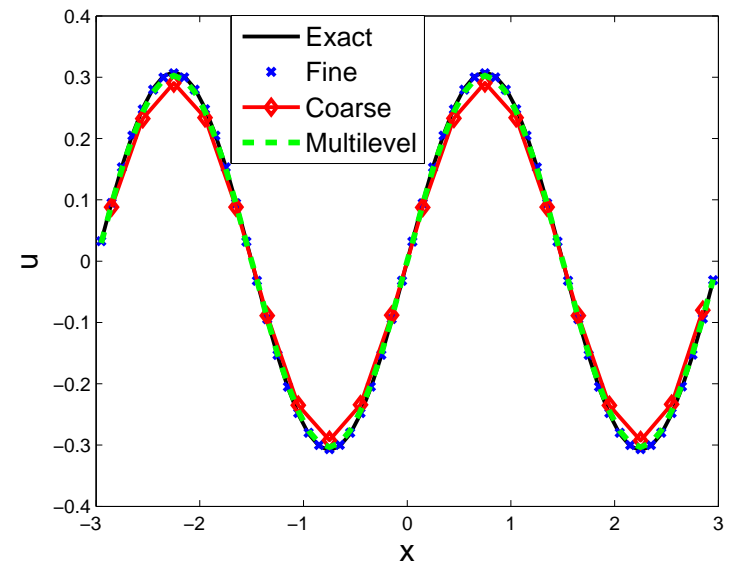

(c) $u$ versus $x$ obtained from implicit methods when $\Delta x=$ $\Delta y=0.1, \Delta t=0.0025$ at the cells with centre $y=0.15$ and $T=0.01$.

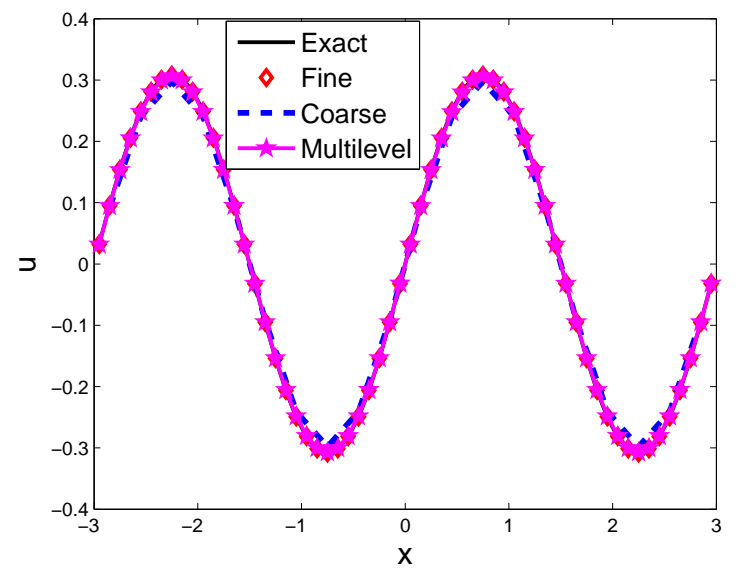

(e) $u$ versus $x$ obtained from explicit methods when $\Delta x=$ $\Delta y=0.1, \Delta t=0.0000125$ at the cells with centre $y=0.15$ and $T=0.001$.

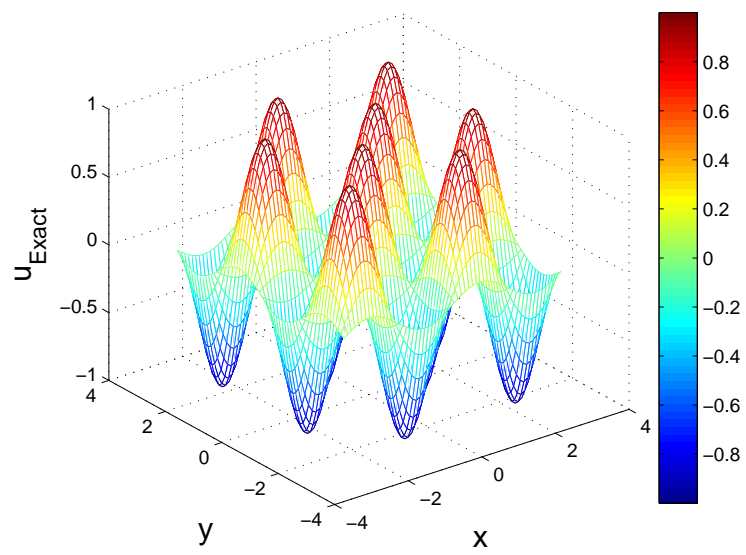

(b) Exact when $\Delta x=\Delta y=0.1$ and $\Delta t=0.0000125$ at $T=0.001$.

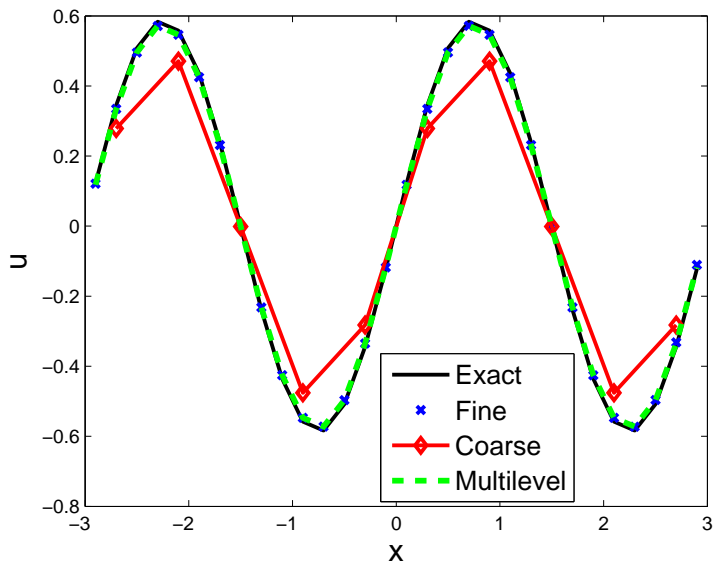

(d) $u$ versus $x$ obtained from implicit methods when $\Delta x=$ $\Delta y=0.2, \Delta t=0.01$ at the cells with centre $y=0.3$ and $T=0.01$.

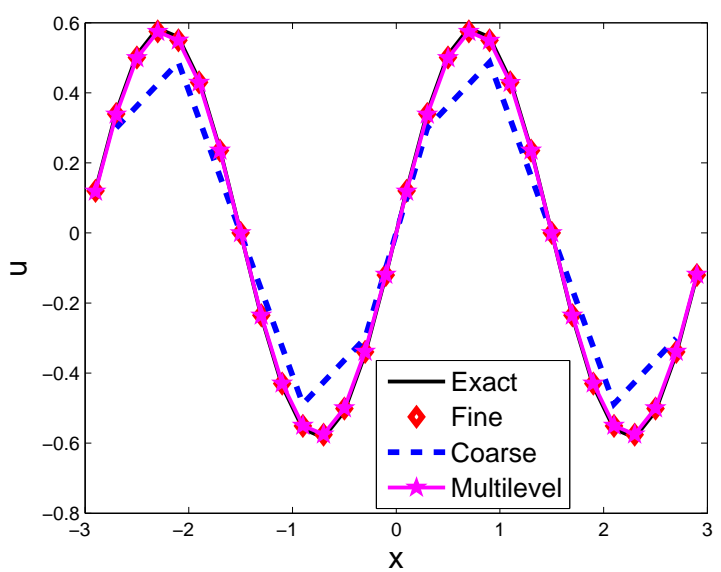

(f) $u$ versus $x$ obtained from explicit methods when $\Delta x=$ $\Delta y=0.2, \Delta t=0.0002$ at the cells with centre $y=0.3$ and $T=0.001$.

Figure 2: Numerical results for some values of spatial step sizes and $\Delta t$. 


\section{Conclusion}

We have extended the work of [22, 23] in two directions: first, we have considered a nonlinear equation in which the nonlinear term has been linearized following Mickens' rules. Secondly, we have shown that the method can be adapted to higher order partial differential equations. In this paper, four numerical methods have been presented and analyzed. The implicit methods discussed here are linear and easy to implement. Existence, uniqueness of solutions for the schemes formulated are discussed and detailed convergence analysis of implicit schemes is furnished. We compare the multilevel methods with the one-level methods by means of stability, convergence and CPU time. It is shown that the multilevel methods are faster than the one-level methods on the fine mesh. We also study the stability of these schemes which allow us to make a classification based on region of stability. But as the numerical experiments reveal, comparing these schemes only with the stability is misleading, hence the CPU time is good indicator for a classification. From the convergence analysis, it is proven that all the methods are second order accurate in space and it is validated by numerical experiments. Our future plan is to extend this work to Navier Stokes equations and its variants.

\section{Acknowledgments}

A.R. Appadu is grateful to the South African DST/NRF SARChI Chair on Mathematical Models and Methods in Bioengineering and Biosciences of the University of Pretoria and to the National Research Foundation of South African Grant Number 95864. J.K. Djoko is funded through the incentive fund N00 401 Project 85796. H.H. Gidey is grateful to the University of Pretoria, African Institute for Mathematical Sciences (AIMS)-South Africa, DST-NRF Centre of Excellence in Mathematical and Statistical Sciences (CoE-MaSS) and Aksum University (Ethiopia) for their financial support for his PhD studies.

We thank the referee for many remarks that have led to some improvements in the text.

\section{References}

[1] R. E. Mickens, Nonstandard Finite Difference Models of Differential Equations, World Scientific, Singapore, 1994.

[2] K. Leung, Theory of morphological instability in driven systems, Statistical Physics 61 (1/2) (1990) $345-364$.

[3] C. L. Emmott, A. J. Bray, Coarsening dynamics of a one-dimensional driven Cahn-Hilliard system, Physical Review E 54 (5) (1996) 4568-4575.

[4] C. Yeung, T. Rogers, A. Hernandez-Machado, D. Jasnow, Phase separation dynamics in driven diffusive systems, Statistical Physics 66 (1992) 1245-1250.

[5] A. A. Golovin, S. H. Davis, A. A. Nepomnyashcy, A convective Cahn-Hilliard model for the formation of facets and corners in crsytal growth, Physica D 122 (1998) 202-230.

[6] A. A. Golovin, S. H. Davis, A. A. Nepomnyashcy, Modeling the formation of facets and corners using a convective Cahn-Hilliard equation, Journal of Crystal Growth 198/199 (1999) 1245-1250.

[7] W. Cahn, Phase separation by spinodal decomposition in isotropic systems, Journal of Chemical Physics 42 (1965) 93-99.

[8] A. J. Bray, Theory of phase-ordering kinetics, Advances in Physics 43 (3) (1994) 357-459.

[9] C. M. Elliott, Z. Songmu, On the Cahn-Hilliard equation, Archive for Rational Mechanics and Analysis 96 (1986) 339-357. 
[10] N. Khiari, T. Achouri, M. L. B. Mohamed, K. Omrani, Finite difference approximations for the CahnHilliard equations, Numerical methods for partial differential equations 23 (2007) 437-455.

[11] H. Song, Energy stable and large time-stepping methods for the Cahn-Hilliard equation, International Journal of Computer Mathematics 92 (10) (2015) 2091-2108.

[12] A. A. Golovin, A. A. Nepomnyashchy, S. H. Davis, M. A. Zaks, Convective Cahn-Hilliard models: From coarsening to roughening, Physical Review Letters 86 (8) (2001) 1550-1553.

[13] S. J. Watson, F. Otto, B. Y. Rubinstein, S. H. Davis, Coarsening dynamics of the convective CahnHilliard equation, Physica D 178 (2003) 127-148.

[14] A. Podolny, M. A. Zaks, B. Y. Rubinstein, A. A. Golovin, A. A. Nepomnyashchy, Dynamics of domin walls governed by the convective Cahn-Hilliard equation, Physica D 201 (2005) 291-305.

[15] M. A. Zaks, A. Podolny, A. A. Nepomnyashchy, A. A. Golovin, Periodic stationary patterns governed by a convective Cahn-Hilliard equation, SIAM Journal of Applied Mathematics 66 (2) (2006) 700-720.

[16] A. Eden, V. K. Kalantarov, The convective Cahn-Hilliard equation, Applied Mathematics Letters 20 (2007) 455-461.

[17] X. Zhao, C. Liu, Optimal control of the convective Cahn-Hilliard equation, Applicable Analysis 92 (5) (2013) 1028-1045.

[18] A. A. Aderogba, M. Chapwanya, J. K. Djoko, On fractional step-splitting scheme for the Cahn-Hilliard equation, Engineering Computations 31 (2014) 1151-1168.

[19] A. Eden, V. K. Kalantarov, 3D convective Cahn-Hilliard equation, Communications on Pure and Applied Analysis 6 (4) (2007) 1075-1086.

[20] X. Zhao, C. Liu, Optimal control of the convective Cahn-Hilliard equation in 2D case, Applied Mathematcics and Optimization 70 (2014) 61-82.

[21] R. Temam, Infinite-dimensional dynamical systems in mechanics and physics, Vol. 68, Springer Science \& Business Media, 2012.

[22] A. Bousquet, M. Marion, M. Pectu, R. Temam, Multilevel finite volume methods and boundary value conditions for geophysical flows, Computers and Fluids 74 (2013) 66-90.

[23] A. Bousquet, M. Marion, R. Temam, Finite volume multilevel approximation of the shallow water equations with a time explicit scheme, International Journal of Numerical Analysis and Modeling 11 (4) (2014) $762-786$.

[24] Y. He, K. Liu, A multilevel finite element method in space-time for the Navier-Stokes problem, Wiley InterScience (2005) 1052-1078.

[25] S. Faure, J. Laminie, R. Temam, Finite volume discretization and multilevel methods in flow problems, Scientific Computing $25(1 / 2)$ (2005) 231-261.

[26] A. Bousquet, R. Temam, A finite volume multilevel approximation of the shallow-water equations, in: J. C. F. Pereira, A. Sequeira (Eds.), European Conference on Computational Fluid Dynamics ECCOMAS CFD 2010, Vol. V, 14-17 June 2010.

[27] K. Adamy, A. Bousquet, S. Faure, J. Lamine, R. Temam, A multilevel method for finite volume discretization of the two-dimensional nonlinear shallow-water equations, Ocean Modeling 33 (2010) 235256 . 
[28] R. Anguelov, J. M.-S. Lubuma, Contributions to the mathematics of the nonstandard finite difference method and applications, Numerical Methods for Partial Differential Equations 17 (5) (2001) 518-543.

[29] J. K. Djoko, On the long-time stability of a backward euler scheme for Burgers' equation with polynomial force, Numerical Methods for Partial Differential Equations 24 (2008) 1371-1387.

[30] M. S. Gockenbach, Finite-Dimensional Linear Algebra, CRC Press, Taylor and Francis Group, 2010.

[31] R. Temam, Navier-Stokes Equations: Theory and Numerical Analysis, Vol. 2, North-Holland, 1979.

\section{Appendix A Taylor's expansion about $v_{i, j}^{n}$}

In this section, we prove the Taylor's expansion given by (3.17), (3.18) and (3.19). Note for simplicity that we omit $\left.\right|_{i, j} ^{n}$ on the expanded terms (right hand sides of each equations).

Proof of (3.17).

To find the Taylor's expansion of the approximation of the fourth order derivative, we use the relation

$$
\Delta_{h}^{2} v_{i, j}^{n+1}=\Delta_{1, h}^{2} v_{i, j}^{n+1}+\Delta_{2, h} \Delta_{1, h} v_{i, j}^{n+1}+\Delta_{1, h} \Delta_{2, h} v_{i, j}^{n+1}+\Delta_{2, h}^{2} v_{i, j}^{n+1} .
$$

It is clear that

$$
\begin{aligned}
& \Delta_{1, h}^{2} v_{i, j}^{n+1}=u_{x x x x}+\mathcal{O}\left(\Delta t+\Delta x^{2}\right) . \\
& \Delta_{2, h}^{2} v_{i, j}^{n+1}=u_{\text {yyy }}+\mathcal{O}\left(\Delta t+\Delta y^{2}\right) .
\end{aligned}
$$

We only find the Taylor's expansion of the second term of the right hand side of (A.1) and hence the expansion of the third term can be obtained accordingly. Using central difference approximation, we have

$$
\Delta_{2, h} \Delta_{1, h} v_{i, j}^{n+1}=\frac{\left(u_{i+1, j+1}^{n+1}-2 u_{i, j+1}^{n+1}+u_{i-1, j+1}^{n+1}\right)-2\left(u_{i+1, j}^{n+1}-2 u_{i, j}^{n+1}+u_{i-1, j}^{n+1}\right)+\left(u_{i+1, j-1}^{n+1}-2 u_{i, j-1}^{n+1}+u_{i-1, j-1}^{n+1}\right)}{\Delta x^{2} \Delta y^{2}} .
$$

Then applying Taylor's expansion, one can verify that

$$
\Delta_{2, h} \Delta_{1, h} v_{i, j}^{n+1}=u_{x x y y}+\mathcal{O}\left(\Delta t+\Delta x^{2}+\Delta x \Delta y+\Delta y^{2}\right)
$$

and

$$
\Delta_{1, h} \Delta_{2, h} v_{i, j}^{n+1}=u_{y y x x}+\mathcal{O}\left(\Delta t+\Delta x^{2}+\Delta x \Delta y+\Delta y^{2}\right) .
$$

Therefore, we conclude (3.17).

Proof of 3.18.

$\nabla_{1, h}^{+}\left(\psi_{i-\frac{1}{2}, j}^{n} \nabla_{1, h}^{-} v_{i, j}^{n+1}\right)=\frac{3}{2 \Delta x}\left(\left(v_{i+1, j}^{n}\right)^{2}-\left(v_{i-1, j}^{n}\right)^{2}\right) \frac{\left(v_{i+1, j}^{n+1}-v_{i, j}^{n+1}\right)}{\Delta x}+\frac{3}{2}\left(\left(v_{i, j}^{n}\right)^{2}+\left(v_{i-1, j}^{n}\right)^{2}\right) \Delta_{1, h} v_{i, j}^{n+1}-\Delta_{1, h} v_{i, j}^{n+1}$.

Clearly $\Delta_{1, h} v_{i, j}^{n+1}=u_{x x}+\mathcal{O}\left(\Delta t+\Delta x^{2}\right)$. Making use of Taylor's expansion, we obtain

$$
\frac{3}{2 \Delta x^{2}}\left(\left(v_{i+1, j}^{n}\right)^{2}-\left(v_{i-1, j}^{n}\right)^{2}\right)\left(v_{i+1, j}^{n+1}-v_{i, j}^{n+1}\right)=6 u u_{x}^{2}+3 \Delta x u u_{x} u_{x x}+\mathcal{O}\left(\Delta t+\Delta x^{2}\right),
$$


and

$$
\frac{3}{2}\left(\left(v_{i, j}^{n}\right)^{2}+\left(v_{i-1, j}^{n}\right)^{2}\right) \Delta_{1, h} v_{i, j}^{n+1}=3 u^{2} u_{x x}-3 u u_{x} u_{x x}+\mathcal{O}\left(\Delta t+\Delta x^{2}\right),
$$

which give

$$
\nabla_{1, h}^{+}\left(\psi_{i-\frac{1}{2}, j}^{n} \nabla_{1, h}^{-} v_{i, j}^{n+1}\right)=6 u u_{x}^{2}+3 u^{2} u_{x x}-u_{x x}++\mathcal{O}\left(\Delta t+\Delta x^{2}\right) .
$$

In a similar way, we obtain

$$
\nabla_{2, h}^{+}\left(\psi_{i, j-\frac{1}{2}}^{n} \nabla_{2, h}^{-} v_{i, j}^{n+1}\right)=6 u u_{y}^{2}+3 u^{2} u_{y y}-u_{y y}++\mathcal{O}\left(\Delta t+\Delta y^{2}\right) .
$$

Combining A.6 and (A.7), we obtain (3.18).

Proof of (3.19).

We recall that

$$
\begin{aligned}
C_{h}\left(\boldsymbol{v}^{n+1}, \tilde{\boldsymbol{v}}^{n}\right)_{i, j}= & \left.\frac{\alpha_{1}}{\Delta x}\left[v_{i+1, j}^{n+1} \tilde{v}_{i+1, j}^{n}-v_{i-1, j}^{n+1} \tilde{v}_{i, j}^{n}\right]\right]+\frac{\alpha_{2}}{\Delta x}\left[v_{i+1, j}^{n+1} \tilde{v}_{i, j}^{n}-v_{i-1, j}^{n+1} \tilde{v}_{i-1, j}^{n}\right] \\
& +\frac{\alpha_{1}}{\Delta y}\left[v_{i, j+1}^{n+1} \tilde{v}_{i, j+1}^{n}-v_{i, j-1}^{n+1} \tilde{v}_{i, j}^{n}+\frac{\alpha_{2}}{\Delta y}\left[v_{i, j+1}^{n+1} \tilde{v}_{i, j}^{n}-v_{i, j-1}^{n+1} \tilde{v}_{i, j-1}^{n}\right] .\right.
\end{aligned}
$$

Then applying Taylor's expansion, we obtain

$$
\frac{v_{i+1, j}^{n+1} \tilde{v}_{i+1, j}^{n}-v_{i-1, j}^{n+1} \tilde{v}_{i, j}^{n}}{\Delta x}=c_{0}\left[3 u u_{x}+\frac{\Delta x}{2} u u_{x x}+\Delta x u_{x}^{2}\right]+\mathcal{O}\left(\Delta t+\Delta x^{2}\right),
$$

and

$$
\frac{v_{i+1, j}^{n+1} \tilde{v}_{i, j}^{n}-v_{i-1, j}^{n+1} \tilde{v}_{i-1, j}^{n}}{\Delta x}=c_{0}\left[3 u u_{x}-\frac{\Delta x}{2} u u_{x x}-\Delta x u_{x}^{2}\right]+\mathcal{O}\left(\Delta t+\Delta x^{2}\right),
$$

where

$$
c_{0}=a_{1}+a_{2}+\cdots+a_{m_{0}} .
$$

Hence

$$
\begin{array}{r}
\frac{\alpha_{1}}{\Delta x}\left[v_{i+1, j}^{n+1} \tilde{v}_{i+1, j}^{n}-v_{i-1, j}^{n+1} \tilde{v}_{i, j}^{n}\right]+\frac{\alpha_{2}}{\Delta x}\left[v_{i+1, j}^{n+1} \tilde{v}_{i, j}^{n}-v_{i-1, j}^{n+1} \tilde{v}_{i-1, j}^{n}\right]=3 c_{0}\left(\alpha_{1}+\alpha_{2}\right) u u_{x} \\
+\frac{\Delta x c_{0}}{2}\left(\alpha_{1}-\alpha_{2}\right) u u_{x x}+\Delta x c_{0}\left(\alpha_{1}-\alpha_{2}\right) u_{x}^{2}+\mathcal{O}\left(\Delta t+\Delta x^{2}\right) .
\end{array}
$$

In a similar way, one obtains

$$
\begin{array}{r}
\frac{\alpha_{1}}{\Delta y}\left[v_{i, j+1}^{n+1} \tilde{v}_{i, j+1}^{n}-v_{i, j-1}^{n+1} \tilde{v}_{i, j}^{n}\right]+\frac{\alpha_{2}}{\Delta y}\left[v_{i, j+1}^{n+1} \tilde{v}_{i, j}^{n}-v_{i, j-1}^{n+1} \tilde{v}_{i, j-1}^{n}\right]=3 c_{0}\left(\alpha_{1}+\alpha_{2}\right) u u_{y} \\
+\frac{\Delta y c_{0}}{2}\left(\alpha_{1}-\alpha_{2}\right) u u_{y y}+\Delta y c_{0}\left(\alpha_{1}-\alpha_{2}\right) u_{y}^{2}+\mathcal{O}\left(\Delta t+\Delta y^{2}\right) .
\end{array}
$$

Combining A.9 and A.10, we obtain (3.19). 